\title{
HARMONIC EXPANSIONS OF FUNCTIONS OF TWO YARIABLES
}

\author{
By A. C. Dixon.
}

\begin{abstract}
[Received September 9th, 1906.-Read November Sth, 1906.- Received, in revised form, June 30th, 1907.]
\end{abstract}

THE object of this paper is to extend to functions of two variables the results of an earlier one* (Proc. London Math. Soc., Ser. 2, Vol. 3, pp. 83-103), and so to establish the validity of such expansions in series of partial harmonics as have been proposed by Klein and Bocher. ${ }^{+}$

Each of two real variables $x$, $y$ has a range from 0 to 1 , and a fairly general function of them is expanded in a double series, each term of which is the product of a function of $x$ and a function of $y$; these functions are respectively determined by differential equations, in which occur two parameters $\lambda, \mu$, which have different values in the different terms of the series.

By means of Poincaré's extension of Cauchy's residue theorem au expression is given for the sum of a finite number of terms of the series as a multiple integral: the choice of a suitable field for the integration is a matter of some difficulty. The convergency is investigated by a discussion of the order of magnitude of the remainder, in which certain approximate solutions of the differential equations are used. This discussion is somewhat intricate, owing to difficulties which do not present themselves in the simpler case of functions of one variable.

The question of the expansibility of a given function in a series of multiples of given functions divides itself into two parts, one relating to the nature of the function to be expanded, and the other to what may be called the completeness of the proposed series. For instance, it is impossible to expand $\sin x(0<x<\pi)$ in multiples of $\sin 2 x$, $\sin 3 x$, $\sin 4 x, \ldots$, because this series of functions is incomplete; but eren the complete series $\sin x, \sin 2 x, \ldots$ is inadequate to express a function which

* Hereinafter referred to as I. There are important papers un the same subject by Stekloff in the Conptes Rendlus for 1902, pp. \$\$8-8.51, and Hilbert (Gütt. Sichrichten, 1904).

+ For references see $\$ 32$ (note) below. 
is not a limit of continuous functions. It follows from the result of this paper that the double series discussed are equivalent in this kind of completeness to double Fourier series; it is, in fact, possible to expand in a series of the present kind the general term of a double Fourier series, and conversely.

The following is a list of the headings of paragraphs, and under each heading are indicated the symbols introduced in the corresponding paragraph and used afterwards :-

1. The Fundamental Differential Equations. $(x, y, \lambda, \mu, s, T, U, V, X, Y, \phi, \psi$.

2. $\phi$ and its Derivatives. $\left[\phi(x, a), \phi^{\prime}, \phi^{\prime}, \phi^{\prime \prime}, \|^{\prime}\right.$.)

3. Functions $\phi$ with different Parameters.

4. Reality of Solutions of $\Theta=0, Z=0 . \quad\left(\Theta, Z, E, E_{1}, \ldots, G, G_{1}, \ldots.\right)$

5. Distinctuess of Solutions of $\Theta=0, Z=0$. (H.)

6. A certain Double Integral. ( $\Phi, \Psi$.

6a. Known Results relating to Double Contour Integrals. $(L, \lambda, \Omega$.)

6b. General Idea of the Proposed Application of these Results.

7. Approximations by means of Hyperbolic Functions when $\lambda, \mu$ are Great. $\left(P, Q, f, \delta, d_{\rho}\right.$, $d \sigma, k, i \mid$, " normal.")

8. Approximation by means of Riccati Functions. ( $\omega, v 0$.

9. Approximate Value of $\omega$.

10. Approximations to $\omega(a, 0)$ and its Derivatives, when $a$ is Negative and Great. $(-A, a, B, \beta$.

11. The same when the Vectorial Angle of $a$ is between $\frac{1}{3} \pi$ and $\frac{5}{3} \pi .(\checkmark)$

12. The same for other Great Values of $a$. Values of $A B, a, \beta$.

13. The Function $x .(x \cdot)$

14. Approximation to $\omega(b, a)$.

15. Application to $\phi$.

16. Case when $P$ Vanishes near a Terrninus.

17. Case when $P$ Vanishes more than Once. ( $\Upsilon$.)

18. Cau the Terms in $\Sigma \Upsilon$ destroy each other? $\left(p, q, x_{1}, x_{2}, \ldots.\right)$

19. Another Method.

20, 21. Care of Terminal Short Sections. (s.)

22. Stationary Values of $S / T$. Riccati case. $(\xi, \eta, \zeta, \omega$.

23. Hyperbolic Case.

24. Combination of the Two Cases.

25. Short Sections.

26. Approximate Values of $\Theta, \Phi$. ( $\delta$.)

27. Distribution of the Values of $\mu$ for which $\Theta=0 . \quad\left(p_{1}, p_{2}, q_{1}, q_{2}, r_{1}, r_{2}, s_{1}, s_{2}, \tau, C, D\right.$, $C^{\prime}, D^{\prime}$.)

28-31. The Field of Integration $(k, p, q, g, h)\left(m_{0}, m_{1}, \ldots, \mu_{11}, \mu_{1}, \ldots, a, \beta, \gamma, g^{\prime}, h^{\prime}, \nu\right)(K)\left(g^{\prime}, Q\right)$.

32. Supplement to $\S 6 b . \quad\left(\lambda, s, \mu_{r s .}\right)$

33. Expansion of an Arbitrary Analytical Function. $\left(f, H, x_{11}, x_{1}, y_{11}, y_{1}.\right)$

34. The Remainder. A Lemua.

35-37. Discussion of the Remainder.

38. Statement of the Result.

39. Case when $\vec{E}_{1}$ or $G_{4}=0$.

40. Double Solutions of the Equatious $\Theta=0, Z=0$.

41. Form of the Gieneral Term in the Expansion.

42. Special Cases.

43. Validity at the Boundary. 


\section{The Fundamental Differential Equations.}

1. Let $x, y$ be two real variables, each of which may have any value from 0 to 1 , inclusive, and let $\lambda, \mu$ be parameters.

Let $\phi$ denote a function of $x$ and the parameters, satisfying the equation

$$
\frac{d^{2} \phi}{d x^{2}}+(\lambda S-\mu T+X) \phi=0,
$$

where $S, T, X$ are real analytical functions of $x$ only.

Let $\psi$ denote a function of $y$ and the parameters, satisfying the equation

$$
\frac{d^{2} \psi}{d y^{2}}+(-\lambda U+\mu V+Y) \psi=0
$$

where $U, V, Y$ are real analytical functions of $y$ only.

Suppose also that, for all values of $x, y$, the sign of $S V-U T$ is constant, say positive. By a simple transformation of $\lambda, \mu$ we may make $S, T, U, V$ all positive. For since $S V-U T$ is never zero, no value of $S / T$ can be equal to any value of $U / V$, and one of these two fractions, say $U / V$, must be always finite. Let $\alpha$ be a real value which neither of the fractions takes : then $S-\alpha T, U-\alpha V$ are of constant signs. If these signs are opposite, let $\alpha$ be changed and let it travel through all the values of $U / V$ to another value $\alpha^{\prime}$, which is never taken by either fraction; then $S-\alpha^{\prime} T, U-\alpha^{\prime} V$ will have the same constant sign. The values $a, a^{\prime}$ are only restricted by inequalities, and we may therefore take two values $\alpha, \beta$, such that

$$
S-\alpha^{\prime} T, S-\beta T, U-\alpha V, U-\beta V, \beta-\alpha
$$

have the same sign. Then

$$
\lambda S-\mu T=\lambda_{1} S_{1}-\mu_{1} T_{1}, \quad-\lambda U+\mu V=-\lambda_{1} U_{1}+\mu_{1} V_{1},
$$

where

$$
\begin{array}{cc}
S_{1}=(S-\beta T) /(\beta-\alpha), & T_{1}=(S-\alpha T) /(\beta-\alpha), \\
U_{1}=(U-\beta V) /(\beta-\alpha), & V_{1}=(U-\alpha V) /(\beta-\alpha), \\
\lambda_{1}=\mu-\alpha \lambda, & \mu_{1}=\mu-\beta \lambda, \\
S_{1} V_{1}-U_{1} T_{1}=(S V-U T) /(\beta-\alpha) .
\end{array}
$$

Hence, if $\lambda_{1}, \mu_{1}$ are used as parameters instead of $\lambda, \mu$ the signs will be positive as desired unless $\beta-\alpha$ is negative; if $\beta-\alpha$ is negative we may take $-\mu_{1},-\lambda_{1}$ as parameters.

It will be supposed that this transformation has been made, so thit $S, T, U, V, S V-U T$ are all positive, and never zero. 
$\phi$ and its Derivatives.

2. The notation $\phi(x, a)$ will be used for a function $\phi$ satisfying the equation (1), and also the initial conditions $\phi=0, d \phi / d x=1$, when $x=a, \phi^{\prime}(x, a), \phi^{\prime}(x, a), \ldots$ will be used for $\partial / \partial x \phi(x, a), \partial / \partial a \phi(. x, a), \ldots$.

Here $a$ may be any particular value oi $x$, and if $b$ is another value of $x$ a second solution of $(1)$ is $\phi(x, b)$, and therefore

$$
\phi(x, a) \phi^{\prime}(x, b)-\phi^{\prime}(x, a) \phi(x, b)
$$

is independent of $x$.

Putting $x=a, b$ in turn, we have

$$
\phi(b, a)=-\phi(a, b) .
$$

Taking $c$ to be a third value of $x$ we have a third solution $\phi(x, c)$ which must be a linear combination of the other two, so that

$$
A \phi(x, a)+B \phi(x, b)+C \phi(x, c)=0,
$$

where $A, B, C$ do not depend on $x$. Their values are found by giving to $x$ the values $a, b$, and thus we have

$$
\phi(x, a) \phi(b, c)+\phi(x, b) \phi(c, a)+\phi(x, c) \phi(a, b)=0 .
$$

From (3) it follows that

so that

$$
\phi^{\prime}(b, a)=-\phi^{\prime}(a, b)
$$

also

$$
\phi^{\prime}(a, a)=-1 \text {; }
$$

so that

$$
\phi^{\prime \prime}(b, a)=-\phi^{\prime \prime}(a, b),
$$$$
\phi^{\prime \prime}(a, a)=0 .
$$

Hence $-\phi^{\prime}(x, a)$ is the solution of (1) which satisfies the initial conditions $\phi=1, d \phi / d x=0$ when $x=a$. We have therefore

$$
\phi(x, a) \phi^{\prime \prime}(x, a)-\phi^{\prime}(x, a) \phi^{\prime}(x, a)=1 \text {. }
$$

A like notation, $\psi(y, a), \ldots$ will be used for the solutions of (2).

As an illustration take the equation

where $k$ is constant. Here

$$
d \ddot{d x^{2}}+\cdots \infty=0
$$

$$
\begin{aligned}
\phi(x, a) & =\frac{1}{k} \sin k(x-a), & \phi^{\prime}(x, a) & =\cos k(x-a), \\
\phi^{\prime}(x, a) & =-\cos k(x-a), & \phi^{\prime \prime}(x, a) & =k \sin k(x-a) .
\end{aligned}
$$

If $\phi x$ denotes any solution of the equation (1), and we suppose $x$ to follow a path beginning at $c$ and ending at $b$, the final values $\phi b, \phi^{\prime} b$ depend on the initial values $\phi a, \phi^{\prime} a$, and are in fact derived from them by a homogeneous linear transformation whose matrix is

$$
\begin{array}{ll}
-\phi^{\prime}(b, a), & \phi(b, a)) \\
-\phi^{\prime \prime}(b, a), & \phi(b, a)
\end{array}
$$


Calling this $W(b, a)$, we have

and the initial condition $W(a, a)=1$.

$$
\frac{d}{d x} W(x, a)=\left\{\begin{array}{cc}
0, & 1 \\
-\lambda S+\mu T-X, & 0
\end{array} \mid W(x, a),\right.
$$

If $W(b, a)$ and $W(c, b)$, the matrices corresponding to the paths $a b, b c$ are known, the matrix for the whole path $a c$ is given by the relation

$$
W(c, a)=W(c, b) W(b, a),
$$

which holds for the solutions of the equation

$$
\frac{d W}{d x}=\mathbb{M} W
$$

where $W, M$ may be matrices of any, the same, order whose constituents are functions of $x$, and $M$ is supposed known, $W$ unknown.

If the equs tion is .

$$
\frac{d W}{d x}=W M
$$

the corresponding relation is $W(c, a)=W(b, a) W(c, b)$, the initial condition being still $W(a, a)=1$.

When $W, M$ are single functions these relations are immediate consequences of the relation

$$
\exp u \exp v=\exp (u+v),
$$

and when they are matrices there is no difficulty in the proof.

\section{Functions $\phi$ with Different Parameters.}

3. In $\$ \S 3-6$ there is not much that is new in substance and the methods and results are therefore not given at great length.

It is known that $\phi(x, a), \psi(y, a)$ and their derivatives are analytical functions of the parameters $\lambda, \mu$, having no singularity except at infinity, that is, are integral functions. This is true even when $S, T, U, V, X, Y$ are not analytical functions, but only integrable; the following method of proof enables us to find their expansions.in powers of $\lambda, \mu$, as well as other results of importance.

Suppose $\phi$ to become $\phi_{1}$ when $\lambda, \mu$ are turned into $\lambda_{1}, \mu_{1}$, then we have $\phi(x, a) \phi_{1}^{\prime \prime}(x, b)-\phi_{1}(x, b) \phi^{\prime \prime}(x, a)=\left\{\left(\lambda-\lambda_{1}\right) S-\left(\mu-\mu_{1}\right) T\right\} \phi(x, a) \phi_{1}(x, b)$, and therefore by integration between the limits $c, u$, $\phi(u, a) \phi_{1}^{\prime}(u, b)-\phi_{1}(u, b) \phi^{\prime}(u, a)-\phi(c, a) \phi_{1}^{\prime}(c, b)+\phi_{1}(c, b) \phi^{\prime}(c, a)$
$=\left(\lambda-\lambda_{1}\right) \int_{c}^{u} S \phi(x, a) \phi_{1}(x, b) d x-\left(u-\mu_{1}\right) \int_{c}^{u} T \phi(x, a) \phi_{1}(x, b) d x$.

The substitution of 0,1 for $a, b, c, u$ in this gives an important series of relations, as in I., pp, 85, 86. Making $\lambda-\lambda_{1}$ small and $\mu=\mu_{1}$, we have

$$
\begin{array}{r}
\phi(u, a) \frac{\partial}{\partial \lambda} \phi^{\prime}(u, b)-\phi^{\prime}(u, a) \frac{\partial}{\partial \lambda} \phi(u, b)-\phi(c, a) \frac{\partial}{\partial \lambda} \phi^{\prime}(c, b)+\phi^{\prime}(c, a) \frac{\partial}{\partial \lambda} \phi(c, b) \\
=-\int_{c}^{u} S \phi(x, a) \phi(x, b) d x ;
\end{array}
$$


and on putting $u=a, c=b$,

$$
\frac{\partial}{\partial \lambda} \phi(a, b)=-\int_{b}^{a} S \phi(a, x) \phi(x, b) d x
$$

from which the more general result could be derived after differentiation as to $a, b$. Similarly,

$$
\frac{\partial}{\partial \mu} \phi(a, b)=\int_{b}^{a} T \phi(a, x) \phi(x, b) d x .
$$

The higher derivatives may also be found ; the general formula is

$$
\begin{aligned}
& \frac{\partial^{n}}{\partial \lambda^{n-r} \partial \mu^{r}} \Phi\left(x_{0}, x_{n+1}\right)=\text { the coefficient of } \kappa^{n-r} \text { in } \\
& \qquad(n-r) ! r ! \iiint \ldots \prod_{p=1}^{n}\left(T_{p}-\kappa S_{p}\right) \prod_{p=0}^{n} \phi\left(x_{p}, x_{p+1}\right) \prod_{p=1}^{n} d x_{p},
\end{aligned}
$$

the integration being over all such values that $x_{0}, x_{1}, x_{2}, \ldots, x_{n}, x_{n+1}$ lie in reverse order on the path chosen from $x_{n+1}$ to $x_{0}$ : that is, we are to integrate first as to $x_{1}$ from $x_{2}$ to $x_{0}$, then as to $x_{2}$ from $x_{3}$ to $x_{0}$, and so on.

$$
\text { Reality of Solutions of } \theta=0, Z=0 \text {. }
$$

4. Now let $E, G, E_{1}, \ldots, E_{4}, G_{1}, \ldots, G_{4}$ be real constants, and write $\theta, Z$ for

$$
\begin{aligned}
& E_{1} \phi(1,0)+E_{2} \phi^{\prime}(1,0)+E_{3} \phi^{\prime}(1,0)+E_{4} \phi^{\prime \prime}(1,0)-2 E, \\
& G_{1} \psi(1,0)+G_{2} \psi^{\prime}(1,0)+G_{3} \psi^{\prime}(1,0)+G_{4} \psi^{\prime \prime}(1,0)-2 G,
\end{aligned}
$$

respectively, so that $\theta, Z$ are integral functions of $\lambda, \mu$. It is to be proved that the values of $\lambda, \mu$ for which $\theta=0, Z=0$ are real and generally distinct, if the coefficients $E_{1}, G_{1}, \ldots$ satisfy certain irequalities. With the notation of $\$ 3$, we have (as in I., pp. 87, 103)

$\Theta\left\{E_{3} \phi_{1}(1,0)+E_{4} \phi_{1}^{\prime}(1,0)\right\}-\Theta_{1}\left\{E_{3} \phi(1,0)+E_{4} \phi^{\prime}(1,0)\right\}$

$$
\begin{aligned}
=\int_{0}^{1} & {\left[\left\{E_{3} \phi(x, 1)+E_{4} \phi^{\prime}(x, 1)+E \phi(x, 0)\right\}\right.} \\
& \times\left\{E_{3} \phi_{1}(x, 1)+E_{4} \phi_{1}^{\prime}(x, 1)+E \phi_{1}(x, 0)\right\} \\
& \left.+\left(E_{1} E_{4}-E_{2} E_{3}-E^{2}\right) \phi^{\prime}(x, 0) \phi_{1}(x, 0)\right]\left\{\left(\lambda-\lambda_{1}\right) S-\left(\mu-\mu_{1}\right) T_{;}^{\prime} d x .\right.
\end{aligned}
$$

Suppose that $\theta=0$ for values of $\lambda, \mu$ that are not real, and let $\lambda_{1}, \mu_{1}$ be conjugate imaginaries to $\lambda, \mu$; then $\Theta_{1}$ is conjugate to $\Theta$ and must vanish, so that the left-hand side of (5) is zero. Also $\phi(x, 0)$ sind $\phi_{1}(x, 0), \ldots$, aro pairs of conjugates, and thus if $E_{1} E_{4^{*}}-E_{2} E_{3}-Z^{2}$ is positive the expression in square brackets on the right in (5) is always positive. Since the whole integral varizhes the purely imaginary factor 
$\left(\lambda-\lambda_{1}\right) S-\left(\mu-\mu_{1}\right) T$ cannot be of constant sign, but $\left(\lambda-\lambda_{1}\right) /\left(\mu-\mu_{i}\right)$ must be a mean among the values of $T / S$.

If $Z=0$ for the same values of $\lambda$, $\mu$, and if $G_{1} G_{4}-G_{2} G_{3}-G^{2}$ is positive, it follows in like manner that $\left(\lambda-\lambda_{1}\right) /\left(\mu-\mu_{1}\right)$ must be a mean among the values of $V / U$. Hence $\theta, Z$ cannot both vanish except for real values of $\lambda, \mu$, since the greatest value of $T / S$ is less than the least of $V / U$.

The same conclusion follows if $E_{1} E_{4}-E_{2} E_{9}-E^{2}=0$; see I., p. 87 . We shall assume that $E_{1} E_{4}-E_{2} E_{3}-E^{2}$ and $G_{1} G_{4}-G_{2} G_{3}-G^{2}$ are positive or zero so that the equations $\theta=0, Z=0$ cannot both be satisfied by imaginary values of $\lambda, \mu$.

\section{Distinctness of Solutions of $\theta=0, Z=0$.}

5. The values of $\lambda, \mu$ given by the equations $\theta=0, Z=0$ are generally distinct as well as real. If they were not, the Jacobian $\partial(\theta, Z) / \partial(\lambda, \mu)$ would vanish as well as $\theta, Z$. But we have

$$
\begin{aligned}
\frac{\partial \Theta}{\partial \lambda}=-\int_{0}^{1}\left\{E_{1} \phi(1, x) \phi(x, 0)+E_{2} \phi^{\prime}(1, x) \phi(x, 0)\right. & +E_{8} \phi(1, x) \phi^{\prime}(x, 0) \\
& \left.+E_{4} \phi^{\prime}(1, x) \phi^{\prime}(x, 0)\right\} S d x,
\end{aligned}
$$

with like expressions for the other derivatives* and hence

$$
\begin{array}{r}
\frac{\partial(\Theta, Z)}{\partial(\lambda, \mu)}=\int_{0}^{1} \int_{0}^{1}\left\{E_{1} \phi(1, x) \phi(x, 0)+E_{2} \phi^{\prime}(1, x) \phi(x, 0)\right. \\
+E_{3} \phi(1, x) \phi^{\prime}(x, 0) \\
\left.+E_{4} \phi^{\prime}(1, x) \phi^{\prime}(x, 0)\right\} \\
\times\left\{G_{1} \psi(1, y) \psi(y, 0)+G_{2} \psi^{\prime}(1, y) \psi(y, 0)+G_{3} \psi(1, y) \psi^{\prime}(y, 0)\right. \\
\left.+G_{4} \psi^{\prime}(1, y) \psi^{\prime}(y, 0)\right\}(S V-U T) d y d x .
\end{array}
$$

\footnotetext{
- A more general formula is $\Theta \delta \theta-\theta \delta \Theta=-\int_{0}^{1}\left\{A[\phi(x, 0)]^{2}+2 B \phi(x, 0) \phi^{\prime}(x, 0)+C\left[\phi^{\prime}(x, 0)\right]^{2}\right\}(S \delta \lambda-T \delta \mu) d x$, where $\theta=\epsilon_{1} \phi(1,0)+e_{2} \phi^{\prime}(1,0)+e_{3} \phi^{\prime}(1,0)+e_{4} \phi^{\prime \prime}(1,0)-2 e$, $A=(12)-(13) \phi^{\prime 2}-(14) \phi^{\prime} \phi^{\prime \prime}-(23) \phi^{\prime} \phi^{\prime \prime}-(24) \phi^{\prime \prime 8}+(10) 2 \phi^{\prime}+(20) 2 \phi^{\prime \prime}$, $B=(13) \phi \phi^{\prime}+(14) \phi \phi^{\prime \prime}+(23) \phi^{\prime} \phi^{\prime}+(24) \phi^{\prime} \phi^{\prime \prime}-(10) \phi-(20) \phi^{\prime}+(30) \phi^{\prime}+(40) \phi^{\prime \prime}$, $C=(34)-(13) \phi^{2}-(14) \phi \phi^{\prime}-(23) \phi \phi^{\prime}-(24) \phi^{\prime 2}-(30) 2 \phi-(40) 2 \phi^{\prime}$, $(12)=E_{1} e_{2}-e_{1} E_{2}, \quad(30)=E_{3} e-e_{3} E, \quad \ldots$, $\phi, \phi^{\prime}, \phi^{\prime}, \phi^{\prime \prime}=\phi(1,0), \phi^{\prime}(1,0), \phi^{\prime}(1,0), \phi^{\prime \prime}(1,0)$ respectively.
}

The quadratic in $\phi(x, 0)$ and $\phi^{\prime}(x, 0)$ has for its discriminant

$A C-B^{2}=\left(E_{1} E_{4}-E_{2} E_{3}-E^{2}\right) \theta^{2}-\left(E_{1} e_{4}+E_{4} e_{1}-E_{2} e_{3}-E_{9} e_{2}-2 \dot{E} e\right) \theta \theta+\left(e_{1} e_{4}-e_{2} e_{3}-\iota^{\prime}\right) \Theta^{2}$, and is therefore of constant sign if

$$
\left(E_{1}+\kappa e_{1}\right)\left(E_{4}+\kappa e_{4}\right)-\left(E_{2}+\kappa e_{2}\right)\left(E_{3}+\kappa e_{3}\right)-(E+\kappa e)^{2}
$$

is never negative for any real value of $\boldsymbol{~}$.

SER. 2. VOL. 5. No. 970 . 
In this expression the subject of integration has a constant sign when $\theta, Z$ vanish, for while $S V-U T$ is always positive, the first factor is

$$
\begin{aligned}
& \left.\vdots E_{1} \phi^{\prime}(1,0)+E_{2} \phi^{\prime \prime}(1,0)\right\}[\phi(x, 0)]^{2} \\
& \left.\quad+\vdots-E_{1} \phi(1,0)-E_{2} \phi^{\prime}(1,0)+E_{3} \phi^{\prime}(1,0)+E_{4} \phi^{\prime \prime}(1,0)\right\} \phi(x, 0) \phi^{\prime}(x, 0) \\
& \quad+\left\{-E_{3} \phi(1,0)-E_{4} \phi^{\prime}(1,0)\right\}\left[\phi^{\prime}(x, 0)\right]^{2}, \text { say } H,
\end{aligned}
$$

a quadratic in $\phi(x, 0), \phi^{\prime}(x, 0)$ whose discriminant is

$$
4\left(E_{1} E_{4}-E_{2} E_{3}\right)-(\Theta+2 E)^{2},
$$

so that $H$ is of constant sign when $\theta=0$; the second factor can be similarly expressed.

Hence the Jacobian can only vanish when one factor or the other vanishes for all values of $x, y$, so that we have either

$$
E_{1}=E \phi^{\prime \prime}(1,0), \quad E_{2}=-E \phi^{\prime}(1,0), \quad E_{3}=-E \phi^{\prime}(1,0), \quad E_{4}=E \phi(1,0),
$$

$$
E_{1} E_{4}-E_{2} E_{3}-E^{2}=0, \quad E \neq 0, \quad \partial \theta / \partial \lambda=0=\partial \theta / \partial \mu,
$$

or else $\quad G_{1}=G \psi^{\prime \prime}(1,0), \ldots, \quad \partial Z / \partial \lambda=0=\partial Z / \partial \mu$.

(Compare I., p. 88.)

Thus, if $\lambda, \mu$ are taken as Cartesian coordinates, the two curves $\theta=0$, $Z=0$ have their intersections all distinct unless one passes through a node on the other. Neither curve can have a triple point, for

$$
\begin{aligned}
\frac{\hat{\partial}^{2} \Theta}{\partial \lambda^{2}}= & 2 \int_{0}^{1} \int_{x}^{1} i E_{1} \phi\left(1, x_{1}\right) \phi\left(x_{1}, x\right) \phi(x, 0)+E_{2} \phi^{\prime}\left(1, x_{1}\right) \phi\left(x_{1}, x\right) \phi(x, 0) \\
& \left.+E_{3} \phi\left(1, x_{1}\right) \phi\left(x_{1}, x\right) \phi^{\prime}(x, 0)+E_{4} \phi^{\prime}\left(1, x_{1}\right) \phi\left(x_{1}, x\right) \phi^{\prime}(x, 0)\right\} S S_{1} d x_{1} d x,
\end{aligned}
$$

which reduces to $\quad-2 E \int_{0}^{1} \int_{x}^{1}\left[\phi\left(x_{1}, x\right)\right]^{2} S S_{1} d x_{1} d x$

or

$$
-E \int_{0}^{1} \int_{0}^{1}\left[\phi\left(x_{1}, x\right)\right]^{2} S S_{1} d x_{1} d x
$$

when

$$
E_{1}=E \phi^{\prime \prime}(1,0), \quad E_{2}=-E \phi^{\prime}(1,0), \quad \ldots,
$$

that is, when the curve $\theta=0$ has a double point.

$$
\text { Similarly } \begin{aligned}
\frac{\partial^{2} \Theta}{\partial \mu^{2}} & =-E \int_{0}^{1} \int_{0}^{1}\left[\phi\left(x_{1}, x\right)\right]^{2} T T_{1} d x_{1} d x, \\
\frac{\partial^{2} \Theta}{\partial \lambda \partial \mu} & =E \int_{0}^{1} \int_{0}^{1}\left[\phi\left(x_{1}, x\right)\right]^{2} S T_{1} d x_{1} d x .
\end{aligned}
$$


These second derivatives cannot vanish, so that there can be no triple points. In the case of a double point

$$
\begin{aligned}
& a^{2} \frac{\partial^{2} \Theta}{\partial \lambda^{2}}+2 \alpha \beta \frac{\partial^{2} \Theta}{\partial \lambda \partial \mu}+\beta^{2} \frac{\partial^{2} \Theta}{\partial \mu^{2}} \\
& \quad=-E \int_{0}^{1} \int_{0}^{1}\left[\phi\left(x_{1}, x\right)\right]^{2}(\alpha S-\beta T)\left(\alpha S_{1}-\beta T_{1}\right) d x_{1} d x,
\end{aligned}
$$

which cannot vanish if $\alpha S-\beta T$ is of constant sign.

Hence on each branch $d \mu / d \lambda$, if real, must be a mean among the values of $S / T$. Also $d \mu / d \lambda$ must be real, for, if not, the equation $\theta=0$ could be satisfied by giving $\lambda$ a consecutive real value and $\mu$ an imaginary one, which we know to be impossible, since the ratio of the imaginary parts of $\lambda, \mu$ is a mean among the values of $T / S$, and $T, S$ are always positive.* For the same reason if the values of $d \mu / d \lambda$ are equal, the point must be a tacnode and not a cusp.

A tacnode is not impossible, nor is any order of contact between real branches of the curve $\theta=0$ impossible, as may best be seen by the example of the differential equation

$$
\phi^{\prime \prime}+(\lambda-\mu) \phi=0,
$$

when

$$
E_{1}=0=E_{4}, \quad E_{2}=-E_{3}=E=1 .
$$

We then have $\quad \phi(1,0)=(\lambda-\mu)^{-\frac{1}{2}} \sin (\lambda-\mu)^{\frac{1}{2}}$,

$$
\begin{gathered}
\phi^{\prime}(1,0)=-\phi^{\prime}(1,0)=\cos (\lambda-\mu)^{\frac{3}{2}}, \\
\Theta=\phi^{\prime}(1,0)-\phi^{\prime}(1,0)-2=2 \cos (\lambda-\mu)^{\frac{3}{2}}-2,
\end{gathered}
$$

so that the curve $\theta=0$ consists of the straight line $\lambda=\mu$ counted once,

- The following is another proof. Let

$$
\alpha=\int_{0}^{1} T[\phi(x, 0)]^{2} d x, \quad \beta=\int_{0}^{1} S[\phi(x, 0)]^{2} d x,
$$

then $\alpha^{2} \frac{\partial^{2} \Theta}{\partial \lambda^{2}}+2 \alpha \beta \frac{\partial^{2} \Theta}{\partial \lambda \partial \mu}+\beta^{2} \frac{\partial^{2} \Theta}{\partial \mu^{2}}$

$$
\begin{gathered}
=-E \int_{0}^{1} \int_{0}^{1} \int_{0}^{1} \int_{0}^{1}\left[\left\{\phi\left(x, x_{1}\right) \phi\left(x_{2}, 0\right) \phi\left(x_{3}, 0\right)\right\}^{2}-\left\{\phi\left(x, x_{2}\right) \phi\left(x_{1}, 0\right) \phi\left(x_{3}, 0\right)\right\}^{2}\right. \\
\left.-\left\{\phi\left(x_{1}, x_{3}\right) \phi\left(x_{2}, 0\right) \phi(x, 0)\right\}^{2}+\left\{\phi\left(x_{2}, x_{3}\right) \phi(x, 0) \phi\left(x_{1}, 0\right)\right\}^{2}\right] \\
\times S S_{1} T_{2} T_{3} d x d x_{1} d x_{2} d x_{3}
\end{gathered}
$$

a quantity which is of opposite sign to the coefficient of $a^{2}$ in the quadratic form, or else is zero. Hence the factors of the form are real. 
and the lines $\lambda=\mu+4 n^{2} \pi^{2}$, each counted twice, where $n=1,2,3,4, \ldots$; any point on a repeated branch must be reckoned as a point of contact of two branches.

At any ordinary point of the curve $\theta=0$, the value of $d \mu / d \lambda$ is also a mean among the values of $S / T$, for $\alpha \frac{\partial \theta}{\partial \lambda}+\beta \frac{\partial \theta}{\partial \mu}$ is the integral of the product when $-\alpha S+\beta T$ is multiplied by the quadratic $H$ in $\phi(x, 0)$, $\phi^{\prime}(x, 0)$, which has been proved to be of constant sign. Similarly on the curve $Z=0, d \mu / d \lambda$ is always a mean among the values of $U / V$, and thus the curves cut at a finite angle whether at an ordinary or a double point.

\section{A certain Double Integral.}

6. The main question we have to discuss relates to the double integral

$$
\iint \frac{\Phi(x, t) \Psi(y, u)}{\Theta Z} d \mu d \lambda
$$

where $\Phi(x, t)=E_{1} \phi(1, t) \phi(x, 0)+E_{2} \phi^{\prime}(1, t) \phi(x, 0)$

$$
+E_{3} \phi(1, t) \phi^{\prime}(x, 0)+E_{4} \phi^{\prime}(1, t) \phi^{\prime}(x, 0)+E \phi(t, x),
$$$$
\Psi(y, u)=G_{1} \psi(1, u) \psi(y, 0)+G_{2} \psi^{\prime}(1, u) \psi(y, 0)
$$$$
+G_{3} \psi(1, u) \psi^{\prime}(y, 0)+G_{4} \psi^{\prime}(1, u) \psi^{\prime}(y, 0)+G \psi(u, y) .
$$

Since $\quad \phi(1, t) \phi(x, 0)-\phi(1, x) \phi(t, 0)=\phi(1,0) \phi(x, t)$,

it will be seen that

$$
\Phi(x, t)-\Phi(t, x)=\theta \phi(x, t),
$$

and that when $\Theta$ vanishes $\Phi$ is a symmetric function of $x, t$; similarly

$$
\Psi(y, u)-\Psi(u, y)=Z \psi(y, u)
$$

so that $\Psi$ is a symmetric function when $Z=0$.*

- The following are properties of $\Phi, \Theta$ given nere for reference.

$$
\begin{aligned}
& \begin{array}{l}
\partial \Theta \\
\partial \lambda
\end{array}=-\int_{0}^{1} S \Phi(x, x) d x, \quad \frac{\partial \Theta}{\partial \mu}=\int_{0}^{1} T \Phi(x, x) d x, \\
& \grave{j}_{j \lambda}^{\partial} \Phi(x, t)=-\int_{t}^{1} S_{1} \Phi\left(x, x_{1}\right) \phi\left(x_{1}, t\right) d x_{1}-\int_{0}^{x} S_{1}^{\prime} \Phi\left(x_{1}, t\right) \phi\left(x, x_{1}\right) d x_{1}+E \int_{0}^{1} S_{1} \phi\left(x_{1}, x\right) \phi\left(x_{1}, t\right) d x_{1} \text {, }
\end{aligned}
$$

and so for $i \Phi / \partial \mu$. Where there is a node on the curve $\Theta=0, \Phi=0$ for all values of $x, t$, and

$$
\delta \Phi(x, t)=L \int_{0}^{1}\left(S_{1} \delta \lambda-T_{1} \delta \mu\right) \phi\left(x_{1}, x\right) \phi\left(x_{1}, t\right) d x_{1},
$$

from which could be found the values already given $(\$ j)$ for the second derivatives of $\Theta$. 
Since $\Phi, \Psi, \theta, Z$ are integral functions of $\lambda, \mu$, the value of the double integral, when taken over a closed region of two dimensions, will depend on the situation of that region in relation to the values of $\lambda, \mu$ for which $\theta Z$ vanishes, and will be unaltered by continuous deformation of that region so long as it contains no point where $\Theta Z$ vanishes. (Picard and Simart, Fonctions algébriques de deux variables indépendantes, Vol. I., ch. iii.)

\section{Known Results relating to Double Contour Integrals.}

6a. Suppose that in the second or outer integration, with respect to $\lambda$, the path* is a closed contour $L$, and that to each point of $L$ there corresponds a certain closed path of integration $M$, in the $\mu$-plane: as $\lambda$ moves round $L, M$ may vary continuously, but its final position must coincide with its initial one, and in no position may it pass through any of the points where $\theta$ vanishes, say $\mu^{\prime}$, or the points where $Z$ vanishes, say $\mu^{\prime \prime}$.

For certain values of $\lambda$ there will be coincidences among the points $\mu^{\prime}, \mu^{\prime \prime}$, and if $L$ encloses none of these values of $\lambda$, the value of the double integral will be zero. If such a point $\lambda_{r}$ is enclosed, it may be (1) a branch point for $\mu^{\prime}$ (or $\mu^{\prime \prime}$ ), or (2) correspend to a double point on the curve $\theta=0$ (or $Z=0$ ), or (3) be such that $\theta, Z$ vanish for a common value of $\mu$, say $\mu_{r}$, when $\lambda=\lambda_{r}$. These three alternatives do not exclude each other.

In case (1) the effect will depend on the position of the value $\mu^{\prime}$ (or $\mu^{\prime \prime}$ ), affected by the branch point, in regard to the contour $M$. In the initial position $M$ will, in general, enclose some of the points $\mu^{\prime}, \mu^{\prime \prime}$, and exclude others. As $\lambda$ travels round $L$ the points $\mu^{\prime}, \mu^{\prime \prime}$ describe paths which, singly or together, form one or more closed circuits. The circuits described by the enclosed points will be separate from those described by the excluded points, since the curve $M$ separates the two sets of points in all positions. (This does not hinder an excluded point from taking a position which has formerly been occupied by an enclosed point, since the value of $\lambda$ has changed.)

Now, if $L$ encloses a branch point which affects only enclosed points, or only excluded points, the resulting contribution to the double integral is zero. If, on the other hand, $L$ contains branch points which permute enclosed with excluded points, one or more of the circuits described by

* Each of the complex variables $\lambda, \mu$ is of course represented by a point in a separate plane, as in Argaud's diagram. 
the enclosed points may be irreducible, and thence will arise a contribution equal to a period of a certain single integral of the Abelian type.

As an illustration of the effect of branch points take

$$
\iint\left(\lambda^{4}+\mu^{2}-1\right)^{-1} d \mu d \lambda,
$$

where the problem is somewhat simplified, but not essentially changed, by the absence of any second factor in the denominator of the subject of integration. Here there are two values of $\mu^{\prime}$, namely $\pm\left(1-\lambda^{4}\right)^{3}$, and they are permuted by a circuit round any one of the branch points in the $\lambda$-plane, $\pm 1, \pm \imath$.

Hence, if $L$ consists of a simple contour enclosing one of these branch points, $M$ must enclose both or neither of the values $\mu^{\prime}$; otherwise its final position could not be the eame as its initial one, and the region defined would not be closed. If, however, $L$ encircles two branch points, or the sume one twice, $M I$ may be taken a simple contour enclosing one of the $\mu^{\prime}$ points, say $\left(1-\lambda^{4}\right)^{3}$.

The integration with respect to $\mu$ now gives the result

$$
2 \iota \pi \int_{L} \frac{1}{2 \mu^{\prime}} d \lambda \text { or } \iota \pi \int_{L}\left(1-\lambda^{4}\right)^{-d} d \lambda,
$$

and this, when $L$ encircles two branch points, is one of the periods of an Abelian integral associated with the equation

$$
\lambda^{4}+\mu^{2}-1=0 \text {. }
$$

In case (3) when $\theta, Z$ vanish together, the result may be stated in the same way, the Abelian integral being of the third kind, and the period that associated with a contour encircling its parametric points. The value of the contribution is, however, capable of being directly expressed in a simple form.

Suppose $\lambda$ to leave the path $L$ and travel inwards to $\lambda_{r}$, the point under discussion, without passing through any position in which there is a coincidence among the points $\mu^{\prime}, \mu^{\prime \prime}$. As $\lambda$ travels we can deform $M$ continuously, so that $M$ does not pass through a $\mu^{\prime}$ or a $\mu^{\prime \prime}$ until $\lambda$ actually reaches $\lambda_{r}$. Then, if at $\lambda_{r}$ an enclosed $\mu^{\prime}$ comes into coincidence with an excluded $\mu^{\prime \prime}$ or conversely, it ceases to be possible to make $M$ pass between them, and there will be a contribution to the value of the double integral of

where

$$
\pm(2 \iota \pi)^{2} \Omega\left(\lambda_{r}, \mu_{r}\right)
$$

$$
\Omega=\Phi(x, t) \Psi(y, u) / \frac{\partial(\Theta, Z)}{\partial(\lambda, \mu)}
$$

(in general, the numerator of the subject of integration divided by the Jacobian of the factors of the denominator). The lower sign is to be taken when $\mu^{\prime \prime}$ is excluded, the upper when it is enclosed.

When the coincidence is between an enclosed $\mu^{\prime}$ and an enclosed $\mu^{\prime \prime}$, or between an excluded $\mu^{\prime}$ and an excluded $\mu^{\prime \prime}$, there is no contribution, and the coincidence does not hinder us from drawing $M$ in the way prescribed. 
Case (2) is essentially the same as case (3), for, when the curve $\theta=0$ has a node at $\left(\lambda_{r}, \mu_{r}\right), \theta$ may be expressed as the product of two functions, each without singularity in the domain of $\left(\lambda_{r}, \mu_{r}\right)$, and each vanishing at that point to the first order only.

\section{General Idea of the Proposed Application of these Results.}

6b. The values of $\lambda$ for which a $\mu^{\prime}$ coincides with a $\mu^{\prime \prime}$ form an infinite series, and it will be our object so to choose the region of integration that the value of the double integral will be expressed by the corresponding series $\Sigma \Omega$, multiplied by $(2 \iota \pi)^{2}$. We must avoid the possibility of contributions from branch points as in case (1), and take in all possible contributions under case (3).

The general idea will be to have the $\mu^{\prime \prime}$ points, where $Z$ vanishes, enclosed by $M$, and the $\mu^{\prime}$ points, where $\theta$ vanishes, excluded. If there is a node on $Z=0$, so that two $\mu^{\prime \prime}$ points coincide, this will make no difference in the form of the result, since the coincidence will be between two enclosed points; and similarly if the node is on $\theta=0$. For the present we shall suppose the intersections of the two curves to be simple, so as to avoid complications that fall beyond the scope of the account that has been given.

It will be noticed that the terms of the series $\Sigma \Omega$ are symmetric functions of $x, t$ as well as of $y, u$.

In order to decide on a suitable region of integration, we must study the distribution of the values of $\mu$ for which $\theta, Z$ vanish severally when $\lambda$ has a fixed value, and more particularly when that value is great and not real. With a view to this we find approximate values for $\phi, \psi$ and their derivatives.

As an illustration of the use to be made of the double integral here given, it may be pointed out that the Fourier expansion in a series of sines depends on the series

$$
\sum_{n=1}^{\infty} \sin n \pi x \sin n \pi t
$$

which is the series of periods of the integral

$$
-\frac{1}{4} \iota \pi \int \frac{\sin (x \sqrt{ } \lambda) \sin \{(1-t) \sqrt{ } \lambda\}}{\sqrt{\lambda} \sin \sqrt{ } \lambda} d \lambda .
$$

Here, again, the subject of integration is unsymmetrical, but the residues are symmetrical, as functions of $x$ and $t$. The subject of integration, moreover, is very small when $\lambda$ is great and complex or negative, provided $x<t$. The object of my former paper (I.) was to generalize this expression and apply it to the series of Liouville and Sturm for functions of one variable. It is now to be further extended to functions of two variables. 
Approximations by means of Hyperbolic Functions when $\lambda, \mu$ are Great.

7. If we write $P(x)$ for $-\lambda S+\mu T-X$, and suppose $\lambda, \mu$ to be great, the following formulæ are, in general, approximately true.*

$$
\left.\begin{array}{c}
\phi(b, a)=\{P(a) P(b)\}^{-\frac{1}{2}} \sinh \int_{a}^{b} P^{\frac{1}{b}} d x \\
\phi^{\prime}(b, a)=\{P(b) / P(a)\}^{\frac{1}{2}} \cosh \int_{a}^{b} P^{\frac{b}{b}} d x \\
\phi^{\prime}(b, a)=-\{P(a) / P(b)\}^{\frac{1}{2}} \cosh \int_{a}^{b} P^{\frac{1}{b}} d x \\
\phi^{\prime \prime}(b, a)=-\{P(a) P(b)\}^{\frac{1}{t}} \sinh \int_{a}^{b} P^{\frac{1}{b}} d x
\end{array}\right\} .
$$

In proving these formulæ and discussing the restrictions under which they hold, we shall use the following lemma, which is closely related to the formulm of $\S 3$.

Let $f(x, a)$ denote the function which satisfies the equation

$$
\frac{d^{2} f}{d x^{2}}=Q f
$$

and the further conditions that when $x=a, f=0$, and $d f / d x=1$. Let $Q$ be changed to $Q+\delta Q$, and suppose $\delta f$ to denote the consequent increase in $f$. Then

$$
\begin{aligned}
\delta f(b, a) & =\int_{a}^{b} f(b, x)\{f(x, a)+\delta f(x, a)\} \delta Q d x \\
& =\int_{a}^{b}\{f(b, x)+\delta f(b, x)\} f(x, a) \delta Q d x .
\end{aligned}
$$

For

$$
f^{\prime \prime}(x, b)=Q f(x, b)
$$

and

$$
f^{\prime \prime}(x, a)+\delta f^{\prime \prime}(x, a)=(Q+\delta Q)\{f(x, a)+\delta f(x, a)\} .
$$

* Accurataly

$$
\phi(b, a)=\left(\sigma^{\prime} a \sigma^{\prime} b\right)^{-1} \sinh (\sigma b-\sigma a),
$$

where $\sigma x$ is a function defined by the equation

$$
\left(\sigma^{\prime} x\right)^{2}-\frac{1}{2}\{\sigma x, x\}=P(x) .
$$

If we put $y$ for $\left(\sigma^{\prime} x\right)^{-i}$, this equation becomes

$$
y^{\prime \prime}+1 / y^{3}=y P(x),
$$

which, with the slightly more general $y^{\prime \prime}+e / y^{3}=y P(x)$,

may be added to the list of forms associated with the linear equation of the second order, and which, like the linear equation, is such that, if one solution is known, all the rest can be deduced.

If $\phi_{1}, \phi_{2}$ are two solutions of (1) and are such that $\phi_{1}^{\prime} \phi_{2}-\phi_{2}^{\prime} \phi_{1}=1$, then the most general value of $y$ is $\left(\Delta \phi_{1}^{2}+2 B \phi_{1} \phi_{2}+C \phi_{2}^{2}\right)^{t}$, where $A, B, C$ are constants such that $B^{2}-A C=e$ : a value of $\sigma x$ is $\tanh ^{-1} \phi_{1} / \phi_{2}$. 
1906.] HARMONIC EXPANGIONS OF FUNCTIONS OF TWO VARIABLFS.

\section{Hence}

$f^{\prime \prime}(x, b)\{f(x, a)+\delta f(x, a)\}-f(x, b)\left\{f^{\prime \prime}(x, a)+\delta f^{\prime \prime}(x, a)\right\}$

Hence

$$
=-\delta Q f(x, b)\{f(x, a)+\delta f(x, a)\} \text {. }
$$

$$
\begin{aligned}
& \int_{a}^{b} f(b, x)\{f(x, a)+\delta f(x, a)\} \delta Q, d x \\
& =\left.\right|_{x=a} ^{x=b}\left[f^{\prime}(x, b)\{f(x, a)+\delta f(x, a)\}-f(x, b)\left\{f^{\prime}(x, a)+\delta f^{\prime}(x, a)\right\}\right] \\
& =[f(b, a)+\delta f(b, a)]-[-f(a, b)]=\delta f(b, a), \\
& f(a, a)=f(b, b)=0 \\
& f^{\prime}(a, a)=f^{\prime}(b, b)=1=f^{\prime}(a, a)+\delta f^{\prime}(a, a) ; \\
& \delta f^{\prime}(a, a)=0 .
\end{aligned}
$$

Interchanging $a, b$ in the result, we have the second form (8) for $\delta f(b, a)$.

Now, in order that $f(b, a)$ may, for all values of $a, b$, have the value

$$
\{P(a) P(b)\}^{-\frac{1}{d}} \sinh \int_{a}^{b} P^{\frac{1}{d}} d x,
$$

proposed above as an approximation to $\phi(b, a)$, we must put

$$
Q=P+\frac{5}{16}\left(P^{\prime} / P\right)^{2}-\frac{1}{4} P^{\prime \prime} / P,
$$

since this is then the value of $f^{\prime \prime}(x, a) / f(x, a)$, as may readily be verified. Then, if

$$
\delta Q=-\frac{5}{16}\left(P^{\prime} / P\right)^{2}+\frac{1}{4} P^{\prime \prime} / P,
$$

we have

$$
Q+\delta Q=P \quad \text { and } \quad f+\delta f=\phi .
$$

The error committed when $f(b, a)$ is taken as the value of $\phi(b, a)$ is $\delta f(b, a)$, and an estimate of its magnitude can be formed by the use of the Lemma (7) as follows.

If $\alpha, \beta$ are real quantities,

$$
\sinh (\alpha+\imath \beta) \sinh (\alpha-\imath \beta)=\sinh ^{2} \alpha+\sin ^{2} \beta=\cosh ^{2} \alpha-\cos ^{2} \beta .
$$

Hence $\quad|\sinh (\alpha+\imath \beta)| \geqslant|\sinh \alpha|$ and $\leqslant \cosh \alpha$.

Thus, if the element $P^{\frac{1}{2}} d x$ is separated into a real part $d \rho$ and a purely imaginary part $\iota d \sigma$,

$$
\left|\sinh \int_{a}^{b} P^{\frac{1}{2}} d x\right| \leqslant \cosh \int_{a}^{b} d \rho,
$$

and this again does not exceed cosh $\int_{a}^{b}|d \rho|$, where the integral contains the same elements as $\int_{a}^{b} d \rho$ but all taken positively, and is less than 
$\cosh \int_{a}^{b}|d \rho|$ unless $d \rho$ is of constant sign, since the hyperbolic cosine increases continually with its argument.

Suppose $\eta$ to be the greatest absolute value of

$$
\delta f\left(x_{1}, x_{0}\right) /\left\{P\left(x_{1}\right) P\left(x_{0}\right)\right\}^{-1} \cosh \int_{x_{0}}^{x_{1}}|d \rho|
$$

for all positions of $x_{1}, x_{0}$ on the path from $a$ to $b$.

We have

$\left|f\left(x_{1}, x_{0}\right)\right|=\mid\left\{\left.\left.P\left(x_{1}\right) P\left(x_{0}\right)\right|^{-\frac{1}{2}} \sinh \int_{x_{0}}^{x_{1}} P^{\mathrm{k}} d x|\leqslant| P\left(x_{1}\right) P\left(x_{0}\right)\right|^{-\frac{k}{z}} \cosh \int_{x_{0}}^{x_{1}}|d \rho|\right.$, and therefore

$$
\left|\phi\left(x_{1}, x_{0}\right)\right|=\left|f\left(x_{1}, x_{0}\right)+\delta f\left(x_{1}, x_{0}\right)\right| \leqslant(1+\eta)\left|P\left(x_{1}\right) P\left(x_{0}\right)\right|^{-\frac{1}{d}} \cosh \int_{x_{0}}^{x_{1}}|d \rho| .
$$

But, by (7),

$$
\begin{aligned}
& \begin{array}{r}
|\delta f(b, a)|=\left|\int_{a}^{b} f(b, x) \phi(x, a) \delta Q d x\right| \\
\leqslant \int_{a}^{b}|f(b, x)||\phi(x, a)||\delta Q||d x| \\
\leqslant \int_{a}^{b}|P(b) P(x) P(x) P(a)|^{-\frac{1}{b}} \cosh \int_{x}^{b}|d \rho|(1+\eta) \cosh \int_{a}^{x}|d \rho||\delta Q||d x| . \\
\text { Now } \quad \int_{x}^{b}|d \rho|+\int_{a}^{x}|d \rho|=\int_{a}^{b}|d \rho|,
\end{array}
\end{aligned}
$$

and therefore $\cosh \int_{x}^{b}|d \rho| \cosh \int_{a}^{x}|d \rho|<\cosh \int_{a}^{b}|d \rho|$.

Let $M$ be the greatest value of $\left|P^{-h} \delta Q\right|$ and $h$ the length of the path of integration, that is, $\int_{a}^{b}|d x|$. Then we have

$$
|\delta f(b, a)|<(1+\eta)|P(b) P(a)|^{-\frac{1}{b}} \cosh \int_{a}^{b}|d \rho| M h,
$$

and the same is clearly true if intermediate values $x_{1}, x_{0}$ are put in the places of $b, a$. Thus we must have

$$
\eta<(1+\eta) M h \quad \text { or } \quad \eta<M h /(1-M h)
$$

if $M h<1$. Now $a, b$ are, in our case, between 0 and 1 ; so that $h$ is finite, and therefore $\eta$ will be small if $M$ is small. In applying the result, we shall be able to suppose $\lambda, \mu$ to have finite ratios to $k^{2}$, where $k$ is an integer that is increased without limit. If two quantities $A, B$, depending on $\lambda, \mu$, are such that $A / B$ and $B / A$ do not, in absolute magnitude, 
increase beyond a fixed finite limit when $k$ is increased without limit; we shall say that $A, B$ are of the same order of magnitude, or that $B$ is of the same order as $A$, or that $B$ is comparable with $A$, or symbolically $A \| B$.

Thus $P, P^{\prime}, P^{\prime \prime}$ are, in general, of the same order of magnitude as $k^{2}$, but for special values of $x$ one or more of them may fall below that order, that is, the ratio $P / k^{2}$, for instance, may diminish without limit as $k$ increases, and it will do so, for example, if $\lambda, \mu$ depend on $k$ in such a way that $\lambda / \mu$ constantly $=T / S$ for the value of $x$ in question.

Since

$$
\delta Q=-\frac{5}{16}\left(P^{\prime} / P\right)^{2}+\frac{1}{4} P^{\prime \prime} / P
$$

and $M$ is the greatest value of $\left|P^{-\frac{1}{2}} \delta Q\right|$ it follows that $M \| 1 / k$ if $P \| k^{2}$ throughout the path of integration.

Hence, under the same restriction, $\eta$ will $\| 1 / k$ and the error $\delta f(b, a)$ will tend to vanish in comparison with $f(b, a)$ as $k$ increases (1) if the argument $\int_{a}^{b} d \rho$ is not less than $\int_{a}^{b}|d \rho|$, that is, if the sign of $d \rho$ is constant along the path,* and also (2) if the argument $\int_{a}^{b}\left|d_{\rho}\right|$ does not increase beyond a finite limit when $k$ is increased, in which case $\int_{a}^{b} d \rho$ is finite also, and the factor $\sinh \int_{a}^{b} P^{\frac{1}{2}} d x$ will be finite.

We are not generally entitled to use $f(b, a)$ as an approximation for $\phi(b, a)$

(1) if for some value of $x$ on the path $a b, P / k^{2}$ tends to zero as $k$ increases : for then $\delta Q$ may increase without limit with $k$;

(2) if $d \rho$ is not of constant sign on the path $a b$, and $\int_{a}^{b}|d \rho|$ increases indefinitely with $k$, for then the possible error

may much exceed the estimated value

$$
\eta\{P(b) P(a)\}^{-\frac{1}{2}} \cosh \int_{a}^{b}|d \rho|
$$

$$
\{P(b) P(a)\}^{-\frac{1}{t}} \sinh \int_{a}^{b} P^{\frac{1}{2}} d x .
$$

The last exception (2) can easily be avoided in the application we have

* Since $x$ is a real variable, this implies that the real part of $P^{\mathrm{l}}$ is of constant sign. It may be remarked, once for all, that, if $u$ is positive, the real part of $(u+w)+$ lies between $\sqrt{ } u$ and $\left(u^{2}+v^{2}\right)^{\ddagger}$, and the lateral part between $\frac{1}{2} v v / \sqrt{ } u$ and $\frac{1}{2} v\left(u^{2}+v^{2}\right)^{-1}$, and that, if $u$ is negative, the lateral part lies between $\sqrt{ } u$ and $\iota\left(u^{2}+v^{2}\right)^{\ddagger}$, the real part between $\mathfrak{v} v / \sqrt{ }(-u)$ and $t v\left(u^{2}+v^{2}\right)^{-!}$.

Neither part of $(u+v v)^{\frac{1}{2}}$ can vanish or change sign unless $v$ does so.

When $v$ is smull as compared to $u$, and $u$ is positive, the real part of $(u+v) t$ differs from $\sqrt{ } \mathfrak{u}$ by a quantity $<\left(u^{2}+v^{2}\right)^{\ddagger}-\sqrt{ } u$ and therefore $\| v^{2} / u^{\frac{3}{2}}$ at most. 
to make, but the restriction implied by (1) is a difficulty that must be removed.

Under the same restrictions, the derivatives of $f(b, a)$ furnish approximate values for those of $\phi(b, a)$. For instance,

$$
\delta f^{\prime}(b, a)=\phi^{\prime}(b, a)-f^{\prime}(b, a),
$$

and by differentiating (7) with respect to $b$, we have

But

$$
\delta f^{\prime}(b, a)=\int_{a}^{b} f^{\prime}(b, x)\{f(x, a)+\delta f(x, a)\} \delta Q d x .
$$

$f^{\prime}(b, a)=\{P(b) / P(a)\}^{\frac{1}{2}} \cosh \int_{a}^{b} P^{\mathfrak{k}} d x-\frac{1}{4} P^{\prime}(b)[P(a)]^{-\frac{1}{k}}[P(b)]^{-\frac{1}{4}} \sinh \int_{a}^{b} P^{\mathfrak{k}} d x$,

and $\left|f^{\prime}(b, a)\right| \ngtr|P(b) / P(a)|^{\frac{1}{2}} \cosh \int_{a}^{b}|d \rho|\left\{1+\frac{1}{4}\left|P^{\prime}(b)[P(b)]^{-\frac{1}{b}}\right|\right\}$.

Thus

$$
\begin{aligned}
\left|\delta f^{\prime}(b, a)\right| \ngtr \int_{a}^{b}|P(b) / P(x)|^{\frac{1}{2}} \cosh \int_{x}^{b}|d \rho|\left\{1+\frac{1}{\mid}\left|P^{\prime}(b)[P(b)]^{-\frac{1}{3}}\right|\right\} \\
\times(1+\eta)|P(x) P(a)|^{-\frac{1}{2}} \cosh \int_{a}^{x}|d \rho||\delta Q||d x| .
\end{aligned}
$$

or $|P(b) / P(a)|^{\frac{z}{z}}(1+\eta)\left\{1+\frac{1}{4} P^{\prime}(b)[P(b)]^{-\frac{\pi}{y}}\right\} \int_{a}^{b}\left|P^{-\frac{1}{2}} \delta Q\right| \cosh \int_{a}^{b}|d \rho||d x|$

or $\quad|P(b) / P(a)|^{z}\left\{1+\frac{1}{4} P^{\prime}(b)[P(b)]^{-i}\right\} \operatorname{Mh}(1+\eta) \cosh \int_{a}^{b}|d \rho|$.

Under the restrictions assumed, $P^{\prime}(b)[P(b)]^{-t}, M, \eta$ are $\| 1 / k$, so that $\delta f^{\prime}(b, a) \|$

$$
k^{-1}|P(b) / P(a)|^{\frac{1}{2}} \cosh \int_{a}^{b}|d \rho|
$$

that is, $1 / k$ of the estimated value $f^{\prime}(b, a)$.

Moreover, the second term in the value of $f^{\prime}(b, a)$ is only $\| \delta f^{\prime}(b, a)$, and thus we have, as stated in (6),

$$
\phi^{\prime}(b, a)=\{P(b) / P(a)\}^{\frac{1}{2}} \cosh \int_{a}^{b} P^{\frac{1}{a}} d x
$$

with an error \|

$$
k^{-1}|P(b) / P(a)|^{\frac{1}{2}} \cosh \int_{a}^{b} i d \rho \mid \text {. }
$$

The same method applies to $\phi^{\prime}(b, a)$ and $\phi^{\prime \prime}(b, a)$.

We shall apply the term "normal" to approximations in which the error is of the order of $1 / k$ of the estimated value or $1 / k^{2}$ of the derivative of the estimated value. The reference to the derivative is meant to save misunderstanding in the neighbourhood of a point where the function 
vanishes.* The values given under (6) are normal approximations under the restrictions assumed; they are, however, valid approximations under less narrow restrictions. The relative magnitude of the error, $\eta$, at most |l the greatest value of $\delta Q / P^{\star}$, that is,

$$
P^{\prime \prime} / 4 P^{\frac{8}{2}}-5 P^{\prime 2} / 16 P^{\frac{3}{2}} \quad \text { or } \quad-P^{-\frac{1}{d}} d^{2} / d x^{2}\left(P^{-\frac{1}{4}}\right),
$$

and, since $P^{\prime}, P^{\prime \prime}$ do not exceed the order of $k^{2}$, the error will be relatively small if $P^{\wedge}$ does not fall as low as the order $k^{4}$, that is; if $P$ is always. great in comparison with $k^{\frac{8}{5}}$. Also, when $d \rho$ is not of constant sign, the approximation is valid if $M \exp \int_{a}^{b}\left|d_{\rho}\right|$ is small, which will happen if the real part of $P$ is always negative and $\| k^{2}$, and the lateral part small in comparison with $k \log k$.

The results of $\$ \$ 8-14$ are not wanted till $\$ 22$, and the reader is advised to skip them for the time being. They relate to exceptional cases in which the formule of $\$ 7$ do not apply.

\section{Approximation by means of Riccati Functions.}

8. We must now seek for approximate values of $\phi$ and its derivatives when there is a value of $x$ on the path of integration at which $P / k^{x}$ diminishes without limit when $k$ increases. The case that is of importance is when the lateral part of $P$ is of a lower order than $k^{2}$ but of constant sign, while the real part is generally $\| k^{2}$ but occasionally zero. Thus the path of integration passes near a value of $x$ for which $P=0, \dagger$ and in such a neighbourhood the values (6) could. not well be approximations to $\phi(b, a), \ldots$, since they are not one-valued functions of $b$, on account of the surd factor, whereas $\phi(b, a)$ and its derivatives are one-valued functions of $b$.

When the zero of $P$ is simple and isolated + we may use, instead of the hyperbolic sine of the former approximation, the function $\omega$ which satisfies the equation

$$
\frac{d^{2} \omega}{d x^{2}}=x \omega
$$

This equation is included in Riccati's, and the functions satisfying it are as definite as the sine and cosine, though not so familiar. The notation $\omega(b, a)$, $\omega^{\prime}(b, a), \ldots$, will be used as in former cases.

- For instance, if the approximate value were $\cos k x$, with a possible error of $1 / k$, the approximation would be normal even when $x=\pi / 2 k$, although for that value the error would exceed the estimated value.

† If we write $P_{1},{ }_{2}$ for the parts of $P$ which are real and purely imaginary when $x$ is real. and suppose $P_{1}=0$ when $x=a$, then $P=0$ for a value of $x$ which is approximately

$$
a-\imath P_{2}(a) / P_{1}^{\prime}(a)
$$

and which tends to coincide with $a$ when $k$ is made great, since $P_{2}$ is, by supposition, of a lower order than $k^{2}$.

\$ That is, when no other zero tends to coincide with it as $k$ increases. 
Now, if, in (1), we change the independent variable from $x$ to $w$, a known function of $x$, the equation becomes

$$
\frac{d^{3}}{d w^{2}}\left(\phi \sqrt{ } w^{\prime}\right)=\phi \sqrt{ } w^{\prime}\left[P+\frac{1}{2}\{w, x\}\right] / w^{\prime 2},
$$

where $\{w, x\}$ is the Schwarzian derivative.

We choose $w$ so that $P / w^{\prime 2}=v$, that is, we take

$$
\frac{2}{3} w^{\frac{2}{2}}=\int P^{\dagger} d x \text {, }
$$

fixing the lower limit in this integral at the (complex) value of $x$ for which $P$ vanishes. Then $w$ is an analytical function of $x$ for values within a certain domain which includes the point where $P$ vanishes and which does not diminish indefinitely as $k$ increases. When $a$ is in the domain at a finite distance from the zero of $P, w \| k^{3}$. The domain includes the real value of $x$ for which the real part of $P$ vanishes, since the zero of $P$ tends to that value as a limit when $k$ increases.

We propose to prove that an approximate value of $\phi\left(x, x_{0}\right)$ is

$$
\left(w^{\prime} w_{0}^{\prime}\right)^{-\frac{1}{2}} \omega\left(w, w_{0}\right)
$$

and therefore substitute this expression for $f\left(x, x_{0}\right)$ in the Lemma (7). We must then have

$$
Q=f^{\prime \prime} / f=P-\frac{1}{2}\{w, x\}, \quad \delta Q=\frac{1}{2}\{w, x\}=w^{\prime \prime \prime} / 2 w^{\prime}-3 w^{\prime \prime 2} / 4 w^{\prime 3}, \quad Q+\delta Q=P ;
$$

so that $f+\delta f=\phi$.

In the domain $\delta Q$ is finite, however great $l$ may be, since we may exclude from the domain values of $x$ for which $w^{\prime}$ tends to zero as $k$ increases. To ascertain the degree of approximation, we must find the order of magnitude of $f(b, x) f(x, a) / f(b, a)$ by a discussion of the function $w$.

\section{Approximate Value of $\omega$.}

9. Now, for great values of the argument, we may approximate to $\omega$ by means of the functions $x$ which satisfy the equation

$$
\frac{d^{2} \chi}{d x^{2}}=\left(x+\frac{5}{16 x^{2}}\right) x
$$

and which are known, since we have, in fact,

$$
\chi(b, a)=a^{-\frac{1}{4}} b^{-\frac{1}{2}} \sinh \frac{2}{3}\left(b^{\frac{3}{2}}-a^{\frac{3}{2}}\right) .
$$

In the Lemma (7) we may put $\chi$ for $f, \omega$ for $f+\delta f$, and $-\frac{5}{16 \bar{x}^{2}}$ for $\delta Q$. Thus

$$
\omega(b, a)-\chi(b, a)=-\frac{5}{16} \int_{a}^{b} \chi(b, x) \omega(x, a) \frac{d x}{x^{2}} .
$$

Suppose now that, as $x$ travels from $a$ to $b$, the real part of $x^{\frac{3}{1}}$ does not ever decrease; then the real part of $x_{1}^{\frac{3}{3}}-x_{0}^{\frac{\pi}{2}}$ is positive or zero, and

$$
\left|\sinh \frac{2}{3}\left(x_{1}^{3}-x_{0}^{3}\right)\right| \ngtr\left|\exp \frac{2}{3}\left(x_{1}^{\frac{3}{3}}-x_{0}^{3}\right)\right|
$$

if $x_{0}, x_{1}$ are two valnes on the path $a b$ in the order stated. 
Suppose $\kappa$ to be the greatest value of

$$
\left|\omega\left(x_{1}, x_{0}\right)-\chi\left(x_{1}, x_{0}\right) i /\right| x_{1}^{-\frac{1}{2}} x_{0}^{-\frac{z}{2}} \exp \frac{2}{3}\left(x_{1}^{\frac{3}{2}}-x_{0}^{\frac{3}{3}}\right) \mid
$$

for all such positions of $x_{0}, x_{1}$ on the path.

Then we have, as in $\S 7$,

and thus

$$
|\omega(b, a)-\chi(b, a)| \ngtr \frac{5}{16} \mid a^{-\frac{1}{6}} b^{-7} \exp \frac{\frac{2}{3}}{3}\left(b^{\frac{3}{2}}-a^{\frac{3}{3}}\right)(1+\kappa) \int_{a}^{b} \cdot x^{-\frac{1}{2}} d x ;,
$$

$$
\kappa \ngtr \frac{5}{16}(1+\kappa) \int_{a}^{b} a-\frac{5}{2} d x \text { : }
$$

Now $\int_{a}^{b} \mid x^{-\frac{s}{2}} d x^{i}=\frac{2}{3}$ of the length of the path described by the point representing $x^{-\frac{1}{3}}$, and this will be of no higher order of magnitude than the greatest value of $\left|x^{-3}\right|$ on the path. Hence $\kappa$ is of the same order also when $x$ is great all along the path $a b$.

We conclude, therefore, that, if $a, b$ can be joined by a path along which $x$ is always great, and the real part of $x^{\frac{3}{2}}$ does not ever decrease, the difference

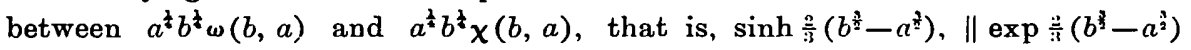
multiplied by the greatest value of $x^{-\frac{1}{2}}$ on the path.

As in $\S 7$, it follows that

$$
\begin{gathered}
a^{\frac{1}{4}} b^{-\frac{1}{2}} \omega^{\prime}(b, a)-\cosh \frac{2}{3}\left(b^{\frac{3}{2}}-a^{\frac{3}{2}}\right), \quad a^{-\frac{1}{2}} b^{\frac{1}{4}} \omega^{\prime}(b, a)+\cosh \frac{2}{3}\left(b^{\frac{3}{3}}-a^{\frac{3}{3}}\right), \\
a^{-\frac{1}{2}} b^{-\frac{1}{4}} \omega^{\prime \prime}(b, a)+\sinh \frac{2}{3}\left(b^{\frac{3}{2}}-a^{\frac{3}{2}}\right)
\end{gathered}
$$

are also of the same order of magnitude.

Approximations to $\omega(a, 0)$ and it. Derivatives when $a$ i.s Negative and lireat.

10. The approximations here given do not apply immediately to $\omega(a, 0)$ and its derivatives. In order to deal with these we notice first that they can be expanded directly from the differential equation in series of ascending powers of $a$, which are absolutely convergent for all finite values of $a$. Thus

$$
\begin{aligned}
& \omega(a, 0)=a+\frac{a^{4}}{3.4}+\frac{a^{7}}{3 \cdot 4 \cdot 6 \cdot 7}+\frac{a^{10}}{3 \cdot 4 \cdot 6 \cdot 7 \cdot 9 \cdot 10}+\ldots, \\
& \omega^{\prime}(a, 0)=1+\frac{a^{5}}{3}+\frac{a^{0}}{3.4 .6}+\ldots, \\
& -\omega^{\prime}(a, 0)=1+\frac{a^{3}}{2.3}+\frac{a^{0}}{2.3 .5 .6}+2.3 . \frac{a^{0}}{5.6 .8 .9}+\ldots \\
& -\omega^{\prime \prime}(a, 0)=\frac{a^{2}}{2}+\frac{a^{5}}{2 \cdot 3.5}+\ldots .
\end{aligned}
$$

When $a=-1$, in particular, these are all numerically less than 1 .

Again, if $\frac{d^{2} \omega}{d x^{2}}=x \omega$,

$$
\frac{d}{d x}\left\{\omega^{2}-\frac{1}{x}\left(\begin{array}{c}
d \omega \\
d x
\end{array}\right)^{2}\right\}=\frac{1}{x^{2}}\left(\frac{d \omega}{d x}\right)^{2}
$$


a positive quantity ; so that, if $x$ is negative, $\omega^{2}-\omega^{2 /} / x$ decreases as $-x$ increases. Hence, when $a<-1$,

$$
\{\omega(a, 0)\}^{2}-\left\{\omega^{\prime}(a, 0)\right\}^{2} / a<2, \quad\left\{\omega^{\prime}(a, 0)\right\}^{2}-\left\{\omega^{\prime}(a, 0)\right\}^{2} / a<2,
$$

since each of these $<2$ when $a=-1$.

Thus, since $a$ is negative,

$$
\omega(a, 0)<\sqrt{ } 2, \quad \omega(a, 0)<\sqrt{ } 2, \quad \omega^{\prime}(a, 0)<\sqrt{ }(-2 a), \quad \omega^{\prime \prime}(a, 0)<\sqrt{ }(-2 a) .
$$

Now the approximate values found for $\omega(b, a)$ and its derivatives may be used when $a, b$ are both negative, since, on the negative part of the real axis, the real part of $x^{\frac{\pi}{3}}$ is always 0 . Also, if $a$ is numerically less than $b$, the error $\| a^{-\frac{3}{3}}$, since $\frac{a}{3}\left(b^{\frac{1}{3}}-a^{\frac{\pi}{3}}\right)$, the argument of the exponential factor, is a pure imaginary.

We have then, as in $\S 2$,

$$
\omega(b, 0)=-\omega(a, 0) \omega^{\prime}(b, a)+\omega^{\prime}(a, 0) \omega(b, a)
$$

accurately, and approximately

$$
b^{\frac{1}{2}} \omega(b, 0)=x^{\frac{1}{4}} \omega(a, 0) \cosh \frac{\frac{a}{3}}{3}\left(b^{\frac{3}{2}}-a^{\frac{3}{2}}\right)+a^{-\frac{3}{2}} \omega^{\prime}(a, 0) \sinh \frac{\frac{2}{3}}{3}\left(b^{\frac{b}{b}}-a^{\frac{3}{4}}\right) .
$$

Since $\omega(a, 0)<\sqrt{ } 2, \omega^{\prime}(a, 0)<\sqrt{ }(-2 a)$, and the hyperbolic factor is subject to a possible error $\| a^{-\frac{3}{2}}$, the whole expression is subject to an error $\| a^{-\frac{5}{4}}$ at most.

Also, since we hare

$$
\omega^{\prime}(b, 0)=-\omega(a, 0) \omega^{\prime \prime}(b, a)+\omega^{\prime}(a, 0) \omega^{\prime}(b, a),
$$

$$
b^{-\frac{1}{2}} \omega^{\prime}(l, 0)=a^{\frac{3}{4}} \omega(a, 0) \sinh \frac{\frac{2}{3}}{3}\left(b^{\frac{2}{2}}-a^{\frac{3}{2}}\right)+a^{-\frac{1}{4}} \omega^{\prime}(a, 0) \cosh \frac{2}{3}\left(b^{\frac{3}{2}}-a^{\frac{3}{3}}\right),
$$

with a possible crror of the same order again. From (10), (11) we have

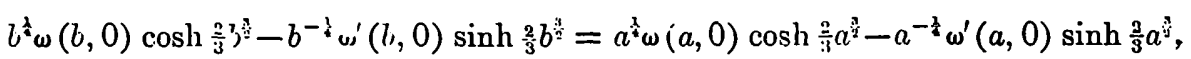
with a possible error $\| a^{-t}$ at most. This is true when $a, b$ are real and negative. and $b$ is numerically greater than $a$.

Hence, as $n$ travels along the real axis towards $-\infty$,

$$
a^{\frac{1}{2}} \omega(a, 0) \cosh \frac{2}{3} a^{\frac{1}{2}}-a^{-\frac{1}{2}} \omega^{\prime}(a, 0) \sinh \frac{2}{3} c^{\frac{\pi}{2}}
$$

tends to a finite limiting value, say $A_{\mathfrak{1}}$.

In the same way

$b^{\frac{1}{2}} \omega(b, 0) \sinh \frac{2}{3} b^{\frac{3}{2}}-b^{-\frac{1}{4}} \omega^{\prime}(b, 0) \cosh \frac{2}{3} b^{3}=a^{\frac{1}{2}} \omega(a, 0) \sinh \frac{2}{3} a^{3}-a^{-\frac{1}{4}} \omega^{\prime}(a, 0) \cosh \frac{2}{3} a^{\frac{3}{2}}$, with a possible error $\| a^{-\hat{4}}$, and the last expression also tends to a finite limit, say $A_{2}$, as $a$ travels towards $-\infty$ on the real axis.

Hence

$$
\begin{aligned}
a^{\frac{1}{4}} \omega(a, 0) & =A_{1} \cosh \frac{2}{3} a^{\frac{3}{2}}-A_{9} \sinh \frac{2}{3} a^{\frac{3}{2},} \\
a^{-\frac{1}{2}} \omega^{\prime}(a, 0) & =\ddot{A_{1}} \sinh \frac{2}{3} a^{\frac{3}{2}}-A_{2} \cosh \frac{2}{3} a^{\frac{3}{4}},
\end{aligned}
$$

and

with an error in each case $\| a^{-\frac{n}{4}}$ at most. In forming this estimate of the error, we used the inequalities $\omega(a, 0)<\sqrt{ } 2, \omega^{\prime}(a, 0)<\sqrt{ }(-2 a)$; but, as it now appears that $a^{\frac{3}{a}} \omega(a, 0)$ and $a^{-\frac{1}{2}} \omega^{\prime}(a, 0)$ do not exceed certain finite limits, howerer great - $a$ may be, we may say that the errors are $\| a^{-\frac{1}{2}}$ at most. 


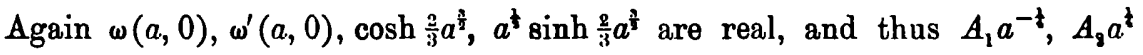
are real. We may therefore put

$$
A_{1}=A \sinh \alpha, \quad A_{2}=-A \cosh a,
$$

where $a$ is a pure imaginary and $A$ a complex quantity whose amplitude (vectorial angle) is $-\frac{1}{4} \pi$ or $\frac{3}{4} \pi$, that of $a$ being $\pi$.

Thus

$$
\omega(a, 0)=A a^{-\frac{1}{t}} \sinh \left(\frac{3}{i} a^{\frac{3}{2}}+a\right), \quad \omega^{\prime}(a, 0)=A a^{\frac{3}{3}} \cosh \left(\frac{2}{s} a^{4}+a\right)
$$

nearly. In the same way, starting with the accurate equation,

$$
\omega^{\prime}(b, 0)=-\omega^{\prime}(a, 0) \omega^{\prime}(b, a)+\omega^{\prime \prime}(a, 0) \bullet(b, a),
$$

we may prove the approximate formulø

$$
a^{\frac{\xi}{\omega}} \omega^{\prime}(a, 0)=B \sinh \left(\frac{2}{3} a^{\frac{3}{2}}+\beta\right), \quad a^{-\frac{k}{4} \omega^{n}}(a, 0)=B \cosh \left(\frac{2}{3} a^{\frac{3}{2}}+\beta\right),
$$

where the error $\| a^{-\frac{\beta}{2}}$ in each case, $\beta$ is a pure imaginary, and $B$ a complex quantity of amplitude $-\frac{1}{4} \pi$ or $\frac{3}{4} \pi$.

The same when the Vectorial Angle of a is between $\frac{1}{3} \pi$ and $\frac{5}{3} \pi$.

11. These expressions have been found on the supposition that $a$ is real, negative, and great. They may be extended by the use of the formulæ for $\omega(b, a)$ and its derivatives. Since the vectorial angle of $a$ is $\pi$, that of $a^{\frac{3}{3}}$ is $\frac{3}{3} \pi$; so that $a^{\frac{3}{3}}$ lies on the lower part of the imaginary axis. If, then, along the path $a b$ the real part of $x^{\frac{3}{2}}$ constantly increases, $b^{\frac{3}{3}}$ must lie in the fourth or fifth quadrant, and the vectorial angle of $b$ must lie between $\pi$ and $\frac{5}{3} \pi$. For such $a$ value of $b$ we may use the approximate valnes of $\omega(b, a), \ldots$, but the smallness of the error depends on our being able to keep the path $a b$ well away from the origin, since the order of the possible error is that of the least value of $\left|x^{-\frac{1}{2}}\right|$ on the path. Hence the approximation would not be valid if the vectorial angle of $b$ were actually $\frac{5}{3} \pi$.

The approximate formulæ give

$$
\begin{aligned}
\omega(b, 0)= & -\dot{\omega}(a, 0) \omega^{\prime}(b, a)+\omega^{\prime}(a, 0) \omega(b, a) \\
= & A a^{-\frac{1}{2}} \sinh \left(\frac{2}{3} a^{\frac{3}{3}}+a\right) a^{\frac{4}{3}} b^{-\frac{1}{2}} \cosh \frac{2}{3}\left(b^{\frac{3}{3}}-a^{\frac{3}{2}}\right) \\
& \quad+A a^{\frac{3}{4}} \cosh \left(\frac{2}{3} a^{\frac{3}{2}}+a\right) a^{-\frac{1}{2}} b^{-\frac{1}{2}} \sinh \frac{2}{3}\left(b^{\frac{3}{2}}-a^{\frac{3}{3}}\right) \\
= & A b^{-\frac{1}{2}} \sinh \left(\frac{2}{3} b^{\frac{3}{3}}+a\right),
\end{aligned}
$$

with a possible error $\| x_{0}^{-\frac{1}{2}} b^{-\frac{1}{4}} \cosh \frac{2}{3}\left(b^{\frac{3}{3}}-a^{\frac{3}{3}}\right)$, where by $x_{0}$ we are to understand the least absolute value taken by $x$ on the path $a b$. Now $a$ may be as great numerically as we please, and, if we restrict $b$ to have an amplitude between $\pi$ and $\frac{4}{3} \pi, b^{\frac{3}{2}}$ will lie in the fourth quadrant, and the path $a b$ may be so drawn that $|x|$ is never less than $|b|$, so that we have $x_{0}=|b|$.

Hence, as $a^{\frac{3}{3}}$ is a pure imaginary, the possible error in the value of $\omega(b, 0)$

$$
\| b^{-\frac{1}{4}} \exp \frac{2}{3} b^{\frac{4}{4}}
$$

SHR. 2. VOL. 5 . NO. 971 . 
Similarly $\quad \omega^{\prime}(b, 0)=A b^{\frac{1}{t}} \sinh \left(\frac{2}{3} b^{\frac{1}{4}}+a\right)$,

with a possible error $\| b^{-t} \exp \frac{2}{3} b^{\ddagger}$.

To the same respective degrees of approximation

$$
\omega^{\prime}(b, 0)=B b^{-\frac{1}{4}} \sinh \left(\frac{2}{3} b^{\xi}+\beta\right), \quad \omega^{\prime \prime}(b, 0)=B b^{\frac{1}{2}} \cosh \left(\frac{3}{3} b^{\xi}+\beta\right) .
$$

The amplitude of $b$ has been restricted to values greater than $\pi$ and less than $\frac{6}{3} \pi$, but it is easily seen that it may be greater than $\frac{4}{3} \pi$ so long as it does not approach $\frac{5}{3} \pi$. Also, if it is less than $\pi$ but greater than $\frac{1}{3} \pi$, a path $a b$ can be drawn along which one of the two values of $x^{3}$ has an increasing real part, and the expressions given still hold. We may say that the errors are $\|$

$$
b^{-\frac{3}{2}} \exp \frac{2}{3} \sqrt{ } b^{3} \text { and } b^{-\frac{4}{5}} \exp \frac{2}{3} \sqrt{ } b^{3},
$$

using $\sqrt{ } b^{3}$ for that value which has a positive real part, while we keep the fractional power, such as $b^{4}$, to denote the value whose amplitude is $\frac{3}{2}$ of that of $b$.

The same for other great values of $a$. Values of $A B, a, \beta$.

12. It is now easy to find approximate values for $\omega(a, 0)$ and its derivatives when the vectorial angle of $a$ takes any value whatever, as, for instance, a value between $\pm \frac{1}{3} \pi$.

The expressions for $\omega(a, 0), \ldots$ as series shew that

$$
\begin{aligned}
\omega\left(a e^{3 l \pi}, 0\right) & =e^{3 l \pi} \omega(a, 0), \\
\omega^{\prime}\left(a e^{3 ! \pi}, 0\right) & =\omega^{\prime}(a, 0), \\
\omega^{\prime}\left(a e^{3 l \pi}, 0\right) & =\omega^{\prime}(a, 0) \\
\omega^{\prime \prime}\left(a e^{3 l t \pi}, 0\right) & =e^{-34 \pi} \omega(a, 0) .
\end{aligned}
$$

When the amplitude of $a$ is between 0 and $\frac{1}{3} \pi$, that of $a e^{3 / \pi}$ is between $\frac{3}{3} \pi$ and $\pi$, and we may therefore use the known formulæ for $\omega\left(a e^{3 \mathrm{k} \pi}, 0\right)$.

Thus

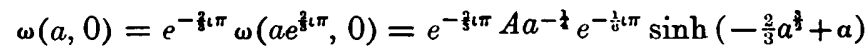

$$
\begin{aligned}
& =A e^{-\frac{5}{b} t \pi} a^{-\frac{t}{4}} \sinh \left(-\frac{2}{3} a^{\frac{4}{4}}+a\right)
\end{aligned}
$$

approximately. So also

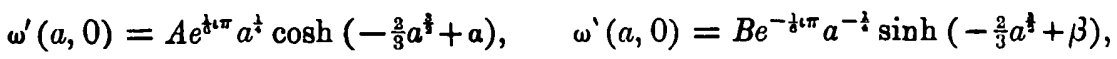

$$
\begin{aligned}
& \omega^{\prime \prime}(a, 0)=B e^{\frac{8}{2} t \pi} a^{\frac{1}{6}} \cosh \left(-\frac{2}{3} a^{\frac{3}{3}}+\beta\right),
\end{aligned}
$$

nearly, the possible errors being as before.

The values of $a, \beta$ can now be found from the consideration that $\omega(a, 0)$ and its derivatives are real when $a$ is real and positive, as follows at once from the expansions in powers of $a$. As $a$ increases, $\sinh \left(-\frac{2}{3} a^{4}+a\right)$ tends to equality with $-\frac{1}{2} \exp \left(\frac{2}{3} a^{\xi}-a\right)$, so that its vectorial angle is $c u$ in the limit, and $A e^{-\frac{5}{2} t \pi-a}$ must be real. Sir 00 vectorial angle of $A$ is $-\frac{1}{4} \pi$, we must have

$$
-\frac{1}{4} \iota \pi-\frac{8}{6} \iota \pi-a=a \text { multiple of } \iota \pi,
$$

so that we may take $a=-\frac{1}{1} \frac{1}{\varepsilon} \iota \pi$, say. In the same way we may take $\beta=-\frac{3}{1 \frac{3}{2}} \iota \pi$. 
Again $\quad \mathrm{l}=\omega(a, 0) \omega^{\prime \prime}(a, 0)-\omega^{\prime}(a, 0) \omega^{\prime}(a, 0)=A B \sinh (a-\beta)$,

so that

$$
A B=\operatorname{cosech} \frac{1}{3} \iota \pi=-2 \iota / \sqrt{ } 3 .
$$

Since the amplitude of $A$ is $-\frac{1}{4} \pi$ or $\frac{3}{4} \pi$ and that of $A B$ is $-\frac{1}{2} \pi$, it follows that $B / A$ is positive.

When the amplitude of $a$ is between 0 and $-1 \pi$ similar expressions can be found. The same expressions may, in fact, be used for amplitudes between $\pm \frac{1}{3} \pi$, exclissive; we apparently get different results by deriving the functions of $a$ from those of $a e^{j / \pi}$ and $a e^{-j i \pi}$, but the difference disappears if we use the formulæe

$$
-\sinh \left(-\frac{2}{3} a^{\frac{3}{2}}+a\right)=\cosh \left(-\frac{2}{3} a^{\frac{3}{2}}+\alpha\right)=\frac{1}{2} \exp \left(\frac{2}{3} a^{\frac{3}{3}}-a\right),
$$

which hold approximately when $a$ is great and has an amplitude between $\pm \frac{1}{3} \pi$, exclusive.

\section{The Function $\chi$.}

13. As $a$ tends to $+\infty, \omega(a, 0) / \omega^{\prime}(a, 0)$ tends to the value $-A / B$. Let $\chi(a)$ be written for $\omega(a, 0)+A / B \omega^{\prime}(a, 0)$; then $\chi, \chi^{\prime}$ cannot vanish except for real negative values of the argument, though they tend to zero when the argument is positive and increasing. For we have

$$
\frac{d}{d x} \frac{\omega^{\prime}(x, 0)}{\omega(x, 0)}=\{\omega(x, 0)\}^{-2}=\frac{4}{A^{2}} \cdot x^{\frac{3}{3}} e^{-3} x^{\frac{3}{2}}
$$

nearly, and, by integration between $a$ and $+\infty$,

$$
\frac{\omega^{\prime}(a, 0)}{\omega(a, 0)}+\frac{B}{A}=\frac{2 \iota}{A^{2}} e^{-\mathbf{s}^{\frac{3}{c^{2}}}}
$$

if $a$ is a great quantity of amplitude between $\pm \frac{1}{3} \pi$. Thus

$$
\chi(a)=\frac{A}{B} \omega(a, 0) \underset{A^{2}}{2 \iota} e^{-\frac{1}{a^{\frac{3}{2}}}},
$$

which tends to zero as the real part of $a^{\frac{3}{3}}$ increases, being $\| a^{-\frac{1}{4}} e^{-3 a t}$.

Similarly

$$
\frac{d}{d x} \frac{\omega^{\prime \prime}(x, 0)}{\omega^{\prime}(x, 0)}=-x\left\{\omega^{\prime}(x, 0)\right\}^{-2},
$$

from which it follows that $\chi^{\prime}(a)$ also tends to zero.

As $x$ increases from $0, \omega^{\prime}(x, 0) / \omega(x, 0)$ continually increases, beginning at $-\infty$, while $\omega^{\prime \prime}(x, 0) / \omega^{\prime}(x, 0)$ continually decreases; beginning at 0 . Thus $\chi(x)$, $\boldsymbol{X}^{\prime}(x)$ can never vanish for positive values of $x$. Moreover,

$$
\chi(x+\iota y) \chi^{\prime}(x-\iota y)-\chi(x-\imath y) \chi^{\prime}(x+\iota y)=2 \iota y \int_{x}^{\infty} \chi(x+\iota y) \chi(x-\iota y) d x
$$

from which it follows in the usual way that $\chi(x+\imath y)$ or $\chi^{\prime}(x+\imath y)$ can only vanish when $y=0$. It is therefore proved that $\chi, x^{\prime}$ can only vanish for real negative values of the argument. 
Approximation to $\omega(b, a)$.

14. We can now find approximate values for $\omega(b, a)$ and its derivatives whatever the amplitudes of $a, b$, provided the absolute values of $a, b$ are great. Take, for instance, the amplitude of $b$ to be small and that of $a$ nearly equal to $\pi$ : to fix the ideas, let these amplitudes be between $\pm \frac{1}{6} \pi$ and $\pi \pm \frac{1}{6} \pi$ respectively. These limits are amply wide enough for the application that is to be made of the results; if they were not, somewhat wider ones could be taken without affecting the argument.

Now the real part of $b^{\frac{\pi}{2}}$ is great, and thus

$$
\sinh \left(\frac{\ddot{3}}{3} b^{\frac{\pi}{2}}-\alpha\right), \quad \cosh \left(\frac{\frac{2}{3}}{3} b^{\frac{3}{2}}-a\right), \quad \frac{1}{2} \exp \left(\frac{\ddot{3}}{3} b^{\frac{3}{2}}-a\right)
$$

are practically the same, the differences being inconsiderable even in comparison with $b^{-\frac{3}{2}}$. The same is true when the argument is $\frac{2}{3} b^{\frac{1}{2}}-j$.

Also, we have

$$
\omega(b, a)=\omega(a, 0) \omega^{\prime}(b, 0)-\omega(b, 0) \omega^{\prime}(a, 0),
$$

the approximate value of which is

$$
\begin{aligned}
& A a^{-\frac{1}{3}} \sinh \left(\frac{\frac{3}{3}}{3} a^{\frac{3}{2}}+a\right) \frac{1}{2} B e^{\frac{8}{4} t \pi} b^{-\frac{1}{4}} \exp \left(\frac{\frac{1}{3}}{3} b^{\frac{3}{2}}-\beta\right) \\
& -\frac{1}{2} d e^{\frac{1}{5} \iota \pi} b^{-\frac{1}{4}} \exp \left(\frac{\frac{3}{3}}{3} b^{\frac{3}{2}}-a\right) B a^{-\frac{1}{4}} \sinh \left(\frac{2}{3} a^{\frac{1}{2}}+\beta\right)
\end{aligned}
$$

(1r

$$
\frac{1}{9} A B a^{-\frac{1}{3}} b^{-\frac{1}{4}}\left\{\sinh \left(\frac{2}{3} a^{\frac{3}{2}}-\frac{\iota \pi}{12}\right) \exp \left(\frac{2}{3} b^{\frac{3}{2}}+\frac{5}{4} \iota \pi\right)-\sinh \left(\frac{2}{3} a^{\frac{3}{2}}-\frac{5 \iota \pi}{12}\right) \exp \left(\frac{2}{3} b^{\frac{3}{2}}+\frac{\iota \pi}{4}\right)\right\}
$$

or, on simplifying and substituting the value of $A B$,

$$
a^{-\frac{1}{4}} b^{-\frac{1}{4}} \exp \left(\frac{2}{3} l^{\frac{3}{2}}+\frac{8}{4} \iota \pi\right) \sinh \left(\frac{2}{3} a^{\frac{3}{2}}-\frac{\iota \pi}{4}\right) .
$$

l:or this, again, we may write

$$
a^{-\frac{1}{2}} b^{-\frac{1}{2}}\left\{c \sinh \frac{2}{3}\left(b^{\frac{3}{2}}+a^{\frac{3}{2}}\right)+\sinh \frac{2}{3}\left(b^{\frac{\pi}{2}}-a^{\frac{3}{2}}\right)\right\}
$$

so long as the real parts of $b^{\frac{3}{2}} \pm a^{\frac{3}{2}}$ are both positive and great; this is really no restriction, for, when $a$ is negative, $a^{\dot{3}}$ is purely imaginary and the condition is certainly fulfilled. When the amplitude of $a$ is below $\pi$, the real part of $a^{\frac{\pi}{4}}$ is negative, and in $b^{\frac{3}{2}}+a^{\frac{4}{2}}$ it is possible that the real part may not be great or may even be negative; but, if this happened, the first term $\iota \sinh \frac{2}{3}\left(b^{\frac{3}{3}}+a^{\frac{3}{3}}\right)$ would be inconsiderable in comparison with the second $\sinh \frac{2}{3}\left(b^{\frac{7}{5}}-a^{\frac{3}{5}}\right)$. The converse would happen if the amplitude of $a$ were above $\pi$.

The second term $a^{-\frac{1}{4}} b^{-\frac{2}{4}} \sinh \frac{3}{3}\left(b^{\frac{3}{3}}-a^{\frac{3}{3}}\right)$ in the expression for $\omega(b, a)$ is the same in form as the original expression which we found by supposing $a, b$ negative. The other term $c a^{-\frac{1}{4}} b^{-\frac{1}{4}} \sinh \frac{2}{3}\left(b^{\frac{3}{2}}+a^{\frac{3}{2}}\right)$ can be derived from the same expression by putting $b e^{2 \text { ir }}$ for $b$, that is, by travelling from $a$ to $b$ through the third and fourth quadrants instead of through the second and first. Hence the result may be stated in the form of a rule; take the two paths from $a$ to $b$ one above the origin, the other below, apply the formula $a^{-\frac{1}{4}} b^{-\frac{1}{2}} \sinh \frac{2}{3}\left(b^{\frac{3}{2}}-a^{\frac{3}{3}}\right)$ to each of the two paths and add the results: the sum is the approximate value 
for $\omega(b, a)$. There is a similar rule, similarly proved, for the derivatives of $\omega(b, a)$. The expressions are

$$
\begin{aligned}
& \omega^{\prime}(b, a)=a^{-\frac{1}{4}} b^{\frac{3}{4}}\left\{c \cosh \frac{2}{3}\left(b^{\frac{3}{2}}+a^{\frac{3}{2}}\right)+\cosh \frac{2}{3}\left(b^{\frac{3}{2}}-a^{\frac{3}{2}}\right)\right\}, \\
& \omega^{\prime}(b, a)=a^{\frac{1}{4}} b^{-\frac{1}{4}}\left\{c \cosh \cdot_{3}^{2}\left(b^{\frac{\pi}{2}}+a^{\overline{2}}\right)-\cosh \frac{2}{3}\left(b^{\frac{3}{2}}-a^{\frac{3}{2}}\right)\right\}, \\
& \omega^{\prime \prime}(b, a)=a^{\frac{1}{4}} b^{\frac{1}{4}}\left\{\iota \sinh \frac{2}{3}\left(b^{\frac{3}{2}}+a^{\frac{5}{2}}\right)-\sinh \frac{2}{3}\left(b^{\frac{3}{2}}-a^{\frac{3}{5}}\right)\right\} \text {. }
\end{aligned}
$$

It is found without difficulty that, when $a, b$ are both $\| k^{2}$, these approximations are normal in the sense of $\S 7$.

When $a$ or $b$ is of a lower order of magnitude than $k^{\frac{2}{3}}$, the approximations are not so good, and when either $a$ or $b$ has a finite value, not increasing indefinitely with $k$, the error is of the same order of magnitude as the estimated value, so that we can ouly say that the estimated value is of the right order of magnitude. We can truly say so much in general, for, when $a$ is finite, $\omega(a, 0)$ and its derivatives are finite, and the hyperbolic functions of $\frac{2}{3}\left(b^{\frac{3}{3}} \pm a^{\frac{7}{2}}\right)$ are $\|$ those of $\frac{2}{3} b^{\frac{3}{2}}$; also the formula

$$
\omega(b, a)=\omega(a, 0) \omega^{\prime}(b, 0)-\omega^{\prime}(a, 0) \omega(b, 0)
$$

shews that $\omega(b, a)$, in general, $\| \omega^{\prime}(b, 0)$ and $\omega(b, 0)$. It must be added that the factor $a^{ \pm \frac{1}{4}}$ or $b^{ \pm \frac{1}{4}}$ is to be dropped when $a$ or $b$ approaches 0 , say when $|a|$ or $|b|<1$.

\section{Application to $\phi$.}

15. We are to apply these results to the problem of finding an approximate value for $\phi(b, a)$ and, in fact, finding the possibility of error when $f(b, a)$ is put for $\phi(b, a)$, where $f\left(x, x_{0}\right)=\left(w^{\prime} w_{0}^{\prime}\right)^{-4} w\left(w, w_{0}\right)$ as in $\S 8$.

Now $\frac{2}{3} w^{\frac{3}{3}}=\int P^{\frac{1}{d}} d x$, vanishing with $P$, and the lateral part of $P$ is of constant sign, say $+\iota$, but of lower order than $k^{2}$, while the real part vanishes and changes sign at a point of the path of $x$, but is generally of the order of $k^{*}$ : suppose the change to be from - to + as $x$ increases. Before the change the amplitude of $P^{\text {t }}$ is slightly less than $\frac{1}{2} \pi$; after the change it is slightly greater than 0 . In both parts of the path the real part of $w^{\frac{3}{3}}$ increases with $x$ and, in fact, the point $w^{\frac{3}{4}}$ comes up to the neighbourhood of the origin along a path in the third quadrant which lies near the imaginary axis, then crosses the real axis, describes a small arc in the second quadrant, and comes out into the first quadrant, where it follows a path slightly above the real axis. The point $w$ describes a path slightly above the real axis which begins far on the left in the second quadrant and ends far on the right in the first. We have found approximate values for $\omega(b, a)$ and its derivatives when $a b$ is such a path, and we can also approximate to $\omega(b, c), \omega(c, a)$ when $c$ is any point on the path.

We have

$$
f\left(x, x_{0}\right)=\left(w^{\prime} w_{0}^{\prime}\right)^{-\frac{1}{2}} \omega\left(w, w_{0}\right)=\left(w^{\prime} w_{0}^{\prime}\right)^{-\frac{1}{2}} \Sigma\left(w w_{0}\right)^{-\frac{1}{4}} \sinh \frac{2}{3}\left(w^{\frac{3}{2}}-w_{11}^{3}\right)
$$

nearly, the $\Sigma$ referring to the two possible paths from $w_{0}$ to $w$.

[The notation has been changed, $v_{0}, w$ taking the place of $a, b$, and $x_{0}, x$ being the corresponding values of the original variable $x$ in (1)]. 
But $w^{\prime 2} w=P, \frac{2}{3} u t^{\frac{1}{2}}=\int P^{\frac{1}{3}} d x$, so that

$$
f\left(x, x_{0}\right)=\Sigma\left(P P_{0}\right)^{-1} \sinh \int_{x_{0}}^{x} P^{\mathfrak{t}} d x \text { nearly. }
$$

Let $\eta$ be the greatest absolute value of

$$
\delta f\left(x_{1}, x_{2}\right) /\left(P_{1} P_{2}\right)^{-1} \exp \int_{x_{2}}^{x_{1}} P^{\mathbf{i}} d x
$$

for any positions of $x_{1}, x_{2}$ on the path $x_{0} x$, such that $x_{v}, x_{2}, x_{1}, x$ occur in this order. Also, let $\gamma$ be the greatest absolute value of

$$
f\left(x_{1}, x_{2}\right) /\left(P_{1} P_{2}\right)^{-1} \exp \int_{r_{2}}^{x_{1}} P^{\mathrm{l}} d x .
$$

Then we know that $\gamma$ does not exceed a fixed finite limit, however great $k$ nay be, since there is a finite limit to the absolute value of

$$
\omega\left(v_{1}, w_{2}\right) /\left(w_{1} w_{2}\right)^{-\frac{1}{4}} \exp \frac{2}{3}\left(w_{1}^{\frac{2}{2}}-v_{2}^{\frac{4}{4}}\right)
$$

whether $w_{1}, w_{2}$ are small, finite, or great, provided that the real part of $w^{\frac{3}{3}}$ constantly increases along the path $w_{2} w_{1}$.

Now $\phi=f+\delta f$, and thus $\left|\phi\left(x_{1}, x_{2}\right)\right|$ is not greater than

By the Lemma (7),

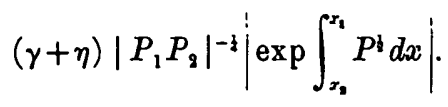

$$
\begin{aligned}
\left|\delta f\left(x, x_{0}\right)\right| & =\left|\int_{x_{0}}^{x} f\left(x, x_{1}\right) \phi\left(x_{1}, x_{0}\right) \delta Q_{1} d x_{1}\right| \\
& \leqslant \int_{x_{0}}^{x} \gamma(\gamma+\eta)\left|P P_{1}^{2} P_{0}\right|^{-\frac{1}{2}}\left|\exp \int_{x_{1}}^{x} P^{\mathbf{i}} d x\right|\left|\exp \int_{x_{0}}^{x_{1}} P^{\mathbf{l}} d x\right|\left|\delta Q_{1} d x_{1}\right|,
\end{aligned}
$$

and in this any two points on the path may be put for $x_{0}, x$, so that

$$
\eta \leqslant \gamma(\gamma+\eta) \int_{x_{0}}^{x}\left|P^{-i} \delta Q d x\right| .
$$

Now $\delta Q$ is finite; suppose $m$ to be its greatest value. $P^{-k} d x=2 d\left(P^{i}\right)_{/}^{\prime} P^{\prime}$, and $P^{\prime}$ always $\| k^{2}$; suppose $\left|P^{\prime}\right|$ always $>p k^{2}$, where $p$ is a fixed finite number. Thus

$$
\eta \leqslant 2 \gamma(\gamma+\eta) \frac{m}{p k^{2}} \int_{x_{0}}^{x_{1}}\left|d\left(P^{\natural}\right)\right|,
$$

and the last factor is the length of path described by the point $P^{\sharp}$, which $\| k$ and $<k q$, say, where $q$ is finite.

Thus

$$
\eta<2 \gamma(\gamma+\eta) m q / p k \text {. }
$$

Hence $\eta \| 1 / k$, that is, $f\left(x, x_{0}\right)$ is a normal approximation to $\phi\left(x, x_{0}\right)$. that

Since our approximation to $\omega(b, a)$ was normal when $a, b$ were $\| k^{k}$, it follows

$$
\left(P P_{0}\right)^{-\ddagger} \Sigma \sinh \int_{x_{0}}^{x} P^{t} d x
$$

is also a normal approximation to $\phi\left(x, x_{0}\right)$ unless, as $k$ increases, $x$ or $x_{0}$ tends 
to the value where $P=0$. Similar results hold for the derivatives, only differing in form from those of $\$ 7$ in the presence of $\Sigma$.

\section{Case when $P$ Vanishes near a Terminus.}

16. When the path $x_{0} x$ begins or ends in the neighbourhood of the point where $P=0$, the Riccati function $\omega$ still gives a normal approximation, but the hyperbolic function has a greater possibility of error, and, in fact, it gives a wrong order of magnitude when $v$ or $u_{0}$ is small.

Since $w^{\prime 2} w=P$, a finite value of $w$ corresponds to a value of $P$ of the order of $k^{\mathbf{s}}$; when $P$ is of a lower order than this, $w$ is small and the factor $10^{-1}$ is to be dropped in the expression for $\omega\left(w, w_{0}\right)$. Hence, when $|P|$ is less than $k^{\mathbf{3}}$, we are to substitute $k^{-t}$ for $P^{-\frac{1}{t}}$ in the expressions for $\phi\left(x, x_{n}\right), \phi^{\prime}\left(x, x_{n}\right)$. The results will give the true order of magnitude, bat will not be normal approximations. Similarly, in the expressions for $\phi^{\prime}\left(x, x_{0}\right), \phi^{\prime \prime}\left(x, x_{0}\right)$, we must substitute $k^{\frac{1}{3}}$ for $P^{\frac{1}{4}}$. When $|P| \geqslant k^{\mathrm{s}}$ these substitutions are not to be made, and the ordinary expressions give the true order of magnitude.

\section{Case when P. Vanishes more than Once.}

17. We may combine the results for $\phi$ and its derivatives in the statement that the value of the matrix $W\left(x, x_{0}\right)(\S 2)$ is nearly $\Sigma Y\left(x, x_{0}\right)$, where

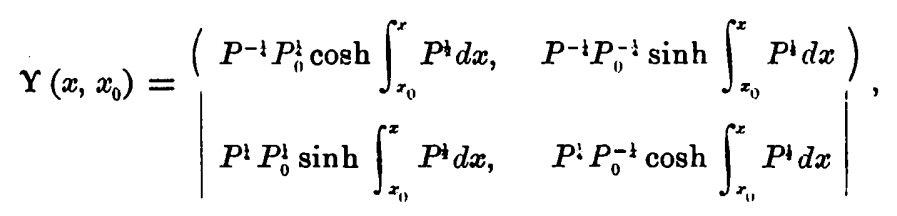

the approximation being normal except as noted. It is readily verified that

$$
\Upsilon\left(x_{1}, x\right) \Upsilon\left(x, x_{0}\right)=\Upsilon\left(x_{1}, x_{0}\right),
$$

and that this product is again a normal approximation to $W\left(x_{1}, x\right) W\left(x, x_{0}\right)$, or $W\left(x_{1}, x_{0}\right)$, if the real part of $P^{\mathbf{t}} d x$ has a constant sign along the whole path $x_{0} x x_{1}$, and if the factors in the one product are normal approximations to those in the other.

We also have, since the multiplication of matrices is distributive,

$$
\operatorname{\Sigma Y}\left(x_{1}, x\right) \mathbf{\Sigma Y}\left(x, x_{0}\right)=\operatorname{\Sigma Y}\left(x_{1}, x_{0}\right)
$$

where, if the first summation refers to a paths from $x$ to $x_{1}$, and the second to $\beta$ paths from $x_{0}$ to $x$, the third refers to the $a \beta$ combined paths $x_{0} x x_{1}$.

Hence, when on the path $a b$ the real part of $P$ vanishes (simply) at several points, say three, $c_{1}, c_{8}, c_{8}$ in order, we have

$$
W(b, a)=W\left(b, \frac{c_{2}+c_{3}}{2}\right) W\left(\frac{c_{8}+c_{3}}{2}, \frac{c_{1}+c_{9}}{2}\right) W\left(\frac{c_{1}+c_{9}}{2}, a\right) .
$$

Each factor refers to a part of the path in which there is only one of the points $c_{1}, c_{2}, c_{3}$, so that the approximation by means of the Riccati function can be used. 
The result is $\quad W(b, a)=\Sigma \Upsilon(b, a)$, nearly,

where the summation refers to the eight paths which are possible when one may go either above or below eacl one of the zeros of $P$. The approximation is normal unless $a$ or $b$ tends to a zero of $P$ when $k$ increases.

\section{Can the T'erms in $\mathbf{\Sigma} Y$ destroy euth other?}

18. It is important to prove that the order of magnitude of the constituents of $W(b, a)$ is not reduced by cancelling among the terms in $\mathbf{\Sigma Y}$.

We may write

$$
\phi(b, a)=\frac{1}{2} \Sigma(P a / P b)^{\ddagger}(P a)^{-\frac{1}{2}} \exp \int_{a}^{b} P^{\mathbf{k}} d x, \text { nearly, }
$$

where the summation now refers to the different paths $a b$ and also to the two initial values of $P^{3}$. Each of the paths may be deformed so as to pass through the zeros of $P$, and thus all the paths may be taken to coincide; each is divided into sections by the zeros of $P$ and the distinction between any two paths consists in a difference in the sign of $P^{\prime}$ on one or more of the sections.

The straight path for $x$ and corresponding path of $z=\int P^{\mathrm{i}} d x$ are exemplitied in a figure (Fig. 1). The lateral part of $P$ is supposed to be always of the sign $+\iota$
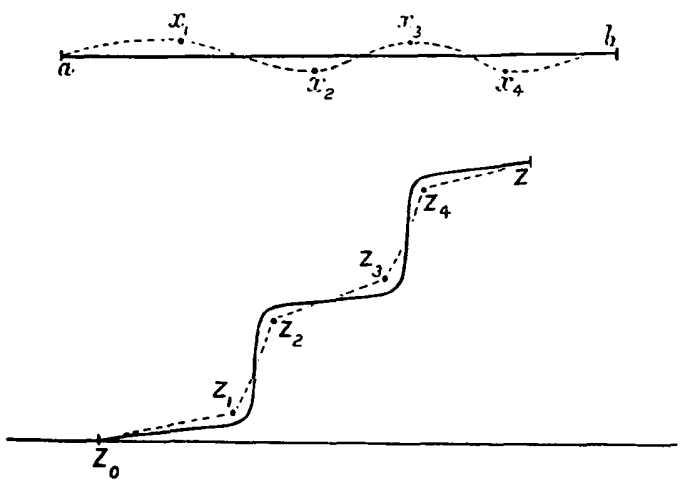

Fig. 1.

when $x$ is real, so that $x_{1}, x_{2}, x_{3}, x_{4}$, the zeros of $P$, lie on the two sides of the straight path alternately. The path of $z$ is zigzag with rounded corners, and $z_{0}, z_{1}, z_{2}, z_{3}, z_{4}, z$ correspond to $a, x_{1}, x_{2}, x_{3}, x_{4}, b$ respectively. The dotted line shows the deformed path for $x$, and its division into sections: in the $z$ plane the deformed path follows the dotied line if $x$ originally follows the straight path, so that the real part of $P^{\sharp}$ is of constant sign. If the $x$-path passes to the other side, say of $x_{1}$, then in the $z$-ptane the sections of the dotted path from $z_{1}$ onwards are to be reversed. The sections of the $z$-path are nearly horizontal and nearly vertical alternately. If we write $p$ for $\int P^{h} d x$ on a nearly horizontal section, and 
$q$ for $\int P^{\sharp} d x$ on a nearly vertical one, the real part in each being taken positive, then on the whole path

$$
\int_{a}^{b} P^{\mathbf{t}} d x=\Sigma( \pm p)+\Sigma( \pm q)
$$

and all possible combinations of the signs in this expression are to be taken into account in the summation in (12).

The number of sections is limited, being at most two more than the number of stationary values of $s / I$. Those terms in which one or more of the $p$ 's or $q$ 's are negative are comparatively small, and may be neglected, if the real part of $\int P^{\mathrm{d}} d x$ is great on each section.

Now we are supposing the real parts of $\lambda, \mu$ and therefore generally of $P$, to be $\| k^{2}$; let the lateral parts be $\| k k_{1}$, so that $k_{1} / k$ is small. Then the real and lateral parts of $P^{k}$ are $\| k, k_{1}$ on a $p$-section and $\| k_{1}, k$ on a $q$-section, and therefore we need to have $k_{1}$ great. If $k_{1}$ increases indefinitely with $k$, then the approximate value of $\phi(b, a)$ is

$$
\frac{1}{2}(P a P b)^{-4} \exp (\Sigma p+\Sigma q) \quad \text { or } \quad \frac{1}{2}(P a P b)^{-4} \exp \int_{a}^{b} \sqrt{ } P d x,
$$

as in the simpler case when the hyperbolic approximation is valid and the real part of $\int \sqrt{ } P d x$ is great; so that the terms of $\Sigma Y$ do not cancel.

This result holds if we are able to use the hyperbolic value for the Riccati function on each section of the path, but the proof fails when the path begins or ends so near a zero of $P$ that the argument of the Riccati function involved is finite or small. This cannot happen when $k_{1}$ is great in comparison with $k^{\mathrm{l}}$, and the error will not vitiate the proof if the argument of the Riccati function exceeds a certain tinite limit, that is, if $k_{1} k^{-3}$ exceeds a certain finite number: when this is not so, further investigation is needed.

\section{Another Method.}

19. The following method of combining the sections is slightly different, and may be used when the path $a b$ begins or ends near a zero of $P$. Let $x_{1}, x_{3}, \ldots, x_{n}$ be the zeros of $P$, occurring on the two sides of the real path $a b$ alternately, then

$$
W(b, a)=W\left(b, x_{n}\right) \ldots W\left(x_{2}, x_{1}\right) W\left(x_{1}, a\right) .
$$

Suppose to fix the ideas that $x_{1}$ is on the upper side of $a b$, then $x_{1} x_{8}$ is a $q$-section, $x_{2} x_{3}$ a $p$-section, and so on. We shall take the amplitude of $P$ to be nearly 0 on a $p$-section, and nearly $\pi$ on a $q$-section; the amplitudes of $-P_{1}^{\prime}, P_{2}^{\prime},-P_{3}^{\prime}, \ldots$ will be nearly 0 .

When $x$ is between $x_{1}$ and $x_{8}$, but not near either of them, we have the approximation

$$
1 \phi\left(x, \dot{x}_{9}\right)=\left(w^{\prime} w_{2}^{\prime}\right)^{-1} \omega(w, 0),
$$

where $\frac{2}{3} w^{\frac{2}{2}}=\int_{x_{a}}^{x} P^{i} d x, w^{\prime}=d w / d x$, and $w_{2}^{\prime}$ is the value of $w^{\prime}$ when $x=x_{2}$, namely $l_{2}^{\prime 4}$. 
Thus on the section $x_{1} x_{2}$ we have, by the approximation to $\omega$

$$
\phi\left(x, x_{a}\right)=A\left(w^{\prime} w_{?}^{\prime}\right)^{-\frac{1}{2}} w^{-\frac{1}{4}} \sinh \left(\frac{2}{3} w^{3}+a\right)=A P_{2}^{\prime-\frac{1}{4}} P^{-\frac{1}{4}} \sinh \left(a-\int_{x}^{x_{a}} P^{\prime} d x\right)
$$

and also

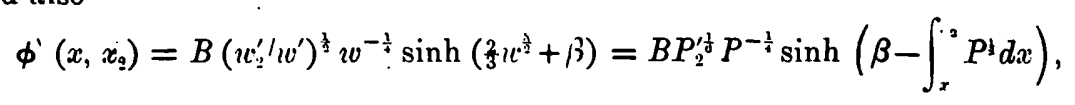

where $A, B, a, \beta$ have the meanings of $\S 10$. On the section $x_{:} x_{y}$,

$$
\begin{aligned}
& \phi\left(x, x_{9}\right)=A_{1}\left(u^{\prime} u_{2}^{\prime}\right)^{-\frac{1}{4}} w^{-\frac{1}{2}} \sinh \left(\frac{\frac{2}{3}}{3} w^{\frac{2}{2}}-a_{1}\right)=A_{1} P_{2}^{\prime-\frac{1}{3}} P^{-\frac{1}{4}} \sinh \left(\int_{x_{1}}^{r} P^{1} d x-a_{1}\right), \\
& \phi^{\prime}\left(x, x_{3}\right)=B_{1} P_{2}^{\prime \frac{1}{2}} P^{-\frac{1}{2}} \sinh \left(\int_{x_{2}}^{x} P^{\mathrm{s}} d x-\beta_{1}\right),
\end{aligned}
$$

where, since the distinction between $\sinh$ and cosh disappears, we may put

$$
a_{1}=\beta_{1}=0, \quad A_{1}=A e^{j_{1 \times}}, \quad B_{1}=-B e^{i k \pi} ;
$$

thus $A_{1}, B_{1}$ are real. These approximate equations may be differentiated with respect to $x$; there are similar expressions in the domains of $x_{4}, x_{\theta}, \ldots$.

When $x$ is between $x_{1}$ and $x_{4}$, we use an auxiliary variable $w$ which decreases as $x$ increases, and we have

$$
\phi\left(x, x_{s}\right)=\left(w^{\prime} w_{3}^{\prime}\right)^{-\frac{1}{1}} \omega(w, 0),
$$

where now $\frac{2}{3} w^{\frac{3}{3}}=\int_{x}^{x_{2}} P^{\mathrm{j}} d x, w^{\prime}=d w / d x$, and $w_{3}^{\prime}$ is the value of $w^{\prime}$ when $x=x_{3}$, that is, $-\left(-P_{3}^{\prime}\right)^{4}$.

Hence on the section $x_{2}, x_{3}$, approximately,

$$
\begin{aligned}
& \phi\left(x, x_{3}\right)=-A_{1}\left(-P_{3}^{\prime}\right)^{-\frac{1}{3}} P^{-\frac{1}{4}} \sinh \left(\int_{x}^{x_{3}} P^{4} d x-a_{1}\right), \\
& \phi^{\prime}\left(x, x_{3}\right)=B_{1}\left(-P_{3}^{\prime}\right)^{\frac{1}{6}} P^{-\frac{1}{4}} \sinh \left(\int_{x}^{x_{3}} P^{\frac{1}{4}} d x-\beta_{1}\right),
\end{aligned}
$$

and on the section $x_{3} x_{4}$,

$$
\begin{aligned}
& \phi\left(x, x_{3}\right)=-A\left(-P_{3}^{\prime}\right)^{-\frac{1}{d}} P^{-\frac{1}{4}} \sinh \left(a-\int_{x_{3}}^{x} P^{\mathbf{t}} d x\right), \\
& \phi^{\prime}\left(x, x_{3}\right)=B\left(-P_{3}^{\prime}\right)^{\frac{1}{4}} P^{-\frac{1}{d}} \sinh \left(\beta-\int_{x_{3}}^{x} P^{\mathbf{l}} d x\right),
\end{aligned}
$$

with similar expressions for the domains of $x_{1}, x_{5}, \ldots$.

Now $W\left(x_{2}, x_{1}\right)=W\left(x_{2}, x\right) W\left(x, x_{1}\right)$, where $x$ is on $x_{1} x_{9}$ :

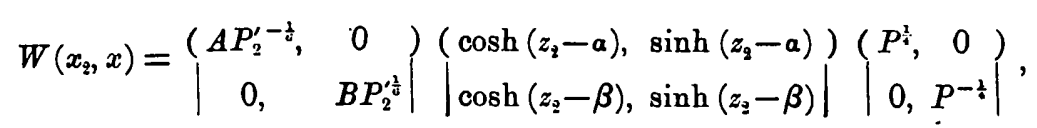

- There is a difficulty in getting the signs right in these expressions; it may be overcome by taking a prrticular form for $P$, say $-m^{3}\left(x-x_{3}\right)$ where $m$ is positive. 
1906.] HaRMonic expansions of Functions of two variables.

where

$$
z_{2}=\int_{x}^{x_{2}} P^{i} d x
$$

and $W\left(x, x_{1}\right)=\left\{\begin{array}{cc}P^{-\frac{1}{4}}, & 0 \\ 0, & P^{\frac{1}{4}}\end{array}\right\}\left\{\begin{array}{l}\sinh \left(z_{1}-\beta\right), \sinh \left(z_{1}-a\right) \\ \cosh \left(z_{1}-\beta\right), \cosh \left(z_{1}-a\right)\end{array}\right\}\left(\begin{array}{cc}B\left(-P_{1}^{\prime}\right)^{\frac{1}{2}}, & 0 \\ 0, & A\left(-P_{1}^{\prime}\right)^{-\frac{1}{\alpha}}\end{array}\right)$,

where

$$
z_{1}=\int_{r,}^{x} P^{\mathrm{I}} d x
$$

thus

$$
\begin{aligned}
W\left(x_{2}, x_{1}\right)=\left\{\begin{array}{cc}
A P_{2}^{\prime-\frac{2}{4}}, & 0 \\
0, & B P_{2}^{\prime \frac{3}{3}}
\end{array}\right)\left\{\begin{array}{ll}
\sinh \left(z_{1}+z_{2}-\alpha-\beta\right), & \sinh \left(z_{1}+z_{2}-2 \alpha\right) \\
\sinh \left(z_{1}+z_{2}-2 \beta\right), & \sinh \left(z_{1}+z_{2}-\alpha-\beta\right)
\end{array}\right) \\
\times\left(\begin{array}{cc}
B\left(-P_{1}^{\prime}\right)^{\frac{1}{2}}, & 0 \\
0, & A\left(-P_{1}^{\prime}\right)^{-\frac{1}{b}}
\end{array}\right),
\end{aligned}
$$

where $\quad z_{1}+z_{2}=\int_{x_{1}}^{x_{2}} P^{1} d x$.

This is the typical expression for a $q$-section; if $x_{1} x_{3}$ had been a $p$-section we should have had $P_{1}^{\prime}$ for $-P_{1}^{\prime}$ and $-P_{2}^{\prime}$ for $P_{2}^{\prime}$, and also $A_{1},-B_{1}, a_{1}, \beta_{1}$ for $A, B$, $\boldsymbol{a}, \boldsymbol{\beta}$.

Since $x_{2} x_{8}$ is a $p$-section, the product $W\left(x_{3}, x_{2}\right) W\left(x_{2}, x_{1}\right)$ is now resolved into six factors of which the two middle ones are

$$
\left(\begin{array}{cc}
-B_{1} P_{2}^{\prime \frac{1}{4}}, & 0 \\
0, & A_{1} P_{2}^{\prime-\frac{1}{d}}
\end{array}\right)\left(\begin{array}{cc}
A P_{2}^{\prime-\frac{\mathrm{d}}{d}}, & 0 \\
0, & B P_{2}^{\prime \frac{d}{d}}
\end{array}\right),
$$

whose combined effect is simply a multiplication by $A_{1} B$, since $A B_{1}=-A_{1} B$. All such factors may therefore be put outside the product.

Moreover in a $p$-section, such as $x_{z} x_{3}$, the middle factor is simply

$$
(1,1) \text {, }
$$

multiplied by the common factor $\sinh \int_{x_{*}}^{x_{2}} P^{4} d x$, which may also be put outside. Thus now, apart from factors at the two ends, every alternate matrix in the sequence is of the form

$$
(1,1)
$$

But

$$
\left(\begin{array}{l}
1,1 \\
1,1
\end{array}\right)\left(\begin{array}{ll}
a, b \\
c, d
\end{array} \mid\left(\begin{array}{l}
1,1 \\
1,1
\end{array} \mid=(a+b+c+d)\left(\begin{array}{l}
1,1 \\
\mid 1,1
\end{array} \mid,\right.\right.\right.
$$

and thus if a $q$-section lies between two $p$-sections we may strike out two matrices from the sequence, putting outside the factor

$$
\begin{aligned}
2 \sinh (q-a-\beta) & +\sinh (q-2 \alpha)+\sinh (q-2 \beta) \\
& =4 \sinh (q-\alpha-\beta) \cosh ^{2} \frac{1}{2}(\alpha-\beta)=4 \iota \cosh q /\left(-A^{2} B^{2}\right) \\
& =4 \cosh q / A_{1}^{2} B^{2} .
\end{aligned}
$$


This is consistent with the former result, if we take account of the factors $A_{1} B$ and $\sinh p$ that have been put outside already, and substitute $\exp q, \exp p$ for $2 \cosh q, 2 \sinh p$.

\section{Case of Terminal Short Sections.}

20. The factors corresponding to the terminal sections are also found on examination to agree with those already found, when those sections are of finite length, so that the approximation to $\omega$ is available; this approximation cannot, however, be used for a section so short that the argument of the corresponding $\omega$ does not become infinite with $k$. Denoting a short section by $s$, we have to consider the following possible arrangements :-

$s p, p s, s q, q s, s p s, s q s, s p q, p q s, s q p, q p s, s p q s, s q p s, s q p q, q p q s, s q p q s$;

here $s p q$, for instance, means a short section $\omega_{1} x_{1}$ followed by a $p$-section $x_{1} x_{2}$, and then by a $q$-section $x_{i} b$ : we have shewn that the sequence $p q p$ can be replaced by $p$ and an outside factor, so that any possible sequence can be reduced to one of those written, so far as concerns the matrices involved. The matrix corresponding to $p$ is $(1,1)$ with an outside factor. There is no reduction in the 1,1 !

order of magnitude on account of these outside factors, as has been pointed out.

When a $p$-section is between two others we have a product of the form

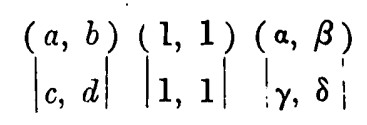

or

$$
\left\{\begin{array}{ll}
(a+b)(a+\gamma), & (a+b)(\beta+\delta)) \\
(c+l)(a+\gamma), & (c+d)(\beta+\delta)
\end{array} \mid .\right.
$$

Here the orders of magnitude are found at once if we know those of $a+b, c+d$, $a+\gamma, \beta+\delta$ which are the coefficients in the separate products

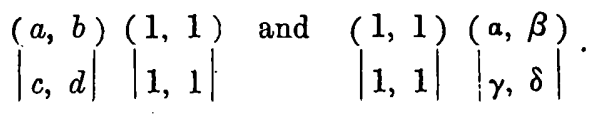

Hence the consideration of the different arrangements is simplified, for they all depend on the following :-

$$
s q s, s p, s q, s q p
$$

and the last three reversed, $p s, q s, p q s$.

For an arrangement ending in $q s$ (or, reversed, beginning with $s q$ ) we may make use of the formulæ of $\S \S 3,4$, for instance,

$$
\boldsymbol{\phi}(b, a) \boldsymbol{\phi}_{1}^{\prime}(b, a)-\boldsymbol{\phi}_{1}(b, a) \boldsymbol{\phi}^{\prime}(b, a)=\int_{a}^{b}\left(P_{1}-P\right) \boldsymbol{\phi}(x ; a) \phi_{1}(x, a) d x
$$

where the suffix 1 now indicates the change of $\lambda, \mu$ into the conjugate quantities. 
Here $P_{1}-P$ is of constant sign and always $\| k k_{1}$. Also $\phi(x, a) \phi_{1}(x, a)$ is always positive but may oscillate about a mean value \|

$$
K^{\prime \prime}\left\{P(x) P_{1}(x)+k^{3}\right\}^{-\frac{1}{4}} \exp \int_{a}^{x}\left(\sqrt{ } P+\sqrt{ } P_{1}\right) d x
$$

where $K$ does not depend on $x$, and the term $k_{*}^{*}$ in the bracket is added to provide for the case when $P$ falls below the order of $k^{4}$.

There is a zero of $P$ near to $b$, suppose at $x_{n}$. The period of oscillation of $\omega(x, 0)$ is variable but finite so long as $: t$ is finite, and hence in the neighbourhood of $x_{n}$ the period of oscillation of $\psi$ is greater than in the body of the $q$-segment and reaches the order of magnitude of $k^{-1}$, which in the present case is not higher than $1 / h_{1}^{2}$.

In the neighbourhood of $x_{n}, P \| k^{*}\left|x-x_{n}\right|$, and the lateral part, of $P \| k k_{1}$ on the path, so that on the path the real part of $\sqrt{ } P$ is $\| k \sqrt{ }\left|x-x_{n}\right|$ on the short $p$-section and $\| k_{1} / \sqrt{ }\left|x-x_{n}\right|$ on the $q$-section; the integral of $\sqrt{ } P+\sqrt{ } P_{1}$ is therefore finite on the $p$-section so long as $k\left(x-x_{n}\right)^{\frac{3}{2}}$ is finite, that is, $x-x_{n}$ $\| k^{-j}$, and on the $q$-section so long as $k_{1}\left(x-x_{n}\right)^{t}$ is finite, that is, $x-x_{n} \| k_{1}^{-2}$.

Hence in $\int_{a}^{b}\left(P_{1}-P\right) \phi(x, a) \phi_{1}(x, a) d x$, the argument of the exponential factor in $\phi \phi_{1}$ is only reduced from its maximum by a finite quantity if $x$ travels back from the upper limit $b$ through a distance of the order of $k_{1}^{-2}$, that is, the order of magnitude of the exponential factor is at its maximum through such an interval; such an interval can also be made to inclnde complete periods of oscillation, so that we may neglect the oscillation* in estimating the order of magnitude of the integral: we shall also neglect the whole path $a b$ except this interval at the upper limit. $\dagger$

The integral is therefore at least $\|$

$$
k k_{1} K^{2} \exp \int_{a}^{\prime \prime}\left(\sqrt{ } P+\sqrt{ } P_{1}\right) d x \int\left\{P(x) P_{1}(x)+k^{3}\right\}^{-4} d x
$$

the integration in the last factor being taken over the interval at the upper limit. The last factor is then $\|$

$$
\int\left\{k^{4}\left|x-x_{n}\right|^{2}+k^{8}\right\}^{-\frac{1}{4}} d x \text { or } \quad k^{-1} \int\left\{\left|x-x_{n}\right|^{2}+k^{-\frac{3}{3}}\right\}^{-\frac{1}{2}} d x
$$

* On the same principle, although sinz $k x$ is a violently oscillating function, $\int_{a}^{\beta} \sin ^{2} k x d x$ is $\| \beta-\alpha$, that is, $\|$ the range multiplied $b y$ the mean value, unless the range is a small fraction of the period of oscillation and is near to a zero of the subject of integration.

+ Similarly in estimating the mere order of magnitude of $\int_{0}^{1} e^{k x} d x$ one may take the upper purt $\int_{1-k^{-1}}^{1} \epsilon^{k x} d x$, and neglect the rest. The subject is now always $>e^{k} / e$, and therefore $\| e^{k}$, while the range is $1 / k$. Thus the integral is at least $\| e^{k} / k$. 
which comes out $i\left(\cdots_{1}\right)^{-1}$. The result is therefore that

$$
\phi(b, a) \phi_{1}^{\prime}(b, a)-\phi_{1}(b, a) \phi^{\prime}(b, a)
$$

is at least \|

$$
K^{2} \exp \int_{a}^{b}\left(\sqrt{ } P+\sqrt{ } P_{1}\right) d x
$$

and, since $\phi \phi_{1}^{\prime}$ and $\phi_{1} \phi^{\prime}$ are conjugate imaginaries, each of them must be of this order.

But $\phi, \phi_{1}$ are at most $\| K k^{-j} \exp \int_{a}^{b} \sqrt{ } P d x$

and

$$
\phi^{\prime}, \phi_{1}^{\prime} \text { are at most } \| K k^{\dagger} \exp \int_{a}^{b} \sqrt{ } P d x \text {. }
$$

. Hence we must have

$$
\begin{aligned}
& \phi, \phi_{1} \| K k^{-3} \exp \int_{a}^{b} \sqrt{ } P d x, \\
& \phi^{\prime}, \phi_{1}^{\prime} \| K k^{3} \exp \int_{a}^{b} \sqrt{ } P d x
\end{aligned}
$$

that is, the oscillation does not affect the order of magnitude, when the arrangement begins with $s q$ or ends with $q s$.

21. An arrangement ending with $p s$ may be treated as follows. The $p$-segment gives a factor $(1,1)$ to whose left are the matrices

$$
|1,1|
$$

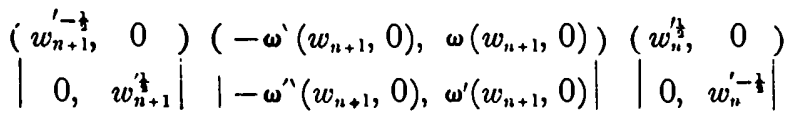

$$
\begin{aligned}
& \times\left(\begin{array}{cc}
A_{1}\left(-P_{n}^{\prime}\right)^{-\frac{2}{2}}, & 0 \\
0, & -B_{1}\left(-P_{n}^{\prime}\right)^{\frac{1}{11}}
\end{array}\right),
\end{aligned}
$$

where $\quad \frac{2}{3} w_{n+1}^{\frac{3}{3}}=\int_{b}^{y_{n}} p^{\ddagger} d x$ and $w_{n}^{\prime}=-\left(-P_{n}^{\prime}\right)^{k}$.

The product of these five matrices is

$$
\iota\left(\begin{array}{cc}
w_{n+1}^{\prime-1}, & 0 \\
0, & w_{n+1}^{\prime}
\end{array} \mid\left\{\begin{array}{ll}
-A_{1} \omega^{\prime}+B_{1} \omega, & -A_{1} \omega^{\prime}+B_{1} \omega \\
-A_{1} \omega^{\prime \prime}+B_{1} \omega^{\prime}, & -A_{1} \omega^{\prime \prime}+B_{1} \omega^{\prime}
\end{array} \mid,\right.\right.
$$

where the constituents in the right-hand factor are constant multiples of $\chi\left(w_{n+1}\right)$ and $\chi^{\prime}\left(w_{n+1}\right)(\S 13)$. Now in the present case $w_{i+1}$ is small or finite and thus $\chi, \chi^{\prime}$ are not great, nor will either be small except when $w_{n+1}$ is near one of its zeros: it has been proved that these zeros are real, whereas $w_{n+1}$ will be found 
to have a lateral part at least $\| k_{1} k^{-k}$. Hence the order of $\chi\left(v_{n+1}\right)$ or $\chi^{\prime}\left(v_{n+1}\right)$ is at least $k_{1} k^{-\frac{1}{2}} .^{*}$

Thus in the case of a short terminal section there is no reduction in order of magnitude when the next section is of the $q$-kind, but there may be a reduction by a factor $\| k_{1} k^{-k}$ when the next section is of the $p$-kind; this may, in fact, happen at both ends of the path simultaneously.

The short section may be either forwards or backwards, so that the discussion serves even when $x_{n}$ is near the terminus $a$ or $b$ but on the far side.

\section{Stationary Values of $S / T$. Riccati case.}

22. When $S / T$ has a stationary value, say $p$, there appear to be further complications when $\mu / \lambda$ is nearly equal to $p$, but, in fact, the foregoing approximations give all that is wanted for our purpose.

Let $c$ be the value of $x$ for which $S-p T=0$, and $m$ the order of multiplicity of the factor $x-c$. Let $\lambda=\xi+\imath \eta, \mu=\xi+\iota \omega$, then

$$
P=\alpha(x-c)^{m}+\beta+\iota \gamma \text {, }
$$

where

$$
\begin{aligned}
a(x-c)^{m} & =\xi(-S+p T), \\
\beta & =(-p \xi+\zeta) T-X, \\
\gamma & =-\eta S+\omega T .
\end{aligned}
$$

Thus when $|x-c|$ is below a certain finite value, $\alpha$ is of constant sign and of the order of $k^{2}, \gamma$ is of constant sign and of the order of $k k_{1}, \beta$ is either finite, and therefore of lower order than $\gamma$, or else is of constant sign, namely that of $-p \xi+\zeta$ : suppose $k_{\mathrm{q}}$ then to be the order of magnitude of $\beta+\imath \gamma$.

$P$ vanishes at a cluster of $n$ points which are, approximately, placed symmetrically round a circle with centre $c$; the path of integration passes diametrically across the circle; let $x_{1}$ be the nearest of the $m$ points where $P$ vanishes to one end of this diameter, and take the Riccati approximation with origin at $x_{1}$, that is, put

$$
\frac{2}{3} w^{\frac{3}{2}}=\int_{x_{1}}^{x} \sqrt{ } P d x
$$

The relative magnitude of the error is $(\$ \S 8,15)$ that of

$$
\int\left|\{w, x\} d x / w^{\prime} \sqrt{ } w\right| \text {. }
$$

- The method which was applied to the other kind of short section would also give this result, the only change being that the interval at the upper limit of which account is taken in

$$
\int_{a}^{b}\left(P_{1}-P\right) \phi(x, a) \phi_{1}(x, a) d x
$$

is now $\| k^{-1}$ instead of $\| k_{1}^{-2}$, so that the integral is now $\|$

$$
k_{1} k-k K^{2} \exp \int_{a}^{b}\left(\sqrt{ } P+\sqrt{ } P_{1}\right) d x .
$$

Thus the lowering of order is possible; the method of the text has the advantage of shewing when it happens. 
The distances between the $m$ points of the cluster and their distances from $c$ are $\|\left(k_{2} / k^{2}\right)^{1 / m}$. Suppose $x-x_{1}$ not to be above this order, and the distance of $x$ from $c$ or any other point of the cluster not to be below it. Then $P^{\prime}$ is $\| \mathrm{a}(x-c)^{m-1}$, that is $\| k_{1}\left(k^{4} / k_{8}\right)^{1 / m}$;

$$
\begin{aligned}
& P \| k_{9}\left(k^{2} / k_{g}\right)^{1 / m}\left(x-x_{1}\right), \\
& w \| k_{9}^{4}\left(k^{3} / k_{g}\right)^{1,3 m}\left(x-x_{1}\right), \\
& w^{\prime} \| k_{2}^{3}\left(k^{2} / k_{g}\right)^{1,3 m,}
\end{aligned}
$$

since $\frac{2}{3} w^{\frac{3}{3}}=\int_{x_{2}}^{x} \sqrt{ } P d x$ and $w^{3} w=P:$ also since $w$, as a function of $x$, has no singularity in a domain whose linear dimensions $\|\left(k_{2} / k^{2}\right)^{1 / n}$ and is of the order just given, the orders of $w^{\prime \prime}, w^{\prime \prime \prime}$ are at most $k_{9}^{\dagger}\left(k^{z} / k_{9}\right)^{4 / 3 m}, k_{3}^{\dagger}\left(k^{2} / k_{8}\right)^{7 / 3 m}$.

Thus $w^{\prime \prime \prime} / w^{\prime}$ and $\left(w^{\prime \prime} / w^{\prime}\right)^{2}$, and therefore $\{w, x\}$, are not of higher order than $\left(k^{2} / k_{2}\right)^{2 ! m}$.

The other factor under the integral sign is

$$
d x / w^{\prime} \sqrt{ } w=d x / \sqrt{ } P=\frac{2}{P^{\prime}} d \sqrt{ } P,
$$

and thus the integral on the part of the path now in question is \|

$$
\left(k^{8} / k_{2}\right)^{2 / m} \cdot k_{2}^{-1}\left(k^{2} / k_{2}\right)^{-1 / m} \cdot k_{2}^{\mathrm{b}},
$$

the first factor being given by $\{w, x\}$, the second by $2 / P^{\prime}$, and the third by the length of path of the point $\sqrt{ } P$. The product is $k^{2 m} k_{2}^{-3-1 ; m}$, and this is therefore the relative order of error when all points of the path are at distances not below the order of $\left(k_{2} / k^{2}\right)^{\prime \prime m}$ from $c$ and from all the points of the cluster but $x_{1}$, and not above this order from $x_{1}$.

\section{Hyperbolic Case.}

23. To combine the section thus limited with other parts of the given path we can use the hyperbolic approximation, the error in which ( $(7)$ is comparatively small on any section, if $\int\left|P^{-\frac{1}{2}} \frac{d^{z}}{d x^{2}} P^{-\frac{1}{3}} d x\right|$ is small on that section. This integral does not exceed the product of the greatest value of $\left|P^{-1}\right|$ and the length of path of the point $d / d x P^{-t}$ or of $P^{\prime} / 4 P^{\frac{z}{4}}$.

Now, when the real part of $P$ vanishes, $P \| k k_{1}$, which may or may not be lower than $k_{2}$.

If $k k_{1} \| k_{2}$, or if the section does not extend to any point where $P$ is below the order of $k_{\mathfrak{g}}$, the greatest value of $\left|P^{-1}\right|$ is $\| k_{2}^{-\frac{1}{4}}$, and the length of path of $P^{\prime} / 4 P^{t}$ is $\|$ the greatest value of $\left|P^{\prime} / P^{\mathbf{q}}\right|$, that is, $\| k_{2}^{-\frac{1}{3}}\left(k^{9} / k_{3}\right)^{1 / m}$. Hence under these restrictions the relative magnitude of error for the section is $k_{2}^{-i-1 / m} k^{2 / m}$.

If the section is not thus restricted, but includes the point where the real part of $P$ vanishes, the greatest value of $\left|P^{-1}\right|$. is $\|\left(k k_{1}\right)^{-t}$ and the length of path is $\|\left(k k_{1}\right)^{-\frac{4}{4}} k_{8}\left(k^{2} / k_{2}\right)^{1 / m}$, so that the relative order of possible error is raised to $\left(k k_{1}\right)^{-\frac{i}{3}} k_{2}^{1-1 / m} k^{2 / m}$. 
Even this tends to zero as $k$ increases, if $k_{2} \leqslant k k_{1}^{2}$, and if $k_{1}$ increases indefinitely with $k$, for it may be written $\left(k_{2} / k k_{1}^{2}\right)^{1-1 / m}\left(k / k_{1}\right)^{-1+1 ; m} k_{1}^{-1 / m}$ and $m=2$, at least.

\section{Combination of the Two Cases.}

24. These results are to be applied as follows, when $\zeta / \xi$ is nearly equal to $p$. Let $\zeta-p \xi=k_{s}$, and $\eta=k k_{1}$, where $k_{1}$ increases indefinitely with $k$; the value of $\omega$ is such that $-\eta S+\omega T$ is of constant sign, and always $\| k k_{1}$.

If $k_{\mathrm{s}} \leqslant k k_{1}^{2}$, the hyperbolic approximation is valid. If $k_{3}>k k_{1}^{2}$, so that $k_{2}=k_{3}$, let $r=\left|x_{1}-c\right|$, the radius of the circle on which the $m$ zeros of $P$ approximately lie. Divide the path into sections at the points $c \pm \frac{3}{2} r, c \pm \frac{1}{2} r$. Between $c \pm \frac{1}{2} r$, and outside $c \pm \frac{3}{2} r$, the hyperbolic approximation is taken, since $P$ does not fall below the order of $k_{2}$. Between $c-\frac{3}{2} r$ and $c-\frac{1}{2} r$ or $c+\frac{1}{3} r$ and $c+\frac{3}{2} r$ a Riccati approximation may be used, with origin at the nearest zero of $P$ in either case. The matrices corresponding to the different sections are then to be compounded, and the error in the result is relatively $\|$

$$
k^{2 / m} k^{-1-1 / m} \text { or }\left(k_{3} / k\right)^{-1-1 / m} k^{-1+1 / m}
$$

which tends to zero as $k$ increases, since $k_{s} / k$ increases indefinitely with $k$ and $m \nless 2$.

For the Riccati functions we may substitute their approximate values in terms of hyperbolic fulctions, since the arguments are great, as will appear from the following discussion of the present nature of the sections into which the path was divided in $\S 18$. Then the resulting approximate formulæ will be the same as in the simpler case, being formed in exactly the same way.

If $m$ is even and the circumference of a circle is divided into $m$ equal parts the points of division are diametrically opposite in pairs. Thus the origins in the two Riccati approximations are almost diametrically opposite, and the diameter between them forms a section which may be of either kind, $p$ or $q$, but is short. It has to be sliewn that the real part of $\int \sqrt{ } P d x$ on this section is great.

If $m$ is odd the points of division of the circle are not diametrically opposite. If at one end of the diameter the Riccati approximation must be used on account of its origin coming so near the path that $P$ falls below the order of $k_{9}$ on the path, then at the other end of the diameter this does not happen, and the hyperbolic approximation will serve: hence there is no short section when $m$ is odd.

There will be a terminal short section for any value of $m$ when $c$ falls at one of the termini of the path, but as $c$ does not depend on $k$ it cannot tend to one of the termini as a limit, and some complication is thus saved.

\section{Short Sections.}

25. When $x=c, P=\beta+\iota \gamma$, and there are two cases according as $\beta$ is great and positive or great and negative. When $\beta$ is finite, or indeed below BER. :2. VOL. 5. No. 972 . 
the order of $k k_{1}^{2}$, there is no short section, for we use the hyperbolic approximation.

In the other two cases we need to discuss the order of magnitude of the real part of $\int_{c}^{x_{1}} \sqrt{ } P d x$.

Now, so long as $x-c \|\left(k_{2} / k^{2}\right)^{1 / m}$ or less, we have $P^{\prime \prime}$ at most $\left\|a(x-c)^{m-2}\right\|$ $k^{2}\left(k_{2} / k^{2}\right)^{1-2 / 1 n}$. Thus, by two integrations, since $P=0$ at $x_{1}$,

$$
P-P_{1}^{\prime}\left(x-x_{1}\right) \| k^{2}\left(k_{2} / k^{2}\right)^{1-2 ; m}\left(x-x_{1}\right)^{2},
$$

where $P_{1}^{\prime}$ is the value of $P^{\prime}$ at $x_{1}$.

Hence, since $P_{1}^{\prime} \| k^{2}\left(k_{2} / k^{2}\right)^{1-1^{\prime} m}$, we have

and

$$
\begin{gathered}
\sqrt{ } P-\sqrt{ } P_{1}^{\prime}\left(x-x_{1}\right)^{\prime} \| k\left(k_{2} / k^{2}\right)^{\frac{1}{-3 / 2 m}\left(x-x_{1}\right)^{\frac{4}{2}},} \\
\int_{x_{1}}^{x} \sqrt{ } P d x-\frac{2}{3} \sqrt{ } P_{1}^{\prime}\left(x-x_{1}\right)^{\frac{3}{2}} \| k\left(k_{2} / k^{2}\right)^{j-3 / 2 m}\left(x-x_{1}\right)^{\frac{3}{2}} .
\end{gathered}
$$

Now, if we take a triangle $x_{1} e f$, having its base ef on the real axis, its vertical angle equal to $\frac{2}{3} \pi$ and neither of its base angles less, say, than $\frac{1}{12} \pi$, the amplitudes of $\frac{a}{3} \sqrt{ } P_{1}^{\prime}\left(x-x_{1}\right)^{\frac{1}{4}}$ at $e, f$ differ by $\pi$, and cannot in all positions of $e, f$ be $\pm \frac{1}{2} \pi$ : thus we may choose positions of $e, f$ where $\frac{2}{3} \sqrt{ } P_{1}^{\prime}\left(x-x_{1}\right)^{3}$ has substantial real parts with opposite signs. Since at $e, f, x-x_{1} \|$ the lateral part of $x_{1}-c$, that is $\| k k_{1} / k_{2}\left(k_{2} / k^{2}\right)^{1 / n}$, the absolute value and real part of $\frac{9}{3} \sqrt{ } P_{1}^{\prime}\left(x-x_{1}\right)^{\frac{1}{2}}$ are $\| k\left(k_{2} / k^{3}\right)^{1-1 / 2 m}\left(k k_{1} / k_{9}\right)^{\frac{3}{3}}\left(k_{2} / k^{2}\right)^{3 / 2 m}$, that is, $k\left(k_{2} / k^{9}\right)^{1+1 / m}\left(k k_{1} / k_{9}\right)^{\frac{1}{4}}$, while $\int_{x_{1}}^{x} \sqrt{ } P d x$ differs from $\frac{2}{3} \sqrt{ } P_{1}^{\prime}\left(x-x_{1}\right)^{\frac{1}{2}}$ by a quantity $\| k\left(k_{2} / k^{2}\right)^{1+1 / m}\left(k k_{1} / k_{8}\right)^{2}$, which may be neglected since $k_{2} / k k_{1}$ increases indefinitely with $k$. Thus the real part of $\int_{x_{1}}^{x} \sqrt{ } P d x$ has opposite signs at $e, f$ and must vanish at some intermediate point, say $g$. Also the distances ce, $c f$ are equal to $r$ in the limit. Thus we have real part of

$$
\int_{0+1 r}^{x_{1}} \sqrt{ } P d x=\text { R.P. of } \int_{c+i r}^{0} \sqrt{ } P d x>\text { R.P. of } \int_{c+l, r}^{c+q r} \sqrt{ } P d x
$$

In this integral the range $\|\left(k_{2} / k^{g}\right)^{1 / \text { in }}$ and the real part of the subject is $\| \sqrt{ } k_{q}$ if $\beta$ is positive and $\| k k_{1} / \sqrt{ } k_{9}$ if $\beta$ is negative. The real part of the integral is therefore $\| k_{g}^{i+1 / m} k^{-2 / m}$ and $k_{1} k_{9}^{-1+1 / m} k^{1-2 ; m}$ in the two cases respectively: in each case it increases indefinitely when $k_{1}, k_{3} / k$, and $k^{2} / k_{2}$ do so.

The limits in the integral have been taken to be $c+\frac{1}{2} r$ and $x_{1}$; the same method would have applied if they had been $x_{1}$ and $c+\frac{3}{2} r$, or $c$ and $x_{1}$, or if we had taken the other Riccati origin instead of $x_{1}$.

Thus the arguments in the Riccati approximations must be great, since the corresponding values of $\int \sqrt{ } P d x$ are great: we are therefore entitled to use the hyperbolic approximate values of the Riccati functions. 
Also, if we go back to the form $(\S 18)$,

$$
\frac{1}{2} \Sigma \exp \{\mathbf{\Sigma}( \pm p)+\mathbf{\Sigma}( \pm q)\}(P a P b)^{-t},
$$

the real part in each section, $p$ or $q$, increases indefinitely with $k$ in all cases that come within the scope of the last investigation $(\$ \S 24,2.5)$. Thus the whole expression is $\|$ its greatest term

$$
\frac{1}{2}(P a P b)^{-1} \exp \int_{a}^{b} \sqrt{ } P d x
$$

in which all the upper sigus are taken, that is, where the integration is along the straight path, the real axis.

\section{Approximate Values of $\Theta$ and $\Phi$.}

26. Using the hyperbolic formulæ of $\S 7$, we have as the approximate value of $\theta$,

$$
\begin{aligned}
{\left[E_{1}\{P(0) P(1)\}^{-\frac{1}{4}}\right.} & \left.-E_{4}\{P(0) P(1)\}^{\frac{1}{4}}\right] \sinh \int_{0}^{1} P^{\frac{1}{3}} d x \\
& +\left[E_{2}\{P(1) / P(0)\}^{\frac{1}{4}}-E_{3}\{P(0) / P(1)\}^{\frac{1}{2}}\right] \cosh \int_{0}^{1} P^{\frac{1}{2}} d x-2 E .
\end{aligned}
$$

Suppose $E_{4} \neq 0$. Then the predominant term in $\theta$ is the one that contains $E_{4}$, the other terms merge in the error, and thus the approximation to $\theta$ is $-E_{4}\{P(0) P(1)\}^{\ddagger} \sinh \int_{0}^{1} P^{\frac{1}{2}} d x$ under the restrictions stated in $\$ 7$ as necessary for the validity of the hyperbolic approximation.

If the real part of $P$, and consequently that of $P^{t}$, is positive and great along the whole $x$-path, the hyperbolic sine and cosine of $\int_{0}^{1} P^{\frac{1}{3}} d x$ are great also, and thus $\theta$ cannot vanish.

Also, if $\lambda, \mu$ are such that the lateral part of $\int_{0}^{1} P^{3} d x=$ an odd multiple of $\frac{1}{2} \iota \pi$, the absolute value of the hyperbolic sine is not less than 1 , and $\theta$ cannot vanish for values of $\lambda, \mu$ in the neighbourhood.

Thus the formulæ of $\$ 7$ enable us to say that in two important cases $\theta$ cannot vanish. In conjunction with the results of $\$ \$ 8-25$, they also enable us to form an estimate of the orders of magnitude of the factors $\Phi(x, t) / \Theta$ and $\Psi(y, u) / Z$ of the subject of integration in $\$ 6$.

The approximate values found in $\$ 15$ by means of the Riccati functions were substantially the same as those of $\$ 7$, unless $P / k^{2}$ was small at an end of the path; it was assumed that the values of $\lambda, \mu$ were such that the lateral part of $P$ was $\| k k_{1}$ and of constant sign, $k_{1}$ increasing indefinitely with $k$. 
If $P / k^{2}$ is small, say when $x=0$, the same expression gives at least the true order of magnitude, provided when $P / k^{\mathbf{s}}$ is small we substitute $k^{ \pm \frac{1}{3}}$ for $P(0)^{ \pm \mathfrak{t}}$ in the formulæ, except that $(\S 21)$ when the next section is of the $p$-kind there may be a loss in order of magnitude by a factor $\| k_{1} k^{-t}$, either in the pair $\phi(1,0), \phi^{\prime}(1,0)$ or the pair $\phi^{\prime}(1,0), \phi^{\prime \prime}(1,0)$. These changes do not affect the predominance of the term $E_{4} \phi^{\prime \prime}(1,0)$.

In $\Phi(x, t)$ the predominant term may be $E_{4} \phi^{\prime}(1, t) \phi^{\prime}(x, 0)$ or $E \phi(t, x)$ according to the positions of $x, t$ on the path 0,1 . The latter would, for instance, be the most important if we had $x=0, t=1, P$ being great and positive. When the $E_{4}$ term predominates in $\Phi$, we have

$$
\Phi(x, t)=-E_{4}\{P(0) P(1) / P(t) P(x)\}^{\frac{1}{t}} \cosh \int_{0}^{x} P^{\frac{1}{2}} d x \cosh \int_{t}^{1} P^{\frac{1}{b}} d x
$$

approximately in general, so that

$$
\Theta^{-1} . \Phi(x, t) \text { if }\{P(t) P(x)\}^{-\frac{1}{2}} \exp \left(-\int_{x}^{t} P^{\mathfrak{k}} d x\right),
$$

which is small if $t>x$ and the real part of $P^{t}$ is positive and great along the whole path.

The statement as to the order of magnitude is not affected even when $P(0) / k^{2}$ is small, for it will be seen that the changes to be made in $\theta$ are accompanied by similar changes in $\Phi$, and the ratio of the two is not affected. Similarly when $P(1) / k^{2}$ is small. When $x$ or $t$ is near to a zero of $P$ there may be modifications in the order of magnitude of $\Phi$, but these are always in the way of reduction.

When the term $E \phi(t, x)$ predominates in $\Phi$, it is also easy to see in a general way that $\Phi / \theta$ is small under the restrictions that have been assumed.

The approximate values of $\partial \Phi / \partial x, \partial \Phi / \partial t$, and $\partial^{2} \Phi / \partial x \partial t$ differ from those of $\Phi$, when the real part of $P^{b}$ is great throughout, by the factors $\sqrt{ } P(x),-\sqrt{ } P(t),-\sqrt{ }[P(x) P(t)]$.

When the real part of $P^{3}$ is great and not of constant sign we cannot conclude that $\Phi / \theta$ is small even when $t>x$. A case of importance is when the real part of $P$ is always negative and $l i k^{2}$, while the lateral part is of variable sign and $\| k \log \log k$, in fact

$$
\eta=k \log \log k \text {. }
$$

The real part of $P^{i}$ is $\| \log \log k$, and so therefore is $\int_{0}^{1}|d \rho|$. Thus $\exp \int_{0}^{1}|d \rho|$ is at most $\|$ a finite power of $\log k$, say (log $\left.k\right)^{\delta}$, and the error is relatively small since $(\log k)^{\delta} / k$ is small. 
Also

$\{P(x) P(t)\}^{\ddagger} \Phi(x, t) / \theta$ is $\| \cosh \int_{0}^{x} d \rho \cosh \int_{t}^{1} d \rho / \sinh \int_{0}^{1} d \rho$

$$
\text { (or else } \left.\{P(0) P(1)\}^{-\frac{1}{d}} \sinh \int_{x}^{t} d \rho / \sinh \int_{0}^{1} d \rho\right) \text {, }
$$

and this cannot be more than $\| \exp \int_{0}^{1}|d \rho|$ or $(\log k)^{\delta}$.

The same method can of course be applied to $Z$ and $\Psi$; we shall write $R$ for $\lambda U-\mu V-Y$.

The same number $\delta$ will serve for both $x$ and $y$ if it is not too small.

Distribution of the Values of $\mu$ for which $\theta=0$.

27. Let $p_{1}, p_{2}$ be the greatest and least values of $S / T, q_{1}, q_{2}$ those of $U / V$, and take four positive constants $r_{1}, r_{2}, s_{1}, s_{2}$, so that

$$
r_{1}>p_{1}>p_{2}>r_{2}>s_{1}>q_{1}>q_{2}>s_{2} \text {. }
$$

Now $\quad P=-X-\lambda S+\mu T=(\xi T-\xi S-X)+\iota(\omega T-\eta S)$,

where $\lambda=\xi+\iota \eta, \mu=\xi+\iota \omega$ as in $\S 22$, and therefore if $\xi-p_{2} \xi, \xi-p_{1} \xi$ are both positive and great, the real part of $P$ is positive and great along the straight path for $x$ from 0 to 1 : the real part of $\int_{0}^{1} P^{\frac{1}{2}} d x$ is positive and great; hence $\theta$ cannot vanish (\$26). Thus when $\lambda$ is fixed and $\theta$ vanishes, a superior limit to $\xi$ is $r_{1}(\xi+\tau)$ when $\xi$ is positive, $r_{2}(\xi+\tau)$ when $\xi$ is negative, where $\tau$ may be zero when $\xi$ is of the order of $k^{2}$, but is a positive quantity of the order of $k^{2}$ when $\xi$ falls below that order.

We also know from $\S 4$ that when $\theta=0$, $\omega$ must lie between $p_{1} \eta$ and $p_{2} \eta$. Hence when $\lambda$ has a given value and $\theta=0, \mu$ must lie in a region $C$ bounded on the right by a vertical line, and above and below by two straight lines at constant distances $p_{1} \eta, p_{2} \eta$ from the real axis; on the left $C$ is unbounded. $C$ also contains the values of $\mu$ for which $P=0$, whatever the value of $x$.

The values of $\mu$ for which $Z=0$ are similarly all to be found in a region $D$ bounded by two parallels to the real axis at distances $q_{1} \eta, q_{2} \eta$ from it, and bounded on the left by the vertical line $\xi=s_{2}(\xi-\tau)$, or wl:en $\xi$ is negative $\xi=s_{1}(\xi-\tau)$;. $D$ contains also the values of $\mu$ for which $R$ vanishes.

We shall also speak of regions $C^{\prime}, D^{\prime}$ having the same vertical boundaries as $C, D$ respectively, but bounded horizontally, $C^{\prime}$ by the lines $\omega=r_{1} \eta, \omega=r_{2} \eta$, and $D^{\prime}$ by $\omega=s_{1} \eta, \omega=s_{2} \eta$. 
When $\lambda$ is real and positive, $C, D$ are simply segments of the real axis, and overlap. When $\lambda$ is complex, $C, D$ have no common point, nor have $C^{\prime}, D^{\prime}$. When $\xi$ is negative and of the order of $k^{2}$, the vertical boundaries of $C, D$ are $\xi=r_{2} \xi, \xi=s_{1} \xi$; hence the regions are separated by a horizontal distance $\left(r_{2}-s_{1}\right) \xi$, which is of the order of $k^{2}$. In particular this holds when $\eta=0$, and $C, D$ are again segments of the real axis.

Thus generally, except when $\lambda$ is real and positive, we can make a contour include zeros of $Z$ and not of $\theta$ by making it cross $D$ once and avoid $C$.

\section{The Field of Integration.}

28. For the starting point of the field of integration, when $E_{4}, G_{4}$ do not vanish, such real positive values of $\lambda, \mu$ will be taken that $\int_{0}^{1} P^{\downarrow} d x$ and $\int_{0}^{1} R^{b} d y$ are nearly equal to odd multiples of $\frac{1}{2} \iota \pi$ : we must prove that such values exist. Now the ratio of

$$
\int_{0}^{1}(\lambda S-\mu T)^{\frac{1}{3}} d x \text { to } \int_{0}^{1}(\mu V-\lambda U)^{\frac{1}{2}} d y
$$

depends only on that of $\lambda: \mu$, and decreases continually as $\mu$ increases from $s_{1} \lambda$ to $r_{2} \lambda$ : take a fraction $p / q$, whose numerator and denominator are odd, and whose value is a mean among the values of

$$
\int_{0}^{1}(\lambda S-\mu T)^{\frac{1}{2}} d x / \int_{0}^{1}(\mu V-\lambda U)^{\frac{k}{2}} d y,
$$

when $s_{1} \lambda<\mu<r_{2} \lambda$. Then the equations

$$
\begin{aligned}
& \int_{0}^{1}(\lambda S-\mu T)^{\frac{1}{2}} d x=\frac{1}{2} k p \pi, \\
& \int_{0}^{1}(\mu V-\lambda U)^{\frac{1}{2}} d y=\frac{1}{2} k q \pi,
\end{aligned}
$$

determine uniquely first the ratio and then the separate values of $\lambda, \mu$, and if $k$ is great $\int_{0}^{1} P^{\mathfrak{l}} d x$ and $\int_{0}^{1} R^{\frac{1}{b}} d y$ will differ from $\frac{1}{2} k p \pi \iota$ and $\frac{1}{2} k q \pi \iota$ respectively by small quantities of the order of $1 / k$, since

$$
P^{b}-\imath(\lambda S-\mu T)^{b} \text { and } R^{\underline{k}}-\imath(\mu V-\lambda U)^{\underline{k}}
$$

are, throughout the range, of that order. Let $g, h$ be the values of $\lambda, \mu$ thus determined. 
We shall take $k$ to be a great odd integer, and choose $g, h$ for the initial values of $\lambda, \mu$, which are real and positive, and such that $\theta, Z$ do not vanish, as was pointed out in $\S 26$. The conditions of validity of the hyperbolic approximation ( $(7)$ are fulfilled by both $P$ and $R$.

29. Let the values of $\mu$ for which $\theta=0$ when $\lambda$ has any particular real value be $m_{0}, m_{1}, m_{2}, \ldots$ in descending order of magnitude, and let those for which $Z=0$ be $\mu_{0}, \mu_{1}, \mu_{2}, \ldots$ in ascending order. Then, when $\lambda$ has its initial value $g$, no member of either series is equal to $h$, the initial value of $\mu$. Suppose $h$ to be between $m_{a}$ and $m_{a+1}$, also between $\mu_{\beta}$. and $\mu_{\beta+1}$.

Again, when $\lambda=g$ and $\mu$ is zero or negative $\int_{0}^{1} P^{\frac{1}{2}} d x$ is purely imaginary; let $h^{\prime}$ be the lowest negative value of $\mu$ for which $\int_{0}^{1}(g S-\mu T)^{\frac{k}{2}} d x$ is equal to an odd multiple of $\frac{1}{2} k \pi$, and let this multiple be $\frac{1}{2} k r \pi$. Then the circle on $h h^{\prime}$ as diameter will be chosen for the $\mu$-path when $\lambda=g . \quad R$ is constantly positive when $\lambda=g, \mu=h^{\prime}$, and hence $\mu_{0}, \mu_{1}, \ldots$ are all greater than $h^{\prime}$; also $h^{\prime}$ cannot be equal to any member of the series $m_{0}, m_{1}, \ldots$ ( $(26)$. If we suppose $h^{\prime}$ to lie between $m_{y}$ and $m_{\gamma+1}$, the $\mu$-path will enclose $\mu_{0}, \mu_{1}, \ldots, \mu_{\beta}$, and also $m_{a+1}, m_{a+2}$, $\ldots, m_{\gamma}$, but no others from either series: it will also include the point where $R=0$, and exclude that for which $P=0$, for any values of $y, x$.

The path followed by $\lambda$ will consist of the circle on $g g^{\prime}$ as diameter where $g^{\prime}$ is a negative quantity of the order of $k^{2}$ (see $\$ 31$ below). The $\mu$-path may vary continuously with $\lambda$, but must never be crossed by any of the points where $\Theta, Z, P$, or $R$ vanishes.

Now when $\lambda$ moves away from the real axis, the regions $C^{\prime}, D^{\prime}$ do the eame, and also widen out and separate from each other, their widths and distances from the axis and each other being proportional to the lateral part of $\lambda$. Their vertical boundaries depend only on the real part of $\lambda$, and when $\lambda$ moves parallel to the real axis the two regions have a motion of translation in the same direction, but with velocities respectively $r_{1}$ and $s_{2}$ times the velocity of $\lambda$ (or $r_{2}$ and $s_{1}$ times when $\lambda$ has a negative real part). Hence, as the real part of $\lambda$ changes from $g$ to $g^{\prime}, C^{\prime}$ moves to the left faster than $D^{\prime}$ until, there is a clear space between them in the horizontal direction. Then $\lambda$ moves down again to the real axis at $g^{\prime}$, and $C^{\prime}, D^{\prime}$ also move down to the real axis, but there is a gap between them ; the process is reversed as $\lambda$ travels from $g^{\prime}$ to $g$ along the lower half of its path. 
The $\mu$-path must cross $D^{\prime}$ once, between $\mu_{\beta}$ and $\mu_{\beta+1}$, and $C^{\prime}$ twice, between $m_{a}$ and $m_{a+1}$ and also between $m_{\gamma}$ and $m_{\gamma+1}$, as shewn in the figure (Fig. 2) by the dotted line. When the two regions are well apart

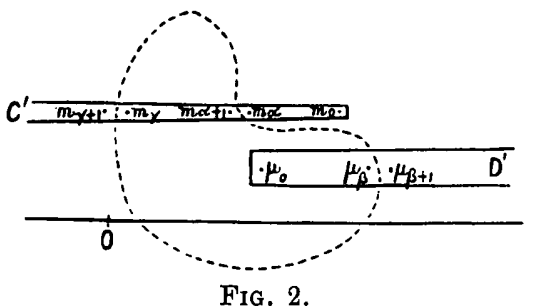

the path may be reduced to a pair of ovals, one including $\mu_{0} \ldots \mu_{\beta}$, and the other $m_{a+1} \ldots m_{\gamma}$, joined by a line which is travelled in both directions and may be left out. Then, when $\lambda$ is in the lower part of its path, the ovals may be joined again, so that the final $\mu$-path will coincide with the initial one, which it would not do without some such transition.

30. In the region of integration, if $t>x, u>y$, the subject $\Phi(x, t) \Psi(y, u) / \Theta Z$ is generally small in comparison with any negative power of $k$, but there are parts of the region where this will not always be the case, and these occur first near the initial values $g, h$, and secondly when $\mu$ is in the region $C$ or $D, \lambda$ having any value.

First, as to values near $g$, $h$; let $\eta_{1}$ denote for a moment the greater of $|\eta|$ and $|\omega|$ and let $\eta_{1}$ be $<k^{\frac{2}{2}}$. Then, since the path of $\lambda$ is circular with diameter $g g^{\prime}$, the real part of $\lambda-g$ is of no higher order than $\eta_{1}^{2} / k^{2}$, and we shall subject $\mu$ to a like restriction, so that $\xi-g, \xi-h$ will lie between $\pm e \eta_{1}^{2} / k^{2}$ where $e$ is finite and fixed. If, further, $\eta_{1}<k^{2}$, the lateral parts of $\int_{0}^{1} P^{\frac{l}{b}} d x$ and $\int_{0}^{1} R^{\frac{1}{2}} d y$ will differ from $\frac{1}{2} k p \pi \iota$ and $\frac{1}{2} k q \pi \iota$ by quantities i. $k^{-\frac{k}{2}}$. Denoting by $K$ this part of the region, where $\eta_{1}<k^{4}$, we have that $\theta$ and $Z$ do not nearly vanish within $K$.

Outside $K$, so long as $\mu$ is not in $C^{\prime}, D^{\prime}$ the real parts of $P^{k}, R^{k}$ are of constant sign and great, so that the subject of integration is small when $t>x, u>y$. It does not matter how this part of the $\mu$-path is drawn, so long as (1) the terminals are rightly placed and the condition just stated is satisfied near $g, h$; (2) $P, R$ are always $\| k^{2}$ for all values of $x, y$; (3) the length of the path is $\| k^{2}$, as will naturally follow from (2).

31. It remains to define the path of $\mu$ within $C^{\prime}$ or $D^{\prime}$, and it will in fact be necessary to vary $\lambda, \mu$ together. 
When $\xi, \eta, \omega$ have given values such that $\omega=r_{1} \eta$ (or $r_{2} \eta$ ) the lateral part of $\lambda S-\mu T$ is of constant sign, and thus the real part of $\sqrt{ }(\lambda S-\mu T)$ is of constant sign, say positive: it is increased by decreasing $\xi$ and conversely. Hence, by varying $\xi$, we can make the real part of $\int_{0}^{1} \sqrt{ }(\lambda S-\mu T) d x$ have any positive value from 0 , when $\xi$ is $+\infty$, to $\infty$, when $\xi$ is $-\infty$. We may therefore prescribe that, when $\mu$ in its path is on the upper or lower boundary of $C^{\prime}\left(\omega=r_{1} \eta\right.$ or $\left.r_{2} \eta\right)$ at the first crossing, the real part of $\int_{0}^{1} \sqrt{ }(\lambda S-\mu T) d x$ shall be equal to $\frac{1}{2} k p \pi^{*}$ as at the starting point $\lambda=g$, $\mu=h$ : in the passage between these two points we shall make $\lambda$ vary as well as $\mu$, keeping $\xi$ constant and the real part of $\int_{0}^{1} \sqrt{ }(\lambda S-\mu T) d x$ equal to $\frac{1}{2} k p \pi$, but varying $\eta, \omega$ as follows.

If $\eta \ngtr \theta k$, where $\theta$ is $\log \log k$, let $\eta$ be kept constant and $\omega$ increased continuously from $r_{2} \eta$ to $r_{1} \eta$. If $\eta>\theta k$, then diminish $\omega, \eta$ together, so that $\omega / \eta$ keeps the constant value $r_{2}$ until $\eta=\theta k$; then keep $\eta$ constant and increase $\omega$ from $r_{2} \eta$ to $r_{1} \eta$ (this is the actual crossing), and then increase $\omega, \eta$ together, keeping $\omega / \eta$ constantly $=r_{1}$ till the original value of $\lambda$ is regained.

We have proved that $\xi$ can be so chosen that the real part of $\int_{0}^{1} \sqrt{ }(\lambda S-\mu T) d x$ shall have the value $\frac{1}{2} k p \pi$ when $\omega=r_{1} \eta$ or $r_{2} \eta$, but the proof does not apply in the actual crossing when $r_{1} \eta>\omega>r_{2} \eta$. However, as long as $r_{1} \xi-\xi, r_{2} \xi-\xi$ are both positive and ' $k^{2}$, the real part of $\lambda S-\mu T$ is positive and of the same order, and, since $\eta, \omega$ are now only $\| \theta k$, the real part of $\int_{0}^{1} \sqrt{ }(\lambda S-\mu T) d x$ differs from $\int_{0}^{1} \sqrt{ }(\xi S-\xi T) d x$ by a small quantity $\| \theta^{2} / k$ ( $\S 7$, note) : thus as a first approximation we give $\xi$ such a value that $\int_{0}^{1} \sqrt{ }(\xi S-\xi T) d x=\frac{1}{2} k p \pi$, and a change : $\theta^{2}$ in $\zeta$ will give the true solution, provided always that we do not make $r_{1} \bar{\xi}-\bar{\zeta}$ or $r_{2} \xi-\xi$ negative. Now the greatest value of $\bar{\xi}$ is its initial one, $g$, and we then have $\xi=h$, which is less than $r_{2} g$. If $\xi$ decreases and $\int_{0}^{1} \sqrt{ }(\xi S-\xi T) d x$ is constant, $\xi$ must decrease too, and so also must $\xi / \xi$, until $\xi$ vanishes, $\xi$ being still of the order of $k^{2}$. Then $\xi$ decreases further and $\xi$ also vanishes and becomes negative. When both are negative and decreasing

* This condition fixes two of the terminals of the path outside $C^{\prime}, D^{\prime}$, and in a way consistent with the restriction that near $g, h$, when $\eta_{1}<k^{\frac{\pi}{4}}, \xi-g$ and $\zeta-h$ shall be $\| \eta_{1}^{2} / k^{2}$ : within $C^{r}, D^{\prime}$ this restriction may be ignored. The path outside $C^{\prime}, D^{\prime}$ is in three detached parts, and its other four terminals are fixed in the same manner at the second crossing of $C^{\prime}$ and the crossing of $D^{\prime}$. 
algebraically, the fraction $\xi / \xi$ must decrease, beginning at $+\infty$, and it reaches the value $r_{1}$ when

$$
\xi=-\left\{\frac{1}{2} k p \pi / \int_{0}^{1} \sqrt{ }\left(r_{1} T-S\right) d x^{2} .\right.
$$

If $g^{\prime}$ is algebraically greater than this quantity, $r_{1} \xi-\xi$ and $r_{2} \xi-\xi$ are always positive: and thus the value of $\xi$ is satisfactorily given by the prescribed condition.

There is a second crossing of $C^{\prime}$ in the opposite direction, where the process will be the same reversed and $\frac{1}{2} k r \pi$ will take the place of $\frac{1}{2} k p \pi$. Similarly for the crossing of $D^{\prime}$, where the values of $\omega$ on the upper and lower bounding lines are $s_{1} \eta, s_{2} \eta$, and $\xi$ is made to vary so that the real part of $\int_{0}^{1} \sqrt{ }(\mu V-\lambda U) d y=\frac{1}{2} k q \pi$. From each crossing there is derived an inferior limit for $g^{\prime}$ : only the highest of the three need be attended to. We have supposed $\eta$ positive, but there would he no essential change if it were negative, and, in fact, the whole region of integration will be supposed symmetrical about the real axes.

The advantages of this way of crossing $C^{\prime}, D^{\prime}$ are as follows; in the case of the first crossing of $C^{\prime}$, for example :

(1) Since the lateral part of $\int_{0}^{1} P^{\frac{1}{1}} d x$ is nearly $\frac{1}{2} k p \pi \iota$, its hyperbolic sine cannot nearly vanish.

(2) Since the real part of $P^{\natural}$ is $\| \theta$ during the crossing, the hyperbolic approximation may be used for $\phi$; and so $\theta$ cannot nearly vanish.

(3) We do not need to trace the variations of the points $\mu_{0}, \mu_{1}, \ldots$, $m_{0}, m_{1}, \ldots$ for complex values of $\lambda$ in order to make the $\mu$-path avoid these points. Difficulties that might be caused by branch points are thus evaded.

(4) $\Phi(x, t)(t>x)$ never becomes too great in comparison with $\theta$ as it sometimes would if $P^{\grave{k}}$ had a real part great, but not of constant sign, that is, if $\mu$ were in the region $C$ when $\eta$ was of the order of $k^{2}$.

(5) The results of $\$ \$ 22-25$ may be applied to $\psi$ when necessary, and $k_{1}: \log \log k$ at least.

The region of integration is now finally fixed; let it be called $Q$. The general idea is that $\lambda$ travels round a circle of great radius, and that $\mu$ describes a simple closed path varying with $\lambda$; the region so generated differs from $Q$ in that $Q$ has suffered deformation, but no essential change, for the sake of the advantages just now enumerated.

An effect of the deformation should be noted, namely, that $|\lambda|$ is brought down below the order of $k^{2}$ in certain parts of $Q$. When this happens, $\zeta$ and $|\mu|$ are not brought down, but are still of the same order 
as $k^{2}$. Hence, of $P$ and $R$, both do not at the same time fall below the order of $k^{2}$.

\section{Supplement to $§ 6 b$.}

32. To complete the discussion of $\S 6 b$, we shall subject $Q$ to a deformation as follows :-For every point $(\xi+\imath \eta, \xi+\iota \omega)$ belonging to $Q$ write $(\xi+\iota \eta \eta, \xi+\iota e \omega)$, and let $e$ be changed continuously from 1 to a very small positive value. This will bring the whoie region very near to the real axes, but without affecting that relation between $\mu$ and $\lambda$, which consists in $\mu$ lying within $C^{\prime}$ or $D^{\prime}$ or on the edge of either region. Make a further deformation by keeping $\lambda, \xi$ constant while $\mu$ crosses $C^{\prime}$ or $D^{\prime}$, that is, by abandoning the process of $\$ 31$ : the complications introduced by this process are unnecessary when $\omega, \eta$ are small, but the conditions as to the real parts of $\int_{0}^{1} \sqrt{ }(\lambda S-\mu T) d x$ and $\int_{0}^{1} \sqrt{ }(\mu V-\lambda U) d y$ are to be maintained in $C^{\prime}, D^{\prime}$ respectively.

In the whole course of the deformation the subject of integration, $\Phi \Psi / \Theta Z$, never becomes infinite, and thus the value of the integral is unaffected, while now $\lambda$ describes a path $L$ which is, in fact, a long loop between $g, g^{\prime}$, and to each point of $L$ there corresponds a certain path for $\mu$, say $M$.

Initially $M$ excludes $m_{0}, m_{1}, \ldots, m_{a}$, and for all positions of $\lambda$ within $L$ these points are separated from $m_{a+1}, m_{a+2}, \ldots$ by the line drawn across $C^{\prime}$ on which $\xi$ has such a value that

$$
\int_{0}^{1} \sqrt{ }\left(\xi S-\xi I^{\prime}\right) d x=\frac{1}{2} k p \pi
$$

and on which, therefore, $\theta$ cannot vanish. Similarly the points $m_{\gamma+1}$, $m_{\gamma+2}, \ldots$ are always separated from $m_{0}, m_{1}, \ldots, m_{\gamma}$, and $\mu_{0}, \mu_{1}, \ldots, \mu_{\beta}$ from $\mu_{\beta+1}, \mu_{\beta+2}, \ldots$. Hence, when $L$ is gradually reduced to a point there are no coincidences, whether with branching or otherwise, between the included and excluded members in either one of the series

$$
m_{0}, m_{1}, \ldots ; \mu_{0}, \mu_{1}, \ldots
$$

Thus we have only to discuss coincidences between the two series.

Now, if $\lambda$ moves along the real axis with unit velocity, the velocity of any one of the points $m_{0}, m_{1}, \ldots$ is a value of $d \mu / d \lambda$ given by the equation $\theta=0$, and is therefore a mean among the values of $S / T(\$ 5)$; the velocity of any one of the points $\mu_{0}, \mu_{1}, \ldots$ is similarly a mean among the values of $U / V$. Hence each of the points $m_{0}, m_{1}, \ldots$ moves relatively to any one of the points $\mu_{0}, \mu_{1}, \ldots$ with a velocity at least equal to 
$p_{2}-q_{1}$. If, then, $\lambda$ starts at $g$ and moves to the left, each of the points $m_{0}, m_{1}, \ldots, m_{\mathrm{a}}$ passes ultimately from right to left of each of the points $\mu_{0}, \ldots, \mu_{\beta}$, the process being complete before $\lambda$ arrives at $g^{\prime}$, since before that the regions $C, D$ have drawn clear of each other.

Let $\lambda_{r s}, \mu_{r s}$ be the values of $\lambda, \mu$ when $m_{r}=\mu_{s},{ }^{*}$ so that when $\lambda=\lambda_{r s}$ and $\mu=\mu_{r s}, \theta, Z$ both vanish; let $\Omega_{r s}$ be the corresponding value of $\Omega$. Then

$$
\iint_{Q} \frac{\Phi(x, t) \Psi(y, u)}{\Theta Z} d \mu d \lambda=-4 \pi^{2} \sum_{r=0}^{\mu} \sum_{s=0}^{\beta} \Omega_{r s} .
$$

Since $\Omega_{r s}$ is symmetrical in $x, t$ and also in $y, u$, there are three other expressions for the sum of the double series on the right. It will be our object to choose, out of the four, one in which the subject of integration is always small.

\section{Expansion of an Arbitrary Analytical Function.}

33. Suppose $f(x, y)$ to be an analytical function of $x, y$ throughout a rectangular area $H$, included in the square $0 \leqslant x \leqslant 1,0 \leqslant y \leqslant 1$, and having its sides $x=x_{0}, x=x_{1}, y=y_{0}, y=y_{1}$ parallel to those of the square. Then we have

$$
\begin{aligned}
&-4 \pi^{2} \sum_{r=0}^{a} \sum_{s=0}^{\beta} \iint_{H} \Omega\left(x, t, y, u, \lambda_{r s}, \mu_{r s}\right) f(t, u) d t d u \\
&=\iint_{Q} \iint_{H} \theta^{-1} Z^{-1} \Phi \Psi f(t, u) d t d u d \mu d \lambda,
\end{aligned}
$$

where $\Phi$ will stand for $\Phi(\hat{i}, x)$ when $t<x$ and $\Phi(x, t)$ when $t>x, \Psi$ for $\Psi(u, y)$ when $u<y$ and $\Psi(y, u)$ when $u>y$.

- The suffixes $r, s$ have a definite meaning. When $\mu=\kappa_{r}$, for insiance,

$$
E_{3} \phi(x, 0)+E_{4} \phi^{\prime}\left(x_{s}, 0\right)
$$

vanishes for exactly $r$ values of $x$ between 0 and 1 , unless $\dot{E}_{2}^{2}, E_{4}$ both vanish. If $E_{2}=E_{4}=0$, then $E=0$ also, and it is $E_{\mathrm{i}} \phi(x, 0)+E_{3} \phi^{\prime}(x, 0)$ that vanishes tor $r$ values of $x$ between 0 and 1 , as well as when $x=1$. (See Klein, Math. Annalen, Vol. xwir., pp. 410-427; Bôcher, licber die Reihenentwickelungen der Potentialtheorie, Teubner, 1894.) These things follow from the considerations that the roots of $E_{2} \phi(1,0)+E_{4} \phi^{\prime}(1,0)=0$ and of $\Theta=0$ separate each other, and that if $\mu, x$ vary in such a way that $E_{2} \phi(x, 0)+E_{4} \phi^{\prime}(x, 0)=0$, then they must ootn uacrease or

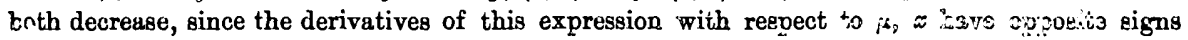
when the expression itself vanishes, for

$$
\begin{aligned}
\left\{E_{2} \phi^{\prime}(x, 0)+E_{4} \phi^{\prime \prime}(x, 0)\right\} & \frac{\partial}{\partial \mu}\left\{E_{2} \phi(x, 0)+E_{4} \phi^{\prime}(x, 0)\right\} \\
& -\left\{E_{2} \phi(x, 0)+E_{4} \phi^{\prime}(x, 0)\right\} \frac{\partial}{\partial \mu}\left\{E_{2}(x, \cdots) \cdot F^{\prime}(x, 0)\right\} \\
= & -\int_{0}^{x} T\left\{E_{9} \phi(x, 0)+E_{1} \phi^{\prime}(x, 0)\right\}^{2} a^{\prime} x_{0}
\end{aligned}
$$

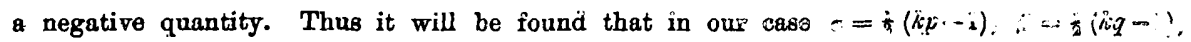
$\gamma=\frac{1}{2}(k r-1)$, and in the limit $\alpha / \beta$ has the yalue $p / q$, which ic restricted ic so ortsin finite resug of values $(\$ 28)$. 
In this equation integrate by parts on the right with respect to $t$ and again with respect to $u$. Since

$$
\begin{gathered}
\partial^{2} \Phi / \partial t^{2}=P(t) \Phi, \quad \partial^{2} \Psi / \partial u^{2}=R(u) \Psi, \\
\text { we have } \iint_{H} \Phi \Psi f(t, u) d t d u=\iint_{H} \frac{\partial \Phi}{\partial t} \frac{\partial \Psi}{\partial u} \frac{\partial^{2}}{\partial t \partial u} \frac{f(t, u)}{P(t) R(u)} d t d u \\
+\int\left\{\frac{\partial \Phi}{\partial t} \frac{\partial \Psi}{\partial u} \frac{\partial}{\partial t} \frac{f(t, u)}{P(t) R(u)} d t+\frac{\partial \Phi}{\partial t} \Psi \frac{f(t, u)}{P(t)} d u\right\},
\end{gathered}
$$

the single integral being taken along the boundary of $H$ and also along the two sides of the lines $t=x, u=y$ so far as they lie within $H$. The line $u=y$, when $y_{1}>y>y_{0}$, thus contributes

$$
-\int_{x_{0}}^{x_{1}} \frac{\partial \Phi}{\partial t} Z \frac{\partial}{\partial t} \frac{f(t, y)}{P(t) R(y)} d t
$$

since $\Psi$ has different meanings on the two sides of the line, and

$$
\frac{\partial}{\partial u}\{\Psi(u, y)-\Psi(y, u)\}=Z,
$$

when $u=y$. The contribution of the line $t=x$ is similarly, when $x_{1}>x>x_{0}$,

that is

$$
\int_{y_{v}}^{y_{1}} \theta \Psi \frac{f(x, u)}{P(x)} d u
$$

$\frac{\Theta}{P(x)}\left[\frac{\partial \Psi}{\partial u} \frac{f(x, u)}{R(u)}\right]_{y_{0}}^{y}+\frac{\Theta}{P(x)}\left[\frac{\partial \Psi}{\partial u} \frac{f(x, u)}{R(u)}\right]_{y}^{y_{1}}-\frac{\Theta}{P(x)} \int_{y_{0}}^{y_{1}} \frac{\partial \Psi}{\partial u} \frac{\partial}{\partial u} \frac{f(x, u)}{R(u)} d u$.

On account of the discontinuity in $\partial \Psi / \partial u$, when $u=y$, this expression, when $(x, y)$ lies within $H$, contains the term

$$
\Theta Z f(x, y) / P(x) R(y)
$$

besides terms at the actual limits $y_{0}, y_{1}$, and the residual integral. The contribution to the original quadruple integral on account of the same discontinuity is therefore

$$
\iint_{Q} \frac{f(x, y)}{P(x) R(y)} d \mu d \lambda
$$

in which, for the purpose of integration, $x, y$ are constants and $P(x), R(y)$ linear functions of $\lambda, \mu$. The $\mu$-path encloses the zero of $R$ and not that of $P$, while the $\lambda$-path encloses the real finite value of $\lambda$ for which these two coincide; hence the value of this contribution is, by the theory of $\S 6$,

$$
-4 \pi^{2} f(x, y) /(S V-U T) \text {. }
$$

When $(x, y)$ lies outside $H$, this term does not present itself. 
It is to be proved that the rest of the original quadruple integral tends to zero when $k$ is increased without limit: this remainder includes quadruple, triple, and double integrals, and, in general, the subjects of integration are small on account of an exponential factor of great negative index: when, however, $\lambda$ and $\mu$ are nearly real this factor becomes less important, and thus different parts of the field $Q$ have to be discussed separately.

The Remainder.-A Lemma.

34. The terms in the remainder are as follows, without regard to sign :-

(1) $\iint_{Q} \iint_{H} \frac{1}{\theta Z} \frac{\partial \Phi}{\partial t} \frac{\partial \Psi}{\partial u} \frac{\partial^{2}}{\partial t \partial u} \frac{f(t, u)}{P(t) R(u)} d t d u d \mu d \lambda$,

(2) $\iint_{Q} \int_{x_{0}}^{x_{1}} \frac{1}{\theta Z} \frac{\partial \Phi}{\partial t} \frac{\partial \Psi}{\partial u} \frac{\partial}{\partial t} \frac{f(t, u)}{P(t) R(u)} d t d \mu d \lambda \quad\left(u=y_{0}, y_{1}\right)$,

(3) $\iint_{Q} \int_{y_{0}}^{y_{1}} \frac{1}{\theta Z} \frac{\partial \Phi}{\partial t} \Psi \frac{f(t, u)}{P(t)} d u d \mu d \lambda \quad\left(t=x_{0}, x_{1}\right)$,

(t) $\iint_{Q} \frac{1}{Z P(x)} \frac{\partial \Psi}{\partial u} \frac{f(x, u)}{R(u)} d \mu d \lambda \quad\left(u=y_{0}, y_{1}\right)$,

(5) $\iint_{Q} \int_{y_{1} y_{1}}^{y_{1}} \frac{1}{Z P(x)} \frac{\partial \Psi}{\partial u} \frac{\partial}{\partial u} \frac{f(x, u)}{R(u)} d u d \mu d \lambda$,

(6) $\iint_{Q} \int_{x_{1}}^{x_{1}} \frac{1}{\Theta R(y)} \frac{\partial \Phi}{\partial t} \frac{\partial}{\partial t} \frac{f(t, y)}{P(t)} d t d \mu d \lambda$.

When $x<x_{0}$ or $>x_{1}$, the terms (4), (5) are absent, and when $y<y_{0}$ or $>y_{1},(6)$ is absent.

The absolute value of a single integral does not exceed the product of the length of the path of integration and the greatest absolute value of the subject. When we have to deal with a double integral $\iint J d \mu d \lambda$, it must be supposed that $\lambda, \mu$ are differentiable functions of two real parameters $p, g$, and that the point whose Cartesian co-ordinates are $(p, q)$ takes all positions in a certain continuum $s$ of two dimensions. The region of integration is defined by $s$ and by the relations which give $\lambda, \mu$ in terms of $p, q$, and the integral is therefore

$$
\iint_{s} J \frac{\partial(\mu, \lambda)}{\partial(p, q)} d p d q
$$

Its absolute value does not exceed the greatest absolute value of $J$ multiplied by the area of the region of integration, if we define this area as

$$
\iint_{s}\left|\frac{\partial(\mu, \lambda)}{\partial(p, q)}\right| d p d q
$$


a quantity which does not depend on the particular choice of parameters $p, q$. This area, moreover, is less than the sum of the four integrals $\iint|d \xi d \xi|, \iint|d \omega d \xi|, \iint|d \xi d \eta|, \iint|d \omega d \eta|$ taken over the same region.

In the case of the region $Q$, the range of values of each of the real variables $\xi, \eta, \xi, \omega$ is of the order of $k^{2}$, and thus the area is of the order of $k^{4}$.

35. Let us take first those parts of $Q$ in which the lateral parts of $\lambda, \mu$ are not both less than $k^{2}$. The lateral parts of $P^{k}$ and $R^{b}$ are of constant sign, since we do not enter the regions $C, D$; also, either $P$ or $R$ is constantly of the order of $k^{2}$ (\$31) and the other does not fall below that of $k^{?}$.

We have, approximately, when $t<x$, as at $\S 26$,*

$$
\Theta^{-1} \frac{\partial}{\partial t} \Phi(t, x)=\frac{1}{2}\{P(t) / P(x)\}^{\frac{1}{2}} \exp \left\{-\int_{t}^{x} P^{\underline{b}} d x\right\},
$$

and the absolute value of the exponential factor is not greater than $\exp K_{1}(t-x)$, where $K_{1}$ is the least value of the real part of $P^{\natural}$, and is therefore at least of the order of $k^{2}$.

Similarly, when $u<y$,

$$
Z^{-1} \frac{\partial}{\partial u} \Psi(u, y)=\frac{1}{2}\{R(u) / R(y)\}^{\frac{1}{2}} \exp \left\{-\int_{u}^{y} R^{\frac{1}{2}} d y\right\},
$$

where the exponential is not greater absolutely than $\exp K_{2}(u-y), K_{2}$ being also at least of the order of $k^{z}$.

Also

$$
\{P(t) R(u) / P(x) R(y)\}^{\frac{1}{2}} \frac{\partial^{2}}{\partial t \partial u} \frac{f(t, u)}{P(t) R(u)}
$$

is not of higher order than $k^{-\frac{3}{3}}$, since $f$ and its derivatives are finite, $P^{\prime}$, $R^{\prime}$ of the order of $k^{2}$, and $P R$ of that of $k^{28}$.

Again,

$$
\int_{0}^{x} e^{K_{1}(t-x)} d t<1 / K_{1}, \quad \int_{0}^{y} e^{K_{2}(u-y)} d u<1 / K_{2} .
$$

Thus in $\iiint_{y_{0}}^{y} \int_{x_{v}}^{x} \Theta^{-1} Z^{-1} \frac{\partial \Phi}{\partial t} \frac{\partial \Psi}{\partial u} \frac{\partial^{2}}{\partial t \partial u} \frac{f(t, u)}{P(t) R(u)} d t d u d \mu d \lambda$

- When the positions of $t, x$ are such that the $E$ term in $\Phi(t, x)$ is important, we ought to add to this expression for $\Theta^{-1}(\grave{o} \Phi / \partial t)$ the term

$$
E\{P(t) / P(x)\}^{\ddagger} \frac{1}{2} \exp \int_{t}^{x} P^{t} d x / E_{4}\{P(1) P(0)\}^{\frac{1}{2}} \frac{1}{2} \exp \int_{0}^{1} P^{\ddagger} d x,
$$

which here is smull as compared with any negative power of $k$, except when $t, i$ have their extreme values $(0,1)$. (Compare I., p. 98.) 
the integrations with respect to $t, u$ give a result of the order of $1 / K_{1} K_{2} k^{\text {i }}$ or $k^{-5}$, and, since the whole area of $Q$ is of the order of $k^{4}$, the quadruple integral is of the order of $1 / k$. The same holds for the other parts of $H$, that is, when $t$ runs from $x$ to $x_{1}$ or $u$ from $y$ to $y_{1}$ or both.

Hence, on this first part of $Q$, the quadruple integral (1) tends to zero when $k$ is made great.

One of the triple integrals is (6),

$$
-\iiint_{x_{0}}^{x_{1}} \Theta^{-1} \frac{\partial \Phi}{\partial t} \frac{\partial}{\partial t} \frac{f(t, y)}{P(t) R(y)} d t d \mu d \lambda,
$$

which comes out in like manner to be of the order of $k^{-\frac{1}{2}}$ at most, and the same holds for the other triple integral (5),

$$
-\iiint_{y_{0}}^{y_{1}} Z^{-1} \frac{\partial \Psi}{\partial u} \frac{\partial}{\partial u} \frac{f(x, u)}{P(x) R(u)} d u d \mu d \lambda .
$$

In the other parts (2), (3), (4) of the expression, only points on the boundary of $H$ are concerned, and the exponential factor, in $\Phi / \theta$ or $\Psi / Z$ or both, ensures that the integrals tend to the limit zero.

36. Next consider that part of $Q$ in which, while the lateral parts of $\lambda, \mu$ are both less than $k^{\mathrm{l}}$, the real parts of $P, R$ are numerically greater than $k^{22}$ for all values of $x, y$.

We may still use the hyperbolic approximation ( $(7)$, for $P, R$ do not fall to the order of $k^{a}$, and when the real part of $P^{\frac{1}{2}}$ or $R^{k}$ is not of constant sign, it is at most $\| \log \log k(\S 31)$.

The approximate value of $(\partial \Phi / \partial t) / \Theta$ contains the factor

$$
\sinh \int_{0}^{t} P^{\frac{1}{2}} d x \cosh \int_{x}^{1} P^{\frac{t}{d}} d x / \sinh \int_{0}^{1} P^{\frac{1}{t}} d x \quad(t<x)
$$

or, say, $\quad \sinh \left(u_{1}+\imath v_{1}\right) \cosh \left(u_{2}+\iota v_{2}\right) / \sinh \left(u_{3}+\iota v_{9}\right)$,

in which $\left|u_{1}\right|+\left|u_{2}\right|-\left|u_{3}\right|$ is negative unless the real part of $P^{\frac{1}{3}}$ is zero or changes sign on the $x$-path 0,1 ; only when this happens cau the factor increase indefinitely with $k$, and, since $\left|u_{1}\right|+\left|u_{2}\right|-\left|u_{3}\right|$ is at most $\therefore \log \log k$, while the argument $u_{3}+\imath v_{3}$ in the denominator is nearly $\frac{1}{2} k p \iota \pi$ or $\frac{1}{2} k r \iota \pi$, this factor and the similar factor in $(\partial \Psi / \partial u) / Z$ are at most i $(\log k)^{\delta}$ if not small $(\$ 26)$, and, in fact, only one of the two can increase indefinitely with $k$.

The other factor in the subject of integration of the quadruple integral (1) is

$$
\{P(t) R(u) / P(x) R(y)\}^{\ddagger} \frac{\partial^{2}}{\partial t \partial u} \frac{f(t, u)}{P(t) R(u)},
$$


the order of which is at most $k^{-\frac{3 e}{\sigma}}$; it remains to find the order of the area of the region of integration.

Now the range of variation is $\| k^{2}$ in the case of $\xi$ or $\xi$, and $\| k^{\frac{1}{t}}$ in that of $\omega$ or $\eta$; but, when $\xi$ is constant, the range of $\xi$ is restricted either by the conditions near $g, h(\$ 30)$ or those at the crossings of $C^{\prime}, D^{\prime}$ $(\$ 31)$, to the order of $k^{*}$, and so the order of magnitude of the area is that of $\iint|d \xi d \omega|$ or $\iint|d \eta d \xi|$, namely, $k^{2} . k^{3}$. Hence the order of this part of the quadruple integral is $(\log k)^{\delta} k^{-\frac{1}{30}}$; the same is true of the triple integrals (5), (6), (2) contributed by the lines $u=y, t=x$ and by the lines $u=y_{0}, u=y_{1}$, The terms (4) at the limits $\left(t=x, u=y_{0}, y_{1}\right)$ give a quantity $\|(\log k)^{\delta} k^{-\frac{8}{5 \pi}}$. We have left (3),

$$
\iiint_{y_{0}}^{y_{1}} \theta^{-1} Z^{-1} \frac{\partial \Phi}{\partial t} \Psi \frac{f(t, u)}{P(t)} d u d \mu d \lambda \quad\left(t=x_{0}, x_{1}\right)
$$

which, on integration by parts, gives

$$
\begin{aligned}
\iint \Theta^{-1} Z^{-1} \frac{\partial \Phi}{\partial t} \frac{\partial \Psi}{\partial u} \frac{f(t, u)}{P(t) R(u)} d \mu d \lambda \quad \text { (between limits of } u \text { ) } \\
-\iiint_{y_{0}}^{y_{1}} \Theta^{-1} Z^{-1} \frac{\partial \Phi}{\partial t} \frac{\partial \Psi}{\partial u} \frac{\partial}{\partial u} \frac{f(t, u)}{P(t) R(u)} d u d \mu d \lambda,
\end{aligned}
$$

the two terms of which are respectively $\|(\log k)^{\delta} k^{-\frac{\delta}{8}},(\log k)^{\delta} k^{-\frac{1}{\delta} \delta}$ again.

37. In what is left of the field $Q$, the crossing of one of the regions $C, D$ happens comparatively near to the vertical boundary of the other. Near the vertical boundary of $C, P$ falls below the order of $k^{2}$ for some value of $x$ and the Riccati approximation may be used for $\phi$. The real part of $P$ falls, in fact, below $k^{22^{2}}$, and generally vanishes and changes sign ; the lateral part is of constant sign, since $C$ is not entered, and of some order between $k \log \log k$ and $k^{\frac{x}{4}}$, in consequence of the provision made in $\S 31$; when $P$ has a cluster of zeros, the results of $\S \S 22-25$ are applicable, since $k_{1} \| \log \log k$ at least.

$R$, on the other hand, at the crossing of $D$, has a real part !! $k^{2}$ for all values of $y$, and there is nothing new in the behaviour of $\psi$; so that the factor $(\partial \Psi / \partial u) / Z$ is at most $\|(\log k)^{\delta}$. Leaving it out for the present and dropping also the factor $1 / R$ or $R^{\prime} / R^{2}$, which $k^{-2}$, we have two types of integral to consider, namely :

in (1), (2), (6),

$$
\text { (a) } \iiint\left|\frac{1}{\theta} \frac{\partial \Phi}{\partial t} \frac{\partial}{\partial t} \frac{\Gamma}{P(t)} d t d \mu d \lambda\right|
$$

$$
\text { (b) } \iint\left|\frac{1}{\Theta} \frac{\partial \Phi}{\partial t} \frac{\Gamma}{P(t)} \partial \mu \partial \lambda\right| \quad\left(t=x, x_{0}, x_{1}\right)
$$

8EK. 2. vor. 5. No. 973 . 
in the last form ( $(36)$ of (3), and also in (4), (5) before simplification, where $\Gamma$ may be $f(t, u)$ or $\partial f / \partial u$.

Here $\theta^{-1} \partial \Phi / \partial t$ is of no higher order than the greater of

$$
\{P(t) / P(x)\}^{\frac{1}{t}} \quad \text { and } \quad k^{3}\{P(x)\}^{-\mathrm{t}} \text {. }
$$

In $(a)$ we may put

$$
\{P(t)\}^{z} \frac{\partial}{\partial t} \frac{\Gamma}{P(t)}=\frac{4}{3} \Gamma^{\frac{2}{2}} \frac{\partial}{\partial t}\left\{\frac{\Gamma}{P(t)}\right\}^{\frac{3}{2}},
$$

and, since $\Gamma$ is finite throughout, while $P(t)$ falls to the order of $\omega$, the integration in $(a)$ with respect to $t$, when $\omega>k^{\mathbf{s}}$, gives a result $\|$

$$
\{P(x)\}^{-\frac{1}{1}} \times \text { length of path of the point }\{\Gamma / P(t)\}^{-\frac{z}{t}} \text {, }
$$

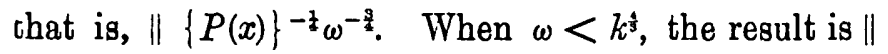

$$
k^{3}\{P(x)\}^{-\frac{1}{2}} \times \text { length of path of } \Gamma / P(t),
$$

that is, li $k^{3}\{P(x)\}^{-\frac{1}{t}} / \omega$. The type $(a)$ is thus reduced to the least favourable case of the type (b).

The further integration is with respect to $\lambda$, $\mu$, which vary as explained in $\S 31$. Let us take $\omega, \xi$ as independent variables and $\eta, \xi$ as depending on them.

As $\omega, \xi$ vary, $\eta$ is either constant $(\theta k)$ or equal to $\omega / s_{1}$ or $\omega / s_{2}$. The condition determining $\xi$ is that the real part of

$$
\int_{0}^{1} \sqrt{ }(\mu V-\lambda U) d y=\frac{1}{2} k q \pi
$$

Thus, when $\omega, \eta$ are constant, the rate of change of $\xi$ as compared with $\xi$ must be a mean among the values of $U / V$, i.e., must lie between $q_{1}$ and $q_{2}$; for, if $\mu V-\lambda U$ has its real part increased for all values of $y$, the real part of the integral will be increased and cannot be constant.

Also, when $\xi$ is constant, it is found without difficulty that $\partial \xi / \partial \omega$ \|f $\omega / k^{2}$, and is therefore small. Thus

$$
\frac{\partial(\lambda, \mu)}{\partial(\xi, \omega)}=\frac{\partial \xi}{\partial \omega}+\iota+\iota \frac{\partial(\eta, \xi)}{\partial(\xi, \omega)}-\frac{\partial \eta}{\partial \xi}=\frac{\partial \xi}{\partial \omega}+\imath-\iota \frac{\partial \xi}{\partial \xi} \frac{\partial \eta}{\partial \omega} \quad\left(\operatorname{since} \frac{\partial \eta}{\partial \xi}=0\right)
$$

and is a finita quantity, for the first term is sinall and the last lies between $-\iota q_{1} / s_{2}$ and $-\iota q_{2} / s_{1}$ when it is not zero.

Also

$$
P=-\lambda S+\mu T-X,
$$

and thus

$$
\frac{\partial P}{\partial \dot{\xi}}=-S+T \frac{\partial \xi}{\partial \xi}
$$

wirith lies between the negative finite values $q_{1} T-S$ and $q_{2} T-S$, and chiciot approach zero. 
Hence, over the part of the field where $\omega>k^{3}$, the integrals $(a),(b)$ are at most $\|$

$$
\iint\left|P^{-3} \omega^{-\frac{1}{2}} d \xi d \omega\right|
$$

which \|

$$
\iint\left|P^{-\frac{1}{2}} \frac{\partial P}{\partial \xi} \omega^{-7} d \xi d \omega\right|
$$

which $\|$ length of path of $P^{q}$, when $\omega$ is constant $\times$ length of path of $\omega^{\ddagger}$.

Now $P$ has a range of values that $! k^{2}$, and $\omega$ ranges from $k^{3}$ to $k^{2}$. Thus the integrals $(a),(b)$ over this part of the field are at most $\|_{i} k^{\frac{2}{2}} . k^{\frac{1}{20}}$, that is, $k^{\text {in }}$.

Over the part of the field where $\omega<k^{3}$, we have the subject changed by a factor $k^{\frac{1}{b}} \omega^{-\frac{1}{2}}$, and thus the integrals are \| length of path of $P^{\text {? }} \times$ length of path of $\log \omega . k^{\mathrm{s}}$, that is, \|

$$
k^{3} \cdot \log k \cdot k^{3} \quad \text { or } \quad k^{3} \log k \text {. }
$$

In each case the result is small when multiplied by the omitted factor $\|(\log k)^{\delta} / k^{2}$.

The same results follow when it is $R$ instead of $P$ that falls below the order of $k^{2}$.

Hence the whole remainder tends to zero when $k$ is increased without limit.

\section{Statement of the Result.}

38. Thus, writing $F(x, y)$ for $f(x, y) /(S V-T U)$ we have

$$
\begin{array}{r}
F(x, y)=\operatorname{Lim}_{k=\infty} \sum_{r=0}^{a} \sum_{s=0}^{\beta} \iint_{H} \Omega\left(x, t, y, u, \lambda_{r s}, \mu_{r s}\right)[S(t) V(u)-T(t) U(u)] \\
\times F(t, u) d t d u .
\end{array}
$$

Each term in the double summation is the sum of four parts, each of the form $\phi(x) \psi(y)$ multiplied by a constant (see $\S 6 a$ ), and $F(x, y)$ is therefore expanded in a series of such terms. $\lambda_{r s}, \mu_{r s}$ are such values of $\lambda, \mu$ that $\theta, Z$ both vanish ; the meaning of the suffixes $r, s$ was discussed in $\$ 32 n$. a, $\beta$ tend to infinity in a ratio that lies between certain limits: the function $F$ can be differentiated as to $x$ and to $y$ successively at least once : the expansion holds good for any point $(x, y)$ within the rectangular area $H$, over which the integration takes place; if $(x, y)$ lies outside $H$ the sum of the doubly infinite series is zero.

The theorem can easily be extended, by addition, to areas that lie 
within the unit square and are bounded wholly by lines parallel to its sides, and to functions which have lines of discontinuity parallel to those sides.

Case when $E_{4}$ or $G_{4}=0$.

39. Hitherto it has been assumed that $E_{4} \neq 0, G_{4} \neq 0$, but only a few changes in detail are needed when $E_{4}=0$ or $G_{4}=0$.

When $E_{4}, E_{2}, E_{3}, E$ are all zero, $\theta, \Phi$ reduce to single terms, and the work is exactly the same as when $E_{4} \neq 0$. The factor $\{P(0) P(1)\}$ is replaced by its reciprocal, but as it is common to the approximate values of $\theta, \Phi$, this makes no practical difference.

When $E_{2}, E_{3}$ do not both vanish it is important to notice that they cannot have the same sign, since $E_{1} E_{4}-E_{2} E_{9}-E^{2}$ must be positive and now $E_{4}=0$. The predominant terms $(\$ 26)$ in $\theta$ are apparently

$$
E_{2} \phi^{\prime}(1,0)+E_{3} \phi^{\prime}(1,0),
$$

but this may conceivably not be true if there is a terminal short section, or if

$$
E_{2}\{P(1) / P(0)\}^{\frac{1}{2}}-E_{3}\{P(0) / P(1)\}^{\frac{1}{k}}
$$

is small, in which latter case the amplitude of the complex quantity $P$ must increase by nearly an odd multiple of $2 \iota \pi$ as $x$ travels from 0 to 1 . This is impossible if either the real or the lateral part of $P$ keeps a constant sign, which is always the case in the above work.

In the case of a terminal short section there is no fresh feature if either $E_{2}$ or $E_{3}=0$, but if this is not so we must examine the possibility of the two terms destroying each other. If there is one short section, say at the end 1 , the factors $P(1)^{ \pm \ddagger}$ are to be replaced by $k^{ \pm 1}$, and this makes the $E_{3}$ term predominate, unless the factor $\chi\left(w_{n+1}\right)$ occurs and is small, and in fact if $k^{-\frac{3}{3}}$ at most. This does not happen, for $k_{1}$ is great and $\chi\left(w_{n+1}\right)$ is at least $\| k_{1} k^{-3}$.

If there are short sections at both ends, and the neighbouring section in each case is of the $q$-kind, the formula (5) of $\S 4$ enables us to see that $\Theta$ is of its full order of magnitude, that is, that the $E_{2}, E_{3}$ terms do not destroy each other, the reasoning being the same as in $\$ 20$. When the sections neighbouring the short ones are of different kinds, suppose that near the end 1 to be of the $p$-kind : then the approximations to $\phi^{\prime}(1,0)$, $\phi^{\prime}(1,0)$ contain the factors $\chi^{\prime}\left(w_{n+1}\right), \chi\left(w_{n+1}\right)$ respectively, and one term or the other may suffer a loss through this factor being small, but only one of the two $\chi, \chi^{\prime}$ can be small at once: suppose then that $\chi$ is not small. 
From (5) in $\$ 4$ we have

$$
\begin{aligned}
\left\{E_{2} \phi^{\prime}(1,0)+E_{3} \phi^{\prime}(1,0)\right\} \phi_{1}(1,0)-\left\{E_{2} \phi_{1}^{\prime}(1,0)+E_{3} \phi_{1}^{\prime}(1,0)\right\} \phi(1,0) \\
=\int_{0}^{1}\left[E_{3} \phi(x, 1) \phi_{1}(x, 1)-E_{2} \phi(x, 0) \phi_{1}(x, 0)\right]\left(P_{1}-P\right) d x,
\end{aligned}
$$

and the reasoning of $\$ 20$ applied to this shews that

$$
E_{2} \phi^{\prime}(1,0)+E_{3} \phi^{\prime}(1,0)
$$

cannot suffer loss of order, since in the first term in the square brackets $\chi$ enters and not $\chi^{\prime}$.

If $\chi$ were small we could use similarly the equation

$$
\begin{aligned}
\left\{E_{2} \phi^{\prime}(1,0)+E_{3} \phi^{\prime}\right. & (1,0)\} \phi_{1}^{\prime \prime}(1,0)-\left\{E_{2} \phi_{1}^{\prime}(1,0)+E_{3} \phi_{1}^{\prime}(1,0)\right\} \phi^{\prime \prime}(1,0) \\
& =\int_{0}^{1}\left[E_{2} \phi^{\prime}(1, x) \phi_{1}^{\prime}(1, x)-E_{3} \phi^{\prime}(x, 0) \phi_{1}^{\prime}(x, 0)\right]\left(P_{1}-P\right) d x
\end{aligned}
$$

When, lastly, the neigbbouring section is in each case of the $p$-kind the two terms contain respectively the factors*

$$
E_{2} w_{n+1}^{\prime} \chi^{\prime}\left(w_{n+1}\right) \chi\left(w_{0}\right), \quad E_{3} u v_{0}^{\prime} \chi^{\prime}\left(w_{0}\right) \chi\left(w_{n+1}\right),
$$

their other factors being common to both, and if there is a loss of order of magnitude in $\theta$ it will be because $w_{0}, w_{n+1}$ are such that

$$
E_{2} w_{n+1}^{\prime} \chi^{\prime}\left(w_{n+1}\right) \chi\left(w_{0}\right)-E_{3} 2 v_{0}^{\prime} \chi^{\prime}\left(w_{0}\right) \chi\left(w_{n+1}\right)
$$

is comparatively small, that is, below the order of $k^{*}$. Now the equation (5) of $\$ 4$ shews that any such reduction does not exceed that due to a factor $\| k_{1} k^{-\frac{1}{3}}$ (as in the final footnote to $\$ 21$ ). This will not affect the predominance of the $E_{2}$ and $E_{3}$ terms, and moreover $\Phi$ will suffer ic similar reduction, since its approximate expression also contains the factor in question. Thus the order of magnitude of $\Phi / \theta$ is not affected.

The only other special point to be attended to when $E_{4}=0$ relates to the integer $p$ of $\$ 28$, which is better taken even, unless $E_{2}, E_{3}$ vanish also. The two fractions $E_{3} / E,-E_{2} / E$ have the same sign; if they are positive, $\frac{1}{2} p$ should be even, to make the $E_{2}, E_{3}, E$ terms in $\theta$ all of the same sign at the starting point: if they are negative, $\frac{1}{2} p$ should be odd (see I., p. 99). Similarly for $r(\$ 29)$ and when $G_{4}=0$ for $q$.

- Here $w_{0}$ and $w_{0}^{\prime}$ denote the value and derivative of the Riccati argument corresponding to $x=0$, just as $w_{n+1}, w_{n+1}^{\prime}$ are similar quantities at the other end of the path. 
Double Solutions of the Equations $\theta=0, Z=0$.

40. We have supposed so far that all the solutions of the equations $\Theta=0, Z=0$ for $\lambda, \mu$ are simple. If this is not the case the only effect is a modification in the form of the series of residues $\Sigma \Omega$.

Suppose, for instance, that the curve $\theta=0$ has a node, and that $Z=0$ passes once through this node. From the conditions for a node found in $\$ 5$ it follows that $\Phi=0$ there also for all values of $x, t$ (see I., p. 89). If the $\mu$-path encloses the zero of $Z$ but not those of $\theta$, the residue in the $\mu$-integration is

$$
\Phi \Psi / \Theta \frac{\partial Z}{\partial \mu} \text {. }
$$

In the $\lambda$-integration we are to treat $\mu$ as a function of $\lambda$ defined by the relation $Z=0$, and thus at the point in question the numerator $\Phi \Psi$ has a simple zero and the denominator a double one. The contribution to the value of the double integral comes out to be

$$
-4 \pi^{2} \Psi \frac{\partial(\Phi, Z)}{\partial(\lambda, \mu)} / \frac{1}{2}\left[\frac{\partial^{2} \Theta}{\partial \lambda^{2}}\left(\frac{\partial Z}{\partial \mu}\right)^{2}-2 \frac{\partial^{2} \Theta}{\partial \lambda \partial \mu} \frac{\partial Z}{\partial \lambda} \frac{\partial Z}{\partial \mu}+\frac{\partial^{2} \Theta}{\partial \mu^{2}}\left(\frac{\partial Z}{\partial \lambda}\right)^{2}\right]
$$

if the point is enclosed once by the $\lambda$-path. By the formulæ of $\S 5$, the denominator here

$$
=-\frac{1}{2} E \int_{0}^{1} \int_{0}^{1}\left[\phi\left(x_{1}, x\right)\right]^{2}\left\{S \frac{\partial Z}{\partial \mu}+T \frac{\partial Z}{\partial \lambda}\right\}\left\{S_{1} \frac{\partial Z}{\partial \mu}+T_{1} \frac{\partial Z}{\partial \lambda}\right\} d x_{1} d x,
$$

and since

$$
\delta Z=\int_{0}^{1} \Psi(y, y)(U \delta \lambda-V \delta \mu) d y
$$

the denominator

$$
=-\frac{1}{2} E \int_{0}^{1} \int_{0}^{1} \int_{0}^{1} \int_{0}^{1}\left[\phi\left(x_{1}, x\right)\right]^{2} \Psi(y, y) \Psi\left(y_{1}, y_{1}\right)(S V-T U)\left(S_{1} V_{1}-T_{1} U_{1}\right)
$$

The factor $\frac{\partial(\Phi, Z)}{\partial(\lambda, \mu)}$ reduces similarly (see $\left.\$ 6 n\right)$ to

$$
\times d x_{1} d x d y_{1} d y \text {. }
$$

$$
-E \int_{0}^{1} \int_{0}^{1} \phi\left(x_{1}, x\right) \phi\left(x_{1}, t\right) \Psi\left(y_{1}, y_{1}\right)\left(S_{1} V_{1}-T_{1} U_{1}\right) d x_{1} d y_{1}
$$

and so we have two terms of the series $\Sigma \Omega$ replaced by

$$
\frac{\Psi(y, u) \int_{0}^{1} \int_{0}^{1} \phi\left(x_{1}, x\right) \phi\left(x_{1}, t\right) \Psi\left(y_{1}, y_{1}\right)\left(S_{1} V_{1}-T_{1} \bar{V}_{1}\right) d x_{1} d y_{1}}{\frac{1}{2} \int_{0}^{1} \int_{0}^{1} \int_{0}^{1} \int_{0}^{1}\left[\phi\left(x_{1}, x\right)\right]^{2} \Psi(y, y) \Psi\left(y_{1}, y_{1}\right)(S V-T U)\left(S_{1} V_{1}-T_{1} U_{1}\right) d x_{1} \tilde{a} ; x \tilde{a} y_{1} a y^{\prime}} .
$$


The expression in the case when $Z=0$ has a node which lies on $\theta=0$ is of the same nature.

When the curves have a common node, and the $\mu$-path encloses the zeros of $Z$ and not those of $\theta$, the value of $\lambda$ at the node being enclosed by the $\lambda$-path, the expression is found, after some calculation, to be

$$
\frac{(2 \iota \pi)^{2}\left[2 a_{1} a_{2}\left(H_{2} B_{1}-H_{1} B_{2}\right)+\left(a_{1} b_{3}+a_{2} b_{1}\right)\left(A_{1} B_{2}-A_{2} B_{1}\right)+2 b_{1} b_{2}\left(H_{1} A_{2}-H_{2} A_{1}\right)\right]}{\frac{1}{4}\left(A_{1} B_{2}+A_{2} B_{1}-2 H_{1} H_{2}\right)^{2}-\left(A_{1} B_{1}-H_{1}^{2}\right)\left(A_{2} B_{2}-H_{2}^{2}\right)}
$$

where $a_{1}, b_{1}$ are the first derivatives of $\Phi, a_{2}, b_{2}$ those of $\Psi, A_{1}, H_{1}, B_{1}$ the second derivatives of $\Theta$, and $A_{2}, H_{2}, B_{2}$ those of $Z$ with respect to $\lambda, \mu$. On substitution of the values given in $\S \S 5,6$, the numerator becomes the product of $(2 \iota \pi)^{2} E G$, and

$$
\begin{aligned}
\int_{0}^{1} \int_{0}^{1} \phi\left(x_{1}, x\right) \phi\left(x_{1}, t\right) \psi\left(y_{1}, y\right) \psi\left(y_{1}, u\right)\left[-2 S_{1} U_{1}\left(H_{2} B_{1}-H_{1} B_{2}\right)\right. \\
\left.+\left(S_{1} V_{1}+T_{1} U_{1}\right)\left(A_{1} B_{2}-A_{2} B_{1}\right)-2 T_{1} V_{1}\left(H_{1} A_{2}-H_{2} A_{1}\right)\right] d y_{1} d x_{1} .
\end{aligned}
$$

The expression in square brackets

$$
\begin{aligned}
=E G \int_{0}^{1} \int_{0}^{1} \int_{0}^{1} \int_{0}^{1}\left\{\phi\left(x_{2}, x_{9}\right)\right. & \left.\psi\left(y_{2}, y_{3}\right)\right\}^{2}\left[-2 S_{1} U_{1}\left(-U_{2} V_{3} \cdot T_{2} T_{3}+V_{2} V_{3} \cdot S_{2} T_{9}\right)\right. \\
& +\left(S_{1} V_{1}+T_{1} U_{1}\right)\left(S_{2} S_{3} V_{2} V_{3}-U_{2} U_{3} T_{2} T_{3}\right) \\
& \left.+2 T_{1} V_{1}\left(S_{3} T_{2} \cdot U_{2} U_{3}-U_{3} V_{2} S_{2} S_{3}\right)\right] d x_{3} d x_{2} d y_{3} d y_{2}
\end{aligned}
$$

which is not affected by our adding to the factor in square brackets the terms

$$
\left(S_{1} V_{1}+T_{1} U_{1}\right)\left(S_{2} T_{3} U_{3} V_{2}-S_{3} T_{2} U_{2} V_{3}\right),
$$

which change that factor into

$$
\begin{aligned}
2\left(S_{2} V_{2}-T_{2} U_{2}\right)\left(S_{1} V_{3}-T_{1}\right. & \left.U_{3}\right)\left(S_{3} V_{1}-T_{3} U_{1}\right) \\
& -\left(S_{2} V_{2}-T_{2} U_{2}\right)\left(S_{1} V_{1}-T_{1} U_{1}\right)\left(S_{3} V_{3}-T_{3} U_{3}\right) .
\end{aligned}
$$

Thus by an interchange of suffixes in the first part of this expression, we get the numerator in the form

$$
\begin{aligned}
&(2 \iota \pi E G)^{2} \int_{0}^{1} \int_{0}^{1} \int_{0}^{1} \int_{0}^{1} \int_{0}^{1} \int_{0}^{1}\left(S_{1} V_{1}-T_{1} U_{1}\right)\left(S_{2} V_{2}-T_{2} U_{2}\right)\left(S_{3} V_{3}-T_{3} U_{9}\right) \\
& \\
& \quad \times \psi\left(y_{1}, y\right) \psi\left(y_{1}, u\right)\left[\psi\left(y_{2}, y_{9}\right)\right]^{2} \\
& \\
& \quad \times\left\{2 \phi\left(x_{9}, x\right) \phi\left(x_{3}, t\right)\left[\phi\left(x_{2}, x_{1}\right)\right]^{2}-\phi\left(x_{1}, x\right) \phi\left(x_{1}, t\right)\left[\phi\left(x_{2}, x_{9}\right)\right]^{2}\right\} \\
& \times d x_{3} d x_{2} d x_{1} d y_{3} d y_{2} d y_{1} .
\end{aligned}
$$


For the factors depending on $\phi, \psi$ in the subject we may put $\frac{1}{3}\left[\Sigma \psi\left(y_{1}, y\right) \psi\left(y_{1}, u\right) \phi\left(x_{3}, x\right) \phi\left(x_{3}, t\right)\left\{\psi\left(y_{2}, y_{3}\right) \phi\left(x_{1}, x_{2}\right)\right\}^{2}\right.$

$$
\left.-\Sigma \psi\left(y_{1}, y\right) \psi\left(y_{1}, u\right) \phi\left(x_{1}, x\right) \phi\left(x_{1}, t\right)\left\{\psi\left(y_{2}, y_{3}\right) \phi\left(x_{2}, x_{3}\right)\right\}^{2}\right],
$$

the summations referring to permutations of the suffixes $1,2,3$; this expression is found to be

$$
\frac{1}{6}\{\Delta(x, y) \Delta(t, u)+\Delta(x, u) \Delta(t, y)\},
$$

where $\Delta(x, y)$ is the determinant whose first row is

$$
\phi_{1} x \psi_{1} y, \phi_{1} x \psi_{2} y, \phi_{2} x \psi_{1} y, \phi_{2} x \psi_{2} y,
$$

and whose second, third, fourth rows are formed by putting the suftixes $1,2,3$ to $x$ and $y$ in the first, where $\phi_{1}, \phi_{2}$ are two functions satisfying the equation (1), and such that $\phi_{1} \phi_{2}^{\prime}-\phi_{2} \phi_{1}^{\prime}=1 ; \psi_{1}, \psi_{2}$ are similar functions with regard to (2).

The denominator is equal to

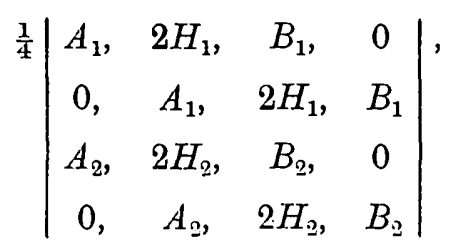

which is the product of $\frac{1}{4} E^{2} G^{2}$ and

$$
\begin{aligned}
& \int_{0}^{1} \int_{0}^{1} \int_{0}^{1} \int_{0}^{1} \int_{0}^{1} \int_{0}^{1} \int_{0}^{1} \int_{0}^{1}\left\{\phi\left(x_{1}, x\right) \phi\left(x_{2}, x_{3}\right) \psi\left(y_{1}, y\right) \psi\left(y_{2}, y_{9}\right)\right\}^{2} \\
& \times \times \begin{array}{cccc}
S S_{1}, & S T_{1}+S_{1} T, & T T_{1}, & 0 \\
0, & S_{2} S_{3}, & S_{2} T_{3}+S_{9} T_{2}, & T_{2} T_{3} \\
U U_{1}, & U V_{1}+U_{1} V, & V V_{1}, & 0 \\
0, & U_{2} U_{3}, & U_{2} V_{3}+U_{3} V_{2}, & V_{2} V_{3}
\end{array} \mid \Pi(d x d y) .
\end{aligned}
$$

The determinant differs from

$$
\begin{aligned}
2\left(S_{1} V_{8}-U_{3} T_{1}\right)\left(S_{3} V_{1}-U_{1} T_{3}\right)(S V-U T)\left(S_{2} V_{2}-U_{2} T_{2}\right) \\
-(S V-U T)\left(S_{1} V_{1}-U_{1} T_{1}\right)\left(S_{2} V_{2}-U_{2} T_{2}\right)\left(S_{3} V_{3}-U_{3} T_{9} .\right.
\end{aligned}
$$

by terms which contribute nothing to the result, since they can be made to cancel by allowable permutations of the suffixes, and the whole expression then reduces to the eightfold integral of

$$
\frac{1}{12} E^{2} G^{2}[\Delta(x, y)]^{2}(S V-T U)\left(S_{1} V_{1}-T_{1} U_{1}\right)\left(S_{2} V_{2}-T_{2} U_{2}\right)\left(S_{3} V_{3}-T_{3} U_{3}\right),
$$


by the same process as was applied to the numerator. Thus we derive denominator from numerator by putting $t=x, u=y$, multiplying by $-(S V-T U) / 16 \pi^{2}$ and integrating with respect to $x$ and $y$ from 0 to 1 .

Form of the General Term in the Expansion.

41. The equation $\theta=0$ may be understood as the condition that the multipliers of the linear transformation

$$
\left(\begin{array}{rr}
-E_{3}, & -E_{4} \\
E_{1}, & E_{2}
\end{array} \mid \begin{array}{ll}
-\phi^{\prime}(1,0), & \phi(1,0) \\
-\phi^{\prime \prime}(1,0), & \phi^{\prime}(1,0)
\end{array}\right),
$$

in which the right hand factor is $W(1,0)$, shall be the roots of

$$
\theta^{2}-2 E \theta+E_{1} E_{4}-E_{2} E_{3}=0,
$$

that is, fixed complex quantities, say $\theta_{1}, \theta_{2}$.

If we put

$$
\phi(x)=A \phi(x, 0)+B \phi^{\prime}(x, 0),
$$

we have

$$
\left\{\phi(1), \phi^{\prime}(1)\right\}=W(1,0)\left\{\phi(0), \phi^{\prime}(0)\right\},
$$

and thus it is possible to choose $A_{1}, B_{1}$ values of $A, B$, such that if $\phi_{1}$ is the corresponding function $\phi$,

$$
\begin{aligned}
-E_{3} \phi_{1}(1)-E_{4} \phi_{1}^{\prime}(1) & =\theta_{1} \phi_{1}(0), \\
E_{1} \phi_{1}(1)+E_{2} \phi_{1}^{\prime}(1) & =\theta_{1} \phi_{1}^{\prime}(0) .
\end{aligned}
$$

If $A_{2}, B_{2}, \phi_{2}$ are the complex quantities conjugate to $A_{1}, B_{1}, \phi_{1}$, then similar equations hold with $\phi_{2}, \theta_{2}$ in the place of $\phi_{1}, \theta_{1}$.

It is readily verified that $\phi_{1}(x)$ can only differ by a constant factor from

$$
\begin{array}{ll} 
& E_{3} \phi(1, x)+E_{4} \phi^{\prime}(1, x)-\theta_{1} \phi(x, 0) \\
\text { or } & E_{1} \phi(1, x)+E_{2} \phi^{\prime}(1, x)+\theta_{1} \phi^{\prime}(x, 0) \\
\text { or } & E_{1} \phi(x, 0)+E_{8} \phi^{\prime}(x, 0)+\theta_{2} \phi^{\prime}(x, 1) \\
\text { or } & E_{2} \phi(x, 0)+E_{4} \phi^{\prime}(x, 0)-\theta_{2} \phi(x, 1) .
\end{array}
$$

The constant factors in the first two cases are

and thus

$$
\left\{E_{4}-\theta_{1} \phi(1,0)\right\} / \phi_{1} 1, \quad\left\{E_{2}+\theta_{1} \phi^{\prime}(1,0)\right\} / \phi_{1} 1,
$$

$$
\begin{aligned}
\Phi(x, t) \phi_{1} 1 & =\left\{E_{2} \phi_{1} t+\theta_{1} \phi^{\prime}(1,0) \phi_{1} t-\theta_{1} \phi^{\prime}(t, 0) \phi_{1} 1\right\} \phi(x, 0) \\
& +\left\{E_{4} \phi_{1} t-\theta_{1} \phi(1,0) \phi_{1} t+\theta_{1} \phi(t, 0) \phi_{1} 1\right\} \phi^{\prime}(x, 0)+E \phi(t, x) \phi_{1} 1 \\
& =\phi_{1} t\left\{E_{2} \phi(x, 0)+E_{4} \phi^{\prime}(x, 0)-\theta_{1} \phi(x, 1)\right\}+\left(E-\theta_{1}\right) \phi(t, x) \phi_{1} 1,
\end{aligned}
$$


after some reduction. Thus

$\Phi(x, t) \phi_{1} 1 \phi_{2} 1=\phi_{1} t \phi_{2} x\left\{E_{2} \phi(1,0)+E_{4} \phi^{\prime}(1,0)\right\}+\left(E-\theta_{1}\right) \phi(t, x) \phi_{1} 1 \phi_{2} 1$, and by proper choice of the arbitrary constants in $\phi_{1}, \phi_{2}$,

$$
\Phi(x, t)=\phi_{1} t \phi_{2} x+\phi_{2} t \phi_{1} x,
$$

since the same equation holds with the interchange of the suffixes 1,2 , and

$$
E-\theta_{1}=-\left(E-\theta_{2}\right) \text {. }
$$

Also

$$
\frac{\partial \Theta}{\partial \lambda}=-\int_{0}^{1} \Phi(x, x) S d x=-2 \int_{0}^{1} \phi_{1} x \phi_{2} x S d x,
$$

and so for the other derivatives.

Hence the typical term in the expansion of $F(x, y)$ consists of four parts, which are respectively multiples of

$$
\phi_{1} x \psi_{1} y, \quad \phi_{1} x \psi_{2} y, \quad \phi_{2} x \psi_{1} y, \quad \phi_{2} x \psi_{2} y,
$$

and the coefficient of $\phi_{r} x \psi_{s} y(r, s=1,2)$ is*

$$
\begin{aligned}
\frac{1}{4} \int_{0}^{1} \int_{0}^{1} F(x, y) \phi_{3-r} x \psi_{3-s} y(S V-T U) d y d x \\
\qquad / \int_{0}^{1} \int_{0}^{1} \phi_{1} x \phi_{2} x \psi_{1} y \psi_{2} y(S V-T U) d y d x .
\end{aligned}
$$

The expression is simplified when $E_{1} E_{4}-E_{2} E_{3}-E^{2}=0$, for then $\theta_{1}=\theta_{2}=E$, and $\phi_{1}, \phi_{2}$ are the same function; the typical term is then the sum of two parts instead of four: when $G_{1} G_{4}-G_{2} G_{3}-G^{2}=0$ also, there is further simplification, the coefficient of $\phi_{1} x \psi_{1} y$ being

$$
\int_{0}^{1} \int_{0}^{1} F(x, y) \phi_{1} x \psi_{1} y(S V-T U) d y d x / \int_{0}^{1} \int_{0}^{1}\left(\phi_{1} x \psi_{1} y\right)^{2}(S V-T U) d y d x
$$

the familiar form.

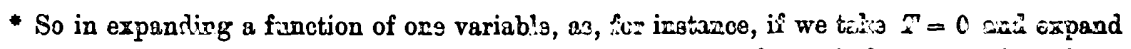

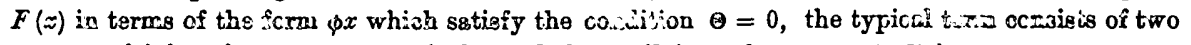
parto, multiples of $\phi_{1} x, \phi_{2} x$ respectively, and ing ccoffliciont of $\phi_{r} x(r=1,2)$ is

$$
\frac{1}{2} \int_{0}^{1} S F(x) \phi_{3-r} x d x / \int_{0}^{1} S \phi_{1} x \phi_{3} x d x \text {. (See I.) }
$$

It is ourious that the expansion oo given of a fasction that could be expressed in the form $A_{1} \phi_{1} x+A_{2} \phi_{2} x$ never coincides wici this form unless $E_{1} E_{4}-E_{2} E_{3}-E^{2}=0$. 
42. In the special case when the curve $Z=0$ passes through a node on $\theta=0$, it follows from the results of $\S 5$ that the linear transformation

$$
\left|\begin{array}{rr}
-E_{9}, & \left.-E_{4}\right) \\
E_{1}, & E_{2}
\end{array}\right| W(1,0)
$$

amounts to a simple multiplication by $E$, so that the relations

$$
\begin{aligned}
-E_{3} \phi(1)-E_{4} \phi^{\prime}(1) & =E \phi(0), \\
E_{1} \phi(1)+E_{2} \phi^{\prime}(1) & =E \phi^{\prime}(0)
\end{aligned}
$$

hold for all functions $\phi$ that satisfy (1). Let $\phi_{1}, \phi_{2}$ be two such functions in which the arbitrary constants are so determined that

$$
\phi_{1} \phi_{2}^{\prime}-\phi_{2} \phi_{1}^{\prime}=1
$$

and

$$
\int_{0}^{1} \int_{0}^{1} \phi_{1}(x) \phi_{2}(x) \Psi(y, y)(S V-T U) d y d x=0 .
$$

Then

$$
\phi\left(x_{1}, x\right)=\phi_{1} x \phi_{2} x_{1}-\phi_{1} x_{1} \phi_{2} x
$$

and thus in the fraction (50) the denominator can be simplified, and, in fact, is equal to

$$
\int_{0}^{1} \int_{0}^{1}\left(\phi_{1} x\right)^{2} \Psi(y, y)(S V-T U) d y d x \int_{0}^{1} \int_{0}^{1}\left(\phi_{2} x\right)^{2} \Psi(y, y)(S V-T U) d y d x .
$$

The numerator can be similarly transformed and the fraction breaks up into two of the customary form, namely, ${ }^{*}$

$$
\begin{aligned}
& \Psi(y, u) \phi_{1} x \phi_{1} t / \int_{0}^{1} \int_{0}^{1}\left(\phi_{1} x\right)^{2} \Psi(y, y)(S V-T U) d y d x \\
& +\Psi(y, u) \phi_{2} x \phi_{2} t / \int_{0}^{1} \int_{0}^{1}\left(\phi_{2} x\right)^{2} \Psi(y, y)(S V-T U) d y d x .
\end{aligned}
$$

The same kind of thing happens when the curves $\theta=0, Z=0$ have a node in common: the corresponding term in $\Sigma \Omega$ has been discussed $(\S 40)$, and to simplify it we go on as follows. If $\phi_{1}, \phi_{2}$ are two inde-

* Similarly, if $F(x)$ is expanàed in multiples of functions $\phi$ (last note), when the equation $\Theta=0$ gives two equal values of $\lambda$ the expansion has the corresponding terms

$$
\phi_{1} x \int_{0}^{1} S F(x) \phi_{1} x d x / \int_{1}^{1} S\left(\phi_{1} x\right)^{2} d x+\phi_{2} x \int_{0}^{1} S F(x) \phi_{2} x d x / \int_{0}^{1} S\left(\phi_{3} r\right)^{2} d x .
$$

The trigonometrical series in sines an $\not$ cosines is, in fact, a case of this, where $S=1, X=0$, $E_{1}=E_{4}=0, E_{2}=-E_{3}=E$, so that

$$
\theta=2(\cos \sqrt{ } \lambda-1),
$$

and $\lambda=(2 n \pi)^{2}$ is a double solution of $\Theta=0$, corresponding values of $\phi_{1}, \phi_{2}$ being

$$
\cos 2 n \pi x, \quad \frac{1}{2 n \pi} \sin 2 n \pi x .
$$


pendent solutions of (1), and $\psi_{1}, \psi_{2}$ the like for (2), and

$$
(\phi \psi)_{r}=A_{r} \phi_{1} \psi_{1}+B_{r} \phi_{1} \psi_{2}+C_{r} \phi_{2} \psi_{1}+D_{r} \phi_{2} \psi_{2},
$$

then

$$
\int_{0}^{1} \int_{0}^{1}(\phi \psi)_{r}(\phi \psi)_{s}(S V-T U) d y d x
$$

is a symmetrical bilinear function of the two sets of coefficients $A, B, C, D$, say

$$
(a b c d f g h l m n)\left(A_{r} B_{r} C_{r} D_{r}\right)\left(A_{s} B_{s} C_{s} D_{s}\right),
$$

where, for instance,

$$
\begin{gathered}
a=\int_{0}^{1} \int_{0}^{1} \phi_{1}^{2} \psi_{1}^{2}(S V-T U) d y d x, \\
f=l=\int_{0}^{1} \int_{0}^{1} \phi_{1} \phi_{2} \psi_{1} \psi_{2}(S V-T U) d y d x .
\end{gathered}
$$

Another relation among the coefficients can be found, for any such expression as

$$
\int_{0}^{1} \int_{0}^{1} \int_{0}^{1} S_{1} T_{2} T_{3} \phi\left(x_{2}, x_{3}\right) \phi\left(x_{3}, x_{1}\right) \phi\left(x_{1}, x_{2}\right) d x_{3} d x_{2} d x_{1}
$$

vanishes, since its sign is reversed by at least one permutation of the suffixes $1,2,3$. Hence

$$
\begin{aligned}
\int_{0}^{1} \int_{0}^{1} \int_{0}^{1} \int_{0}^{1} \int_{0}^{1} \int_{0}^{1} I_{r=1}^{3} & \left(S_{r} V_{r}-T_{r} U_{r}\right)\left(\phi_{1} x_{2} \phi_{2} x_{3}-\phi_{2} x_{2} \phi_{1} x_{3}\right)\left(\phi_{1} x_{3} \phi_{2} x_{1}-\phi_{2} x_{3} \phi_{1} x_{1}\right) \\
& \times\left(\phi_{1} x_{1} \phi_{2} x_{2}-\phi_{2} x_{1} \phi_{1} x_{2}\right)\left(\psi_{1} y_{1} \psi_{2} y_{3}\right)^{2} \psi_{1} y_{2} \psi_{2} y_{2} \Pi d x d y=0
\end{aligned}
$$

which, on expansion, gives

$$
-a f d-c h m-b g n+b c l+g h d+a m n=0
$$

or

$$
\left|\begin{array}{ccc}
a & h & l \\
g & f & n \\
l & m & a
\end{array}\right|-\left|\begin{array}{ccc}
h & b & f \\
g & f & c \\
l & m & n
\end{array}\right|=0 .
$$

Hence the two quadrics $(a b c d f g h l m n \chi A B C D)^{2}$, and

$$
A D-B C
$$

are such that both the invariants $\theta, \theta^{\prime}$ (Salmon, Solid Geometry of Three Dimensions, Fourth Edition, p. 173) vanish. Hence four sets of quantities

$$
A_{r}, B_{r}, C_{r}, D_{r} \quad(r=1,2,3,4)
$$

can be found such that $\quad A_{r} D_{r}-B_{r} C_{r}=0$, 
1906.] Harmonic eXpansions of FUnCtions of two variarles.

and

$$
\left(a b c d f g h l m n \gamma\left(A_{r} B_{r} C_{r} D_{r}\right)\left(A_{s} B_{s} C_{s} D_{s}\right)=0 \quad(r \neq s) .\right.
$$

Now $A_{r} D_{r}-B_{r} C_{r}=0$ is the condition that $(\phi \psi)_{r}$ should be the product of factors, say $\phi_{r} \psi_{r}$, and bence four products

$$
\phi_{1} \psi_{1}, \quad \phi_{2} \psi_{2}, \quad \phi_{3} \psi_{3}, \quad \phi_{4} \dot{\psi}_{4}
$$

can be found such that

$$
\int_{0}^{1} \int_{0}^{1} \phi_{r} \psi_{r} \phi_{s} \psi_{s}(S V-T U) d y d x=0 \quad(r \neq s) .
$$

The rows in $\Delta(x, y)$ may now be taken as of the form

$$
\phi_{1} \psi_{1}, \quad \phi_{2} \psi_{2}, \phi_{3} \psi_{3}, \phi_{4} \psi_{4},
$$

and the denominator of the term in $\Sigma \Omega$ is a constant multiple of

$$
I_{r=1}^{4} \int_{0}^{1} \int_{0}^{1}(S V-T U) \phi_{r}^{2} \psi_{r}^{2} d y d x,
$$

since, when $\Delta(x, y)$ is expanded and squared, all the double product terms contribute nothing to the integral.

Treating the numerator similarly we find that the term in $\Sigma \Omega$ takes the form

$$
\sum_{r=1}^{4} \phi_{r} x \phi_{r} t \psi_{r} y \psi_{r} u / \int_{0}^{1} \int_{0}^{1}(S V-T U) \phi_{r}^{2} \psi_{r}^{2} d y d x,
$$

which is the sum of four terms of the usual type.

Hence, if the curves $\theta=0, Z=0$ meet in a point which is a node on either or both, the form of expansion is not affected, but for two or four terms in the expansion the values of $\lambda, \mu$ are the same.

Another special case, which includes most of those in which the expansions are commonly used, is when

and, of course, $\begin{aligned} E_{1} E_{4} & =E_{2} E_{9} \\ E & =0 .\end{aligned}$

Thus $\theta_{1}=\theta_{2}=0$, and the general term is a multiple of $\phi_{1} x$, a function satisfying (1) and the further conditions

$$
\begin{aligned}
& E_{1} \phi_{1}(1)+E_{2} \phi_{1}^{\prime}(1)=0, \\
& E_{1} \phi_{1}(0)+E_{3} \phi_{1}^{\prime}(0)=0,
\end{aligned}
$$

so that at each limit the ratio of the function to its derivative has a prescribed value, which may of course be zero or infinite.

43. The expansion is not generally valid at the boundary of $H$; but when that boundary coincides, wholly or in part, with the boundary of the unit square there are some special cases (compare I., pp. 101-2). 
The expansion holds when $y=0,1$, if

(1) $F(x, 1)=F(x, 0)=0$,

or (2) $G_{1}, G_{2}, G_{3}, G$ vanish,

or (3) $U, V, Y$ have the same values at the two limits and $G_{1}=0$,

$$
G_{2}=m G=-m^{2} G_{9}, F(x, 1)=m F(x, 0) .
$$

Similarly when $x=0,1$.

In case (3), taking $m=1$, we have expansions in periodic functions. The present proof does not apply to Lame's expansions on a complete ellipsoid, because in Lamés case $S V-U T$ vanishes for one pair of values of $x, y$. The expansions moreover are of different kinds, the array of terms in this paper being rectangular, whereas in Lame's formulæ it is triangular, the complete $n$-th term including $2 n+1$ partial terms. It might be possible to prove the Lamé expansions by taking a quadratic form in $\theta, Z$, instead of their simple product, as denominator in the subject of integration. 